\title{
From Communicating Machines to Graphical Choreographies
}

\author{
Julien Lange Nobuko Yoshida \\ Imperial College London, UK \\ $\{$ j.lange, n.yoshida $\}$ @imperial.ac.uk
}

\author{
Emilio Tuosto \\ University of Leicester, UK \\ emilio@le.ac.uk
}

\begin{abstract}
Graphical choreographies, or global graphs, are general multiparty session specifications featuring expressive constructs such as forking, merging, and joining for representing application-level protocols. Global graphs can be directly translated into modelling notations such as BPMN and UML. This paper presents an algorithm whereby a global graph can be constructed from asynchronous interactions represented by communicating finite-state machines (CFSMs). Our results include: a sound and complete characterisation of a subset of safe CFSMs from which global graphs can be constructed; an algorithm to translate CFSMs to global graphs; a time complexity analysis; and an implementation of our theory, as well as an experimental evaluation.
\end{abstract}

Keywords multiparty session types, choreography, communicating finite state machines, global graphs, theory of regions

\section{Introduction}

Context Choreographies, models of interactions among software components from a global point of view, have been advocated as a conceptual and practical tool to tackle the complexity of designing, analysing, and implementing modern applications (see e.g., [4, 11, 17 26]). As noted in [17], besides yielding a global perspective of the coordination of applications supporting the development and verification of single components, a global specification can also be projected so to obtain the local behaviour of components. The software engineering methodology associated with choreographies is usually a uni-directional (top-down) approach to software development life cycle (SDLC). Such a methodology appeals to industry [4, 5, 17] since it allows developers to check components against the corresponding projections of the choreography. However, choreography-based approaches do not fully support SDLC. For example, the 'conform direction' of testable architectures [4] lacks algorithms to obtain global models when modifying local projections.

To address this limitation we propose an algorithm to construct choreographies from a set of behavioural specifications of components interacting through asynchronous message passing. We consider the following two scenarios to motivate the practical applicability of our algorithm.

- Distributed service architectures envisage software as a provision made available (through a public interface that hides implementation details) to be dynamically searched by and composed. The choreography of such systems cannot therefore be designed in advance and has to be established and checked at binding-time to attain automatic composition.

- A frequent problem practitioners have to face is the integration of newly developed software with legacy code. Typically, the latter often do not come with a global specification and changes with time. Therefore, it is difficult to assess how modifications to newly developed components fit within the system.

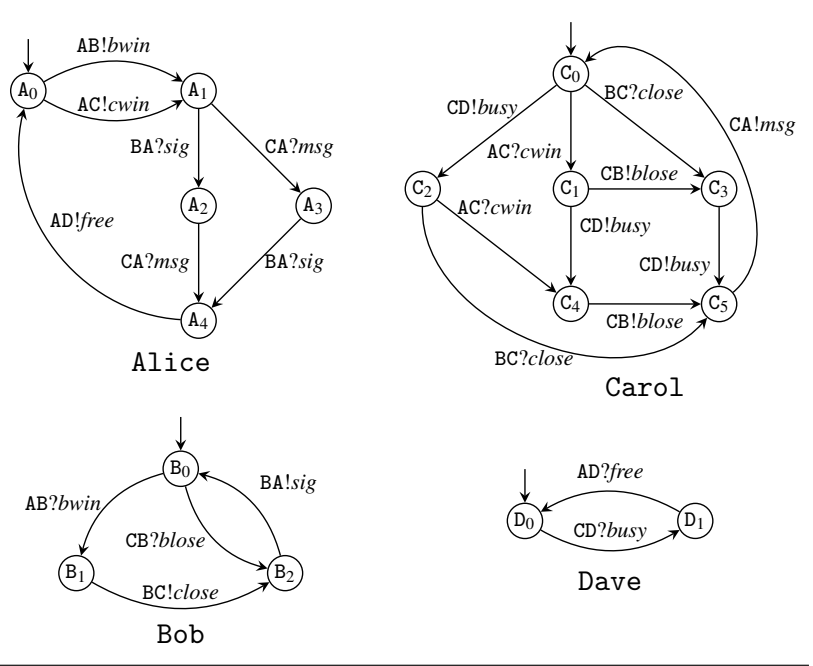

Figure 1. Communicating System $S_{r e}$

Relying on a modelling notations used in industry, our algorithm enables a bi-directional (top-down and bottom-up) choreographydriven SDLC: a developer can visualise a global viewpoint; thus, when an unexpected choreography emerges, either existing components or the global specification may be refined. Modified choreographies can be projected again so to be compared with the original projections.

Our approach We adopt communicating finite-state machines (CFSMs) as suitable behavioural specifications of distributed components from which a choreography can be built. CFSMs are a conceptually simple model, based on asynchronous FIFO messagepassing communication, and are well-established for analysing properties of distributed systems. They are also widely used in industry tools and can be seen as end-point specifications.

We define an algorithm that, given a set of CFSMs, yields a choreography expressed as a global graph [19], a graphical model closely related to BPMN 2.0 Choreography, advocated as a suitable notation for services [1]. The system $S_{r e}$ in Figure 1 will be the running example to illustrate our approach; $S_{r e}$ consists of four CFSMs, each having three buffers to communicate with the other participants, that realise a protocol of a fictive game where:

1. Alice (A) sends either bwin to Bob (B) or cwin to Carol (C) to decide who wins the game. In the former case, A fires the transition $\mathrm{AB}$ ! bwin whereby the message bwin is put in the FIFO buffer $A B$ from $A$ to $B$, and likewise in the latter case.

2. If $B$ wins (that is the message bwin is on top of the queue $A B$ and $\mathrm{B}$ consumes it by taking the transition $\mathrm{AB}$ ? bwin), then he 


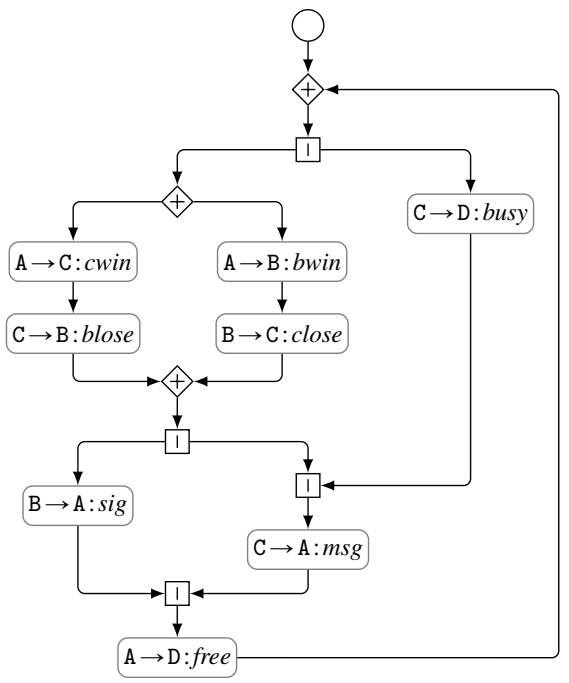

Figure 2. Global Graph $G_{r e}$

sends a notification (close) to C to notify her that she has lost. Symmetrically, C notifies B of her victory (blose).

3. During the game, C notifies Dave (D) that she is busy.

4. After $B$ and $C$ have been notified of the outcome of the game, B sends a signal ( $\mathrm{sig}$ ) to $\mathrm{A}$, while $\mathrm{C}$ sends a message ( $\mathrm{msg}$ ) to A.

5. Once the result is sent, A notifies $\mathrm{D}$ that $\mathrm{C}$ is now free and a new round starts.

The underlying protocol of $S_{r e}$ shows that CFSMs capture many coordination constructs: in 1. A (non-deterministically) chooses the winner; in 2 B has a sequential behaviour; in 3 , the parallel behaviour of $\mathrm{C}$ is rendered with the interleaving of transition CD!busy; in 4 and 5 , threads join and finally the protocol loops.

Understanding the global model of $S_{r e}$ is not easy. A much clearer specification is given by the global graph $G_{r e}$ (constructed by our algorithm) in Figure 2 There, the choreography of the four components is explicit and it is possible to identify sequentially ordered, independent, or exclusive interactions. For instance, from $G_{r e}$, it is evident that interaction $\mathrm{A} \rightarrow \mathrm{B}:$ bwin must precede $\mathrm{B} \rightarrow \mathrm{C}:$ close, while interaction $\mathrm{C} \rightarrow \mathrm{D}:$ busy is independent of the former two. On the other hand, $\mathrm{A} \rightarrow \mathrm{B}:$ bwin and $\mathrm{A} \rightarrow \mathrm{C}:$ cwin are exclusive, i.e., only one of them may be executed in each round of the game.

Establishing properties of CFSMs such as

Is $S_{r e}$ deadlock-free? will any sent message be eventually consumed? will each participant eventually receive any message s/he is waiting for?

is generally undecidable [13] or computationally hard, and not immediate even for the simple scenario in Figure 1 . We give a decidable condition, called generalised multiparty compatibility (GMC) that characterises a set of systems for which the questions above can be decided. Our algorithm can produce a global graph from any set of generalised multiparty compatible CFSMs. The global graph is constructed through a transformation of the CFSMs into a safe Petri net, using the algorithm in [18]. The transformation preserves the original CFSMs, which can be recovered by projecting the global graph. Noteworthy, most of the systems we found in the literature enjoy GMC and very few of them do not (cf. $\S 5$.

Contributions To the best of our knowledge, this is the first work to build graphical choreographies from CFSMs and to characterise the set of CFSMs from which such choreographies can be built. Our theory is supported by a tool (which we evaluated against protocols from the literature).

Recently the construction of syntactic (non-graphical) multiparty session types [23] from local specifications has been studied in [20, 24] for a less general framework with no support for local concurrency; for instance in Figure 1 C can send message busy while concurrently receiving either cwin or close (similarly A can execute input actions CA?msg and BA?sig in parallel). We argue that catering for a general form of local concurrency (which is in fact supported by threads in many programming languages) is crucial for modelling real-world systems.

In [10, 11] conditions for communicating systems to be safe are given; however, they do not address the problem of constructing choreographies and consider a form of local concurrency more restrictive than ours due to a single receiving buffer per participant. We use two uni-directional queues for each couple of participants so that a component can concurrently communicate with many other components accessing different FIFO queues (as, e.g., supported in the TCP protocol suite).

Synopsis $\S 2$ reviews CFSMs. $\S 3$ defines generalised multiparty compatibility, analyses its complexity (Proposition 3.1 and Proposition 3.2, and its soundness (Theorem 3.1. $\$ 3.3$ discusses how our condition can be used to suggest amendments to fix nonGMC systems. The construction algorithm, its complexity (Proposition 4.1), and its completeness (Theorem 4.1) are in $\$ 4$ The tool and experimental evaluation are in $\S 5$ We conclude and discuss future work in $\S 7$ after discussing more related work in $\S 6$ The full version of this paper [?] include full proofs of our results and benchmark protocols; our tool is available online [2].

\section{Communicating Finite-State Machines}

This section reviews definitions and properties of CFSMs. Throughout the paper we use the following sets and notations. Fix a finite set $\mathcal{P}$ of participants (ranged over by p, q, r, s, etc.) and a finite alphabet $\mathbb{A}$. The set of channels is $C \stackrel{\text { def }}{=}\{\mathrm{pq} \mid \mathrm{p}, \mathrm{q} \in \mathcal{P}$ and $\mathrm{p} \neq \mathrm{q}\}$ while $A c t \stackrel{\text { def }}{=} C \times\{!, ?\} \times \mathbb{A}$ is the set of actions (ranged over by $\ell$ ), $\mathbb{A}^{*}$ (resp. $A c t^{*}$, ranged over by $\varphi$ ) is the set of finite words on $\mathbb{A}$ (resp. Act). Also, $\varepsilon(\notin \mathbb{A} \cup A c t)$ is the empty word, $|\varphi|$ denotes the length of $\varphi$, and $\varphi \varphi^{\prime}$ is the concatenation of $\varphi$ and $\varphi^{\prime}$ (we overload these notations for words over $\mathbb{A}$ ).

Definition 2.1 (CFSM). A communicating finite-state machine is a finite transition system given by a 4-tuple $M=\left(Q, q_{0}, \mathbb{A}, \delta\right)$ where $Q$ is a finite set of states, $q_{0} \in Q$ is the initial state, and $\delta \subseteq Q \times A c t \times Q$ is a set of transitions.

The transitions of a CFSM are labelled by actions; label sr! $a$ represents the sending of message $a$ from machine s to $r$ and, dually, sr? $a$ represents the reception of $a$ by $\mathrm{r}$. We write $\mathcal{L}(M) \subseteq A c t^{*}$ for the language on Act accepted by the automaton corresponding to machine $M$ where each state of $M$ is an accepting state. A state $q \in Q$ with no outgoing transition is final; $q$ is a sending (resp. receiving) state if all its outgoing transitions are labelled with sending (resp. receiving) actions, and $q$ is a mixed state otherwise.

A CFSM $M=\left(Q, q_{0}, \mathbb{A}, \delta\right)$ is deterministic if for all states $q \in Q$ and all actions $\ell \in A c t$, if $\left(q, \ell, q^{\prime}\right),\left(q, \ell, q^{\prime \prime}\right) \in \delta$ then $q^{\prime}=q^{\prime \prime}{ }^{1} \mathrm{~A}$ CFSM $M$ is minimal if there is no machine $M^{\prime}$ with fewer states and transitions than $M$ such that $\mathcal{L}(M)=\mathcal{L}\left(M^{\prime}\right)$. Hereafter, we only consider deterministic and minimal CFSMs.

\footnotetext{
${ }^{1}$ Sometimes, a CFSM is considered deterministic when $\left(q, \operatorname{sr} ! a, q^{\prime}\right) \in \delta$ and $\left(q, \operatorname{sr} ! a^{\prime}, q^{\prime \prime}\right) \in \delta$ then $a=a^{\prime}$ and $q^{\prime}=q^{\prime \prime}$. Here, we follow a different definition [16] in order to represent branching type constructs.
} 
Definition 2.2 (Communicating systems). Given a CFSM $M_{\mathrm{p}}=$ $\left(Q_{\mathrm{p}}, q_{0 \mathrm{p}}, \mathbb{A}, \delta_{\mathrm{p}}\right)$ for each $\mathrm{p} \in \mathcal{P}$, the tuple $S=\left(M_{\mathrm{p}}\right)_{\mathrm{p} \in \mathcal{P}}$ is a communicating system $(C S)$. A configuration of $S$ is a pair $s=(\vec{q} ; \vec{w})$ where $\vec{q}=\left(q_{\mathrm{p}}\right)_{\mathrm{p} \in \mathcal{P}}$ with $q_{\mathrm{p}} \in Q_{\mathrm{p}}$ and where $\vec{w}=\left(w_{\mathrm{pq}}\right)_{\mathrm{pq} \in C}$ with $w_{\mathrm{pq}} \in \mathbb{A}^{*} ;$ component $\vec{q}$ is the control state and $q_{\mathrm{p}} \in Q_{\mathrm{p}}$ is the local state of machine $M_{\mathrm{p}}$. The initial configuration of $S$ is $s_{0}=\left(\overrightarrow{q_{0}} ; \vec{\varepsilon}\right)$ with $\overrightarrow{q_{0}}=\left(q_{0 \mathrm{p}}\right)_{\mathrm{p} \in \mathcal{P}}$.

Hereafter, we fix a machine $M_{\mathrm{p}}=\left(Q_{\mathrm{p}}, q_{0 \mathrm{p}}, \mathbb{A}, \delta_{\mathrm{p}}\right)$ for each participant $\mathrm{p} \in \mathcal{P}$ and let $S=\left(M_{\mathrm{p}}\right)_{\mathrm{p} \in \mathcal{P}}$ be the corresponding system.

Definition 2.3 (Reachable states and configurations). A configuration $s^{\prime}=\left(\vec{q}^{\prime} ; \vec{w}^{\prime}\right)$ is reachable from another configuration $s=(\vec{q} ; \vec{w})$ by firing transition $\ell$, written $s \stackrel{\ell}{\rightarrow} s^{\prime}$ (or $s \rightarrow s^{\prime}$ if the label is immaterial), if there is $a \in \mathbb{A}$ such that either:

1. $\ell=\mathrm{sr} ! a$ and $\left(q_{\mathrm{s}}, \ell, q_{\mathrm{s}}^{\prime}\right) \in \delta_{\mathrm{s}}$ and

(a) $q_{\mathrm{p}}^{\prime}=q_{\mathrm{p}}$ for all $\mathrm{p} \neq \mathrm{s}$, and

(b) $w_{\mathrm{sr}}^{\prime}=w_{\mathrm{sr}} \cdot a$ and $w_{\mathrm{pq}}^{\prime}=w_{\mathrm{pq}}$ for all $\mathrm{pq} \neq \mathrm{sr}$; or

2. $\ell=\operatorname{sr} ? a$ and $\left(q_{\mathrm{r}}, \ell, q_{\mathrm{r}}^{\prime}\right) \in \delta_{\mathrm{r}}$ and

(a) $q_{\mathrm{p}}^{\prime}=q_{\mathrm{p}}$ for all $\mathrm{p} \neq \mathrm{r}$, and

(b) $w_{\mathrm{sr}}=a \cdot w_{\mathrm{sr}}^{\prime}$ and $w_{\mathrm{pq}}^{\prime}=w_{\mathrm{pq}}$ for all $\mathrm{pq} \neq \mathrm{sr}$.

The reflexive and transitive closure of $\rightarrow$ is $\rightarrow^{*}$. We write $s_{1} \stackrel{\ell_{1} \cdots \ell_{m}}{\longrightarrow} s_{m+1}$ when, for some $s_{2}, \ldots, s_{m}, s_{1} \stackrel{\ell_{1}}{\longrightarrow} s_{2} \cdots s_{m} \stackrel{\ell_{m}}{\longrightarrow} s_{m+1}$. A sequence of transitions is $k$-bounded if no channel of any intermediate configuration on the sequence contains more than $k$ messages. The set of reachable configurations of $S$ is $R S(S)=\left\{s \mid s_{0} \rightarrow{ }^{*} s\right\}$. The k-reachability set of $S$ is the largest subset $R S_{k}(S)$ of $R S(S)$ within which each configuration $s$ can be reached by a $k$-bounded execution from $s_{0}$.

Condition $1 \mathrm{~b}$ in Definition 2.3 puts $a$ on channel sr, while $2 \mathrm{~b}$ gets $a$ from channel sr. Note that, for every integer $k$, the set $R S_{k}(S)$ is finite and computable.

We now recall several definitions about communicating systems $S$ and their configurations $s=(\vec{q} ; \vec{w})$. We say that $s$ is a deadlock configuration [16] Def. 12] if $\vec{w}=\vec{\varepsilon}$, there is $r \in \mathcal{P}$ such that $\left(q_{\mathrm{r}}, \mathrm{sr} ? a, q_{\mathrm{r}}^{\prime}\right) \in \delta_{\mathrm{r}}$, and for every $\mathrm{p} \in \mathcal{P}, q_{\mathrm{p}}$ is a receiving or final state, i.e., all the buffers are empty, there is at least one machine waiting for a message, and all the other machines are either in a final or receiving state. Configuration $s$ is an orphan message configuration if all $q_{\mathrm{p}} \in \vec{q}$ are final but $\vec{w} \neq \vec{\varepsilon}$, i.e., there is at least a non-empty buffer and each machine is in a final state. Finally, $s$ is an unspecified reception configuration [16. Def. 12] if there exists $r \in \mathcal{P}$ such that $q_{\mathrm{r}}$ is a receiving state, and $\left(q_{\mathrm{r}}, \mathrm{sr} ? a, q_{\mathrm{r}}^{\prime}\right) \in \delta_{\mathrm{r}}$ implies that $\left|w_{\mathrm{sr}}\right|>0$ and $w_{\mathrm{sr}} \notin a \mathbb{A}^{*}$, i.e., $q_{\mathrm{r}}$ is prevented from receiving any message from any of its buffers.

Definition 2.4 (Safe CS). System $S$ is safe if for each $s \in R S(S), s$ is not a deadlock, an orphan message, nor an unspecified reception configuration.

The following definitions are new and instrumental for $\S 3$ where we characterise a subset of safe CS from which a global graph can be constructed. A key point to give our condition for a CS to be safe is to identify sets of concurrent actions. Below, we define an equivalence relation on transitions of a CFSM. Given $q, q^{\prime} \in Q$, let $\operatorname{act}\left(q, q^{\prime}\right) \stackrel{\text { def }}{=}\left\{\ell \mid\left(q, \ell, q^{\prime}\right) \in \delta\right\}$ and define $\diamond, \bullet \subseteq \delta \times \delta$ as the smallest equivalence relations that respectively contain the relations $\unrhd$ and $\bullet$ where

$$
\begin{aligned}
- & \left(q_{1}, \ell, q_{2}\right) \triangleq\left(q_{1}^{\prime}, \ell, q_{2}^{\prime}\right) \text { iff } \ell \notin \operatorname{act}\left(q_{1}, q_{1}^{\prime}\right)=\operatorname{act}\left(q_{2}, q_{2}^{\prime}\right) \neq \varnothing \\
\cdot & \left(q_{1}, \ell, q_{2}\right) \bullet\left(q_{1}^{\prime}, \ell, q_{2}^{\prime}\right) \text { iff }\left(q_{1}, \ell, q_{2}\right) \triangleq\left(q_{1}^{\prime}, \ell, q_{2}^{\prime}\right) \text { and for all }\left(q, \ell, q^{\prime}\right) \in \\
& {\left[\left(q_{1}, \ell, q_{2}\right)\right]^{\diamond}, \operatorname{act}\left(q_{1}, q\right)=\operatorname{act}\left(q_{2}, q^{\prime}\right) \wedge \operatorname{act}\left(q_{1}^{\prime}, q\right)=\operatorname{act}\left(q_{2}^{\prime}, q^{\prime}\right) }
\end{aligned}
$$

where $\left[\left(q, \ell, q^{\prime}\right)\right]^{\diamond}$ denotes the equivalence class of $\left(q, \ell, q^{\prime}\right)$ wrt $\diamond$.
Intuitively, two transitions are -related if they refer to the same action up-to interleaving.

Example 2.1. Consider the CFSM below.

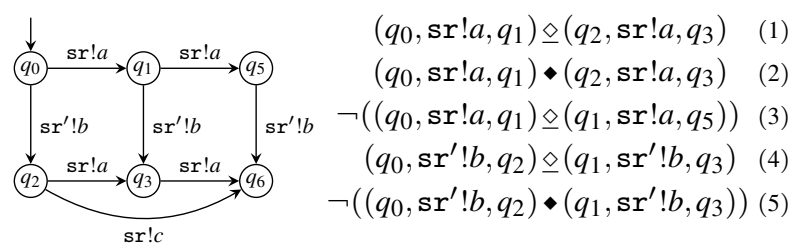

The relations in (1-2) hold since both transitions are interleaved with $\mathrm{sr}^{\prime} ! b$. The relation in (3) does not hold since the transition between the source of one $\left(q_{0}\right)$ and the source of the other $\left(q_{1}\right)$ passes through sr! $a$ itself. The two transitions in (3) are sequential rather than concurrent. The relation in (4) holds, but the relation in (5) does not because there is $\left(q_{5}, \mathrm{sr}^{\prime} ! b, q_{6}\right)$ in the $\diamond$-equivalence classes of $\left(q_{0}, \mathrm{sr}^{\prime} ! b, q_{2}\right)$ for which the condition does not hold (due to the transition with label $s r ! c)$.

In Figure 1. $\left(\mathrm{C}_{0}, \mathrm{AC}\right.$ ?cwin, $\left.\mathrm{C}_{1}\right) \bullet\left(\mathrm{C}_{2}, \mathrm{AC}\right.$ ?cwin, $\left.\mathrm{C}_{4}\right)$ since both transitions represent the same action interleaved with CD!busy. In each machine in Figure 1, a set of transitions $\left(q, \ell, q^{\prime}\right)$ with the same label $\ell$ forms a -equivalence class, e.g., in Alice, $\left\{\left(\mathrm{A}_{1}, \mathrm{CA}\right.\right.$ ?msg, $\left.\mathrm{A}_{3}\right),\left(\mathrm{A}_{2}, \mathrm{CA}\right.$ ?msg, $\left.\left.\mathrm{A}_{4}\right)\right\}$ is a -equivalence class labelled by CA?msg.

\section{CFSMs Characterisation of Global Graphs}

\subsection{Synchronous transition system}

Systems amenable to be transformed into global graphs are identified through their synchronous transition system (cf. Definition 3.2 where nodes consist of a vector of local states and transitions are labelled by elements in the set of events $\mathcal{E} \stackrel{\text { def }}{=} \bigcup_{\mathrm{s}, \mathrm{r} \in \mathcal{P}} Q_{\mathrm{s}} \times$ $\left.Q_{\mathrm{r}} \times\{(\mathbf{s}, \mathbf{r})\} \times \mathbb{A}\right\}$. Intuitively, an event $\left(q_{\mathrm{s}}, q_{\mathrm{r}}, \mathrm{s}, \mathrm{r}, a\right) \in \mathcal{E}$, written $\left(q_{\mathrm{s}}, q_{\mathrm{r}}, \mathrm{s} \rightarrow \mathrm{r}: a\right)$ for short, indicates that machines $\mathrm{s}$ and $\mathrm{r}$ can exchange message $a$ when they are respectively in state $q_{\mathrm{s}}$ and $q_{\mathrm{r}}$. Indexing events with the local states of the machines permits to distinguish two occurrences of the same communication at two different points in a global graph. To single out parallelism at the machine level, we introduce an equivalence relation over events that identifies events whose underlying local transitions are •-equivalent.

Definition 3.1 ( $\mathcal{E}$-equivalence). The event equivalence is the relation $\bowtie \stackrel{\text { def }}{=} \bowtie_{\mathrm{s}} \cap \bowtie_{\mathrm{r}} \subseteq \mathcal{E} \times \mathcal{E}$ where

$$
\begin{aligned}
& \left(q_{1}, q_{2}, \mathrm{~s} \rightarrow \mathrm{r}: a\right) \bowtie_{\mathrm{s}}\left(q_{1}^{\prime}, q_{2}^{\prime}, \mathrm{s} \rightarrow \mathrm{r}: a\right) \Longleftrightarrow \\
& \forall\left(q_{1}, \mathrm{sr} ! a, q_{3}\right),\left(q_{1}^{\prime}, \mathrm{sr} ! a, q_{3}^{\prime}\right) \in \delta_{\mathrm{s}}:\left(q_{1}, \mathrm{sr} ! a, q_{3}\right) \bullet\left(q_{1}^{\prime}, \mathrm{sr} ! a, q_{3}^{\prime}\right) \\
& \left(q_{1}, q_{2}, \mathrm{~s} \rightarrow \mathrm{r}: a\right) \bowtie_{\mathrm{r}}\left(q_{1}^{\prime}, q_{2}^{\prime}, \mathrm{s} \rightarrow \mathrm{r}: a\right) \Longleftrightarrow \\
& \forall\left(q_{2}, \mathrm{sr} ? a, q_{4}\right),\left(q_{2}^{\prime}, \mathrm{sr} ? a, q_{4}^{\prime}\right) \in \delta_{\mathrm{r}}:\left(q_{2}, \mathrm{sr} ? a, q_{4}\right) \bullet\left(q_{2}^{\prime}, \mathrm{sr} ? a, q_{4}^{\prime}\right)
\end{aligned}
$$

We let $[e]$ denote the $\bowtie$-equivalence class of event $e$. $\diamond$

Example 3.1. Consider the communicating system below:
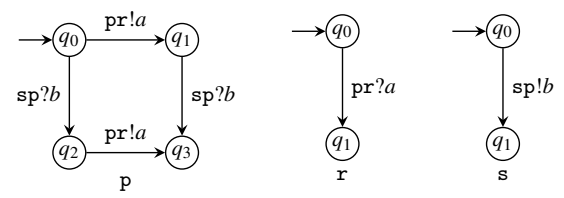


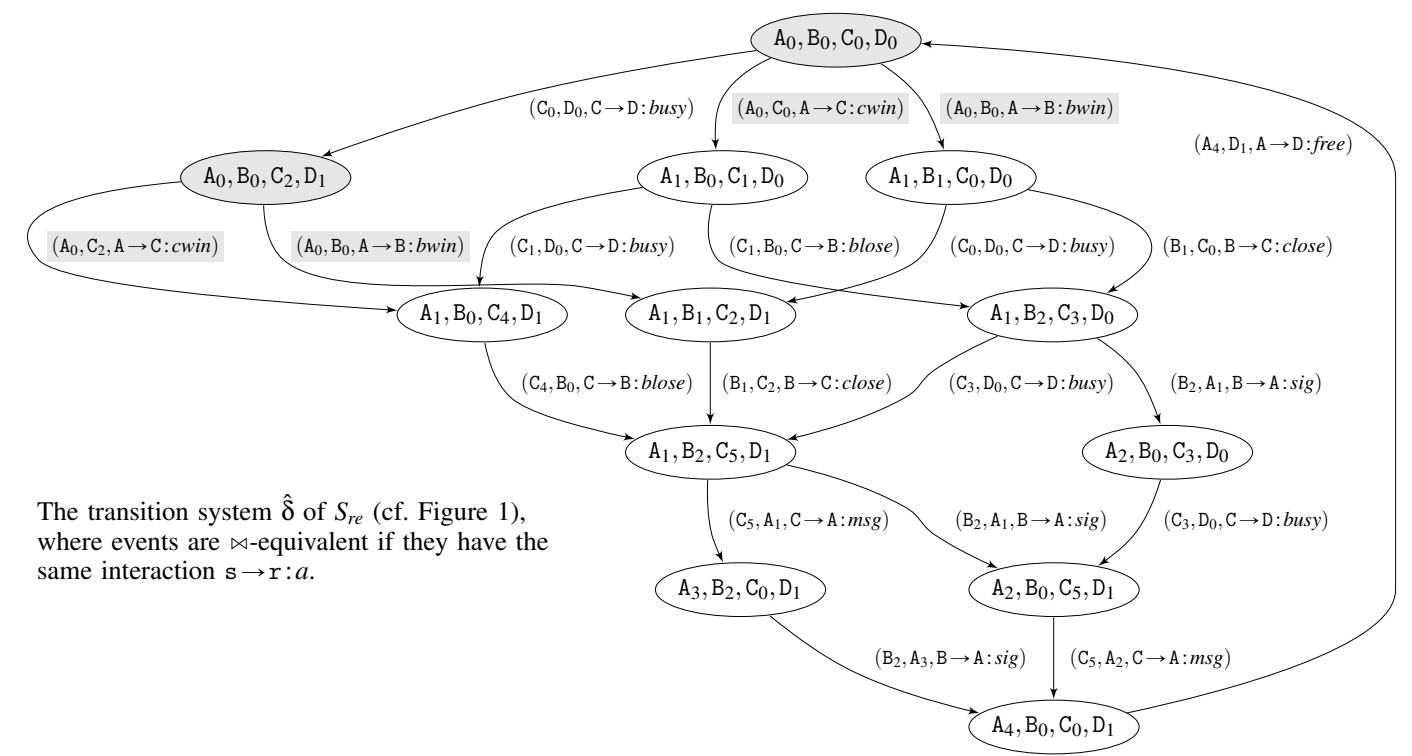

Figure 3. Transition graph of $\hat{\delta}$ and $T S\left(S_{r e}\right)$

Its synchronous transition system (cf. Definition 3.2 below) is the labelled transition system:

$$
\left(q_{0}, q_{0}, \mathrm{~s} \rightarrow \mathrm{p}: b\right) \underbrace{}_{\left(q_{2}, q_{0}, \mathrm{p} \rightarrow \mathrm{r}: a\right)} \longrightarrow 0
$$

We have $\left(q_{0}, q_{0}, \mathrm{p} \rightarrow \mathrm{r}: a\right) \bowtie\left(q_{2}, q_{0}, \mathrm{p} \rightarrow \mathrm{r}: a\right)$ and $\left(q_{0}, q_{0}, \mathrm{~s} \rightarrow \mathrm{p}\right.$ : b) $\bowtie\left(q_{0}, q_{3}, \mathrm{~s} \rightarrow \mathrm{p}: b\right)$. Considering equivalent the events on the "vertical" transitions and those on the "horizontal" ones equivalent allows us to identify a pair of concurrent interactions; while still differentiating them from other occurences of communications $\mathrm{p} \rightarrow$ $\mathrm{r}: a$ and $\mathrm{s} \rightarrow \mathrm{p}: b$.

In our running example (cf. Figure 1 , we have $\left(C_{5}, A_{2}, C \rightarrow\right.$ $\mathrm{A}: m s g) \bowtie\left(\mathrm{C}_{5}, \mathrm{~A}_{1}, \mathrm{C} \rightarrow \mathrm{A}: m s g\right)$ since the underlying transitions of $A$ are --equivalent, i.e., $\left(\mathrm{A}_{1}, \mathrm{CA}\right.$ ? $\left.m s g, \mathrm{~A}_{3}\right) \bullet\left(\mathrm{A}_{2}, \mathrm{CA}\right.$ ? $\left.m s g, \mathrm{~A}_{4}\right)$, and the underlying transition of $\mathrm{C}$ is the same for both events, i.e., $\left(\mathrm{C}_{5}, \mathrm{CA}\right.$ ! msg, $\left.\mathrm{C}_{0}\right)$.

Hereafter, we let $n, n^{\prime}, \ldots$ denote vectors of local states and $n[\mathrm{p}]$ denote the state of $\mathrm{p} \in \mathcal{P}$ in $n$.

Definition 3.2 (Synchronous transition system). Given a system $S=\left(M_{\mathrm{p}}\right)_{\mathrm{p} \in \mathcal{P}}$, let $N \stackrel{\text { def }}{=}\left\{\vec{q} \mid(\vec{q} ; \vec{\varepsilon}) \in R S_{1}(S)\right\}$,

$\hat{\delta} \stackrel{\text { def }}{=}\left\{\left(n, e, n^{\prime}\right) \mid(n ; \vec{\varepsilon}) \stackrel{\mathrm{sr} ! a}{\longrightarrow} \stackrel{\mathrm{sr} ? a}{\longrightarrow}\left(n^{\prime} ; \vec{\varepsilon}\right) \wedge e=(n[\mathrm{~s}], n[\mathrm{r}], \mathrm{s} \rightarrow \mathrm{r}: a)\right\}$ and $E \stackrel{\text { def }}{=}\left\{e \mid \exists n, n^{\prime} \in N:\left(n, e, n^{\prime}\right) \in \hat{\delta}\right\} \subseteq \mathcal{E}$. The synchronous transition system of $S$ is $T S(S)=\left(N, n_{0}, E / \bowtie, \Longrightarrow\right)$ where $n_{0}=\vec{q}_{0}$ is the initial state, and $n \stackrel{[e]}{\Longrightarrow} n^{\prime} \Longleftrightarrow\left(n, e, n^{\prime}\right) \in \hat{\delta}$. We fix a set $\hat{E}$ of representative elements of each $\bowtie$-equivalence class (i.e., $\hat{E} \subseteq E$ and $\left.\forall e \in E \exists ! e^{\prime} \in \hat{E}: e^{\prime} \in[e]\right)$ and write $n \stackrel{e^{\prime}}{\Longrightarrow} n^{\prime}$ for $n \stackrel{[e]}{\Rightarrow} n^{\prime}$ when $e^{\prime} \in[e] \cap \hat{E}$. Sequences of events are ranged over by $\pi$ and we extend the notation on $\rightarrow$ in Definition 2.3 to $\Longrightarrow$ (e.g., if $\pi=e_{1} \cdots e_{k}, n_{1} \stackrel{\pi}{\Rightarrow} n_{k+1}$ iff $\left.n_{1} \stackrel{e_{1}}{\Rightarrow} n_{2} \stackrel{e_{2}}{\Longrightarrow} \cdots \stackrel{e_{k}}{\Rightarrow} n_{k+1}\right)$.

$T S(S)$ represents all the possible synchronous executions of system $S$; and each transition is labelled by an event $e$, taken up-to equivalence so to distinguish different occurrences of a same com- munication, while preserving the parallelism of local machines. The synchronous transition system for our running example is given in Figure 3

Definition 3.3 (Projections). The projection of an event $e$ onto participant $\mathrm{p}$, denoted by $e l_{\mathrm{p}}$, is defined as follows:

$$
\left(q_{\mathrm{s}}, q_{\mathrm{r}}, \mathrm{s} \rightarrow \mathrm{r}: a\right) l_{\mathrm{p}} \stackrel{\text { def }}{=} \begin{cases}\mathrm{pr} ! a & \text { if } \mathrm{s}=\mathrm{p} \\ \mathrm{sp} ? a & \text { if } \mathrm{r}=\mathrm{p} \\ \varepsilon & \text { otherwise }\end{cases}
$$

Projection is defined on sequences of events in the obvious way. The projection of $T S(S)=\left(N, n_{0}, \hat{E}, \Longrightarrow\right)$ on participant $\mathrm{p}$, written $T S(S) \iota_{\mathrm{p}}$, is the automaton $\left(Q, q_{0}, \mathbb{A}, \delta\right)$ where $Q=N, q_{0}=n_{0}$, and $\delta \subseteq Q \times A c t \cup\{\varepsilon\} \times Q$ is s.t. $\left(n_{1},\left.e\right|_{\mathrm{p}}, n_{2}\right) \in \delta \Longleftrightarrow n_{1} \stackrel{e}{\Rightarrow} n_{2} . \diamond$

\subsection{Generalised multiparty compatibility}

We introduce generalised multiparty compatibility (GMC) as a sound and complete condition for constructing global graphs. Hereafter, we fix a system $S=\left(M_{\mathrm{p}}\right)_{\mathrm{p} \in \mathcal{P}}$ with $T S(S)=\left(N, n_{0}, \hat{E}, \Longrightarrow\right)$. Essentially, GMC relies on two conditions, (1) representability (cf. Definition 3.4): for each machine, each trace and each choice are represented in $T S(S)$; and (2) branching property (Definition 3.5): whenever there is a choice in $T S(S)$, a unique machine takes the decision and each of the other participants is either made aware of which branch was chosen or not involved in the choice. Representability guarantees that $T S(S)$ contains enough information to decide safety properties of any (asynchronous) execution of $S$; and the branching property ensures that, if a branching in $T S(S)$ represents a choice, then this choice is "well-formed".

For a language $\mathcal{L}, h d(\mathcal{L})$ returns the first actions of $\mathcal{L}$ (if any).

$$
h d(\mathcal{L}) \stackrel{\text { def }}{=}\left\{\ell \mid \exists \varphi \in A c t^{*}: \ell \cdot \varphi \in \mathcal{L}\right\} \quad h d(\{\varepsilon\}) \stackrel{\text { def }}{=}\{\varepsilon\}
$$

Given $n \in N$, let $T S(S)\langle n\rangle$ be the transition system $T S(S)$ where the initial state $n_{0}$ is replaced by $n$. We write $L T(S, n, \mathrm{p})$ for $\mathcal{L}\left(T S(S)\langle n\rangle \ell_{\mathrm{p}}\right)$; that is $L T(S, n, \mathrm{p})$ is the language obtained by setting the initial node of $T S(S)$ to $n$ and then projecting this new transition system onto $\mathrm{p}$.

Definition 3.4 (Representability). System $S$ is representable if 
1. $\mathcal{L}\left(M_{\mathrm{p}}\right)=L T\left(S, n_{0}, \mathrm{p}\right)$ and

2. $\forall q \in Q_{\mathrm{p}} \exists n \in N: n[\mathrm{p}]=q \wedge \bigcup_{\left(q, \ell, q^{\prime}\right) \in \delta_{\mathrm{p}}}\{\ell\} \subseteq h d(L T(S, n, \mathrm{p}))$. for all $\mathrm{p} \in \mathcal{P}$.

Condition (1) in Definition 3.4 is needed to ensure that each trace of each machine is represented in $T S(S)$; while condition (2) is necessary to ensure that every choice in each machine is represented in $T S(S)$.

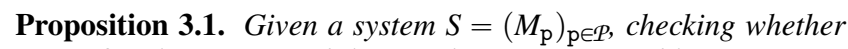
$S$ satisfies the representability condition is computable in

$$
O\left(\sum_{\mathrm{p} \in \mathcal{P}} 2^{|N|+\left|Q_{\mathrm{p}}\right|}\right) \text { time, with }|N|=\prod_{\mathrm{p} \in \mathcal{P}}\left|Q_{\mathrm{p}}\right|
$$

In the worst case, the time complexity of checking the representability of $S$ is exponential. This is solely due to the language equivalence check (condition (1) in Definition 3.4) between each machine and its projection from $T S(S)$. However, as observed in [12], in practice algorithms for language equivalence behave very efficiently. In addition, we can remove some states from the projection of $T S(S)$, e.g., those that are on chains of $\varepsilon$-transitions only, while preserving its language, thus reducing the exponent $|N|$.

We give a few auxiliary definitions before formalising the branching property. For $n \neq n^{\prime} \in N$, we define $n \prec n^{\prime}$ iff $n \Longrightarrow{ }^{*} n^{\prime}$ and for all paths $n_{0} \Longrightarrow n_{1} \Longrightarrow \ldots \Longrightarrow n_{k-1} \Longrightarrow n_{k}=n$ in $T S(S)$ such that $n_{0}, \ldots, n_{k}$ are pairwise distinct, $n^{\prime} \neq n_{h}$ for all $0 \leqslant h \leqslant k$. Intuitively, $n<n^{\prime}$ holds if $n^{\prime}$ is reachable from $n$ and no simple path from $n_{0}$ to $n$ goes through $n^{\prime}$; note that $\prec$ is not a preorder in general. The last nodes reachable from $n \in N$ with $e_{1} \neq e_{2} \in \hat{E}$ are

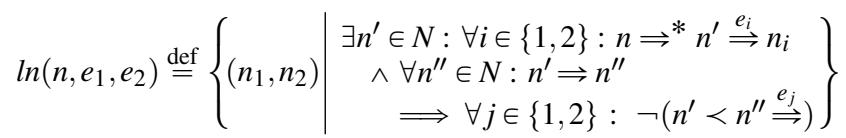

If $\left(n_{1}, n_{2}\right) \in \ln \left(n, e_{1}, e_{2}\right)$, then $n_{i}$ is a $\stackrel{e_{i}}{\Rightarrow}$-successor $(i=1,2)$ of a node $n^{\prime}$ on a path from $n$ whose successors are either not able to fire both $e_{1}$ and $e_{2}$ or not $\prec$-related to $n^{\prime}$.

Example 3.2. Consider the synchronous transition system below.

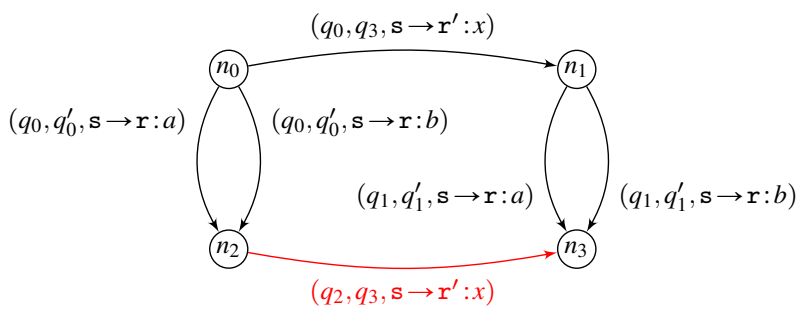

If $q_{0}=q_{1}$ and $q_{0}^{\prime}=q_{1}^{\prime}$, we have $\ln \left(n_{0},\left(q_{0}, q_{0}^{\prime}, \mathrm{s} \rightarrow \mathrm{r}: a\right),\left(q_{0}, q_{0}^{\prime}, \mathrm{s} \rightarrow\right.\right.$ $r: b))=\left\{\left(n_{3}, n_{3}\right)\right\}$. In this case, both branches on $a$ and $b$ from nodes $n_{0}$ and $n_{1}$ are considered equivalent (they are only interleaved with the exchange of message $x$ ). However, if the edge from $n_{2}$ to $n_{3}$ is removed and $q_{0} \neq q_{1}$ and $q_{0}^{\prime} \neq q_{1}^{\prime}$, then $\ln \left(n_{0},\left(q_{0}, q_{0}^{\prime}, \mathrm{s} \rightarrow\right.\right.$ $\left.\mathrm{r}: a),\left(q_{0}, q_{0}^{\prime}, \mathrm{s} \rightarrow \mathrm{r}: b\right)\right)=\left\{\left(n_{2}, n_{2}\right)\right\}$. In this case the two branches are not equivalent since one of them prevents $x$ to be ever exchanged.

In our running example (cf. Figure 3, we have:

$$
\begin{array}{r}
\ln \left(\left(\mathrm{A}_{0}, \mathrm{~B}_{0}, \mathrm{C}_{0}, \mathrm{D}_{0}\right),\left(\mathrm{A}_{0}, \mathrm{~B}_{0}, \mathrm{~A} \rightarrow \mathrm{B}: \text { bwin }\right),\left(\mathrm{A}_{0}, \mathrm{C}_{0}, \mathrm{~A} \rightarrow \mathrm{C}: c \text { win }\right)\right) \\
=\left\{\left(\left(\mathrm{A}_{1}, \mathrm{~B}_{1}, \mathrm{C}_{2}, \mathrm{D}_{1}\right),\left(\mathrm{A}_{1}, \mathrm{~B}_{0}, \mathrm{C}_{4}, \mathrm{D}_{1}\right)\right)\right\}
\end{array}
$$

Recall that $\left(\mathrm{A}_{0}, \mathrm{C}_{2}, \mathrm{~A} \rightarrow \mathrm{C}\right.$ : cwin $) \bowtie\left(\mathrm{A}_{0}, \mathrm{C}_{0}, \mathrm{~A} \rightarrow \mathrm{C}\right.$ : cwin $)$; i.e., the pair of events can be fired from both $\left(A_{0}, B_{0}, C_{0}, D_{0}\right)$ and $\left(A_{0}, B_{0}, C_{2}, D_{1}\right)$.
For an event $e=\left(q_{\mathrm{s}}, q_{\mathrm{r}}, \mathrm{s} \rightarrow \mathrm{r}: a\right) \in \mathcal{E}$, let $\mathrm{l}(e)=\mathrm{s} \rightarrow \mathrm{r}: a$ and define a dependency relation $\triangleleft \subseteq \mathcal{E} \times \mathcal{E}$ on events:

$e \triangleleft e^{\prime} \Longleftrightarrow \mathrm{l}(e)=\mathrm{s} \rightarrow \mathrm{r}: a \wedge\left(\mathrm{l}\left(e^{\prime}\right)=\mathrm{s} \rightarrow \mathrm{r}: a^{\prime} \vee \mathrm{l}\left(e^{\prime}\right)=\mathrm{r} \rightarrow \mathrm{r}^{\prime}: a^{\prime}\right)$ Intuitively, $e$ and $e^{\prime}$ are $\triangleleft$-related if there exists a dependency relation between the two interactions, from the point of view of the receiver. We define a relation $e \triangleleft e^{\prime}$ in $\pi$ if there is a $\triangleleft$-relation between $e$ and $e^{\prime}$ in $\pi$, i.e.,

$e \triangleleft e^{\prime}$ in $\pi \Longleftrightarrow \begin{cases}\left(e \triangleleft e^{\prime \prime} \wedge e^{\prime \prime} \triangleleft e^{\prime} \text { in } \pi^{\prime}\right) \vee e \triangleleft e^{\prime} \text { in } \pi^{\prime} & \text { if } \pi=e^{\prime \prime} \cdot \pi^{\prime} \\ e \triangleleft e^{\prime} & \text { otherwise }\end{cases}$ also, $\operatorname{dep}\left(\mathrm{l}(e), \pi, \mathrm{l}\left(e^{\prime}\right)\right)$ iff

$$
\begin{aligned}
\left(\pi=\pi_{1} \cdot e \cdot \pi_{2} \cdot e^{\prime} \cdot \pi^{\prime}\right. & \left.\wedge\left({ }_{-},, \mathrm{l}(e)\right) \notin \pi_{1} \wedge\left({ }_{-},{ }_{-}, \mathrm{l}\left(e^{\prime}\right)\right) \notin \pi_{2}\right) \\
& \Longrightarrow e \triangleleft e^{\prime} \text { in } \pi_{2}
\end{aligned}
$$

which checks whether there is a dependency between two interactions on a path $\pi$ (if these interactions do appear in $\pi$ ). Below we give the second condition for GMC, which ensures that each "global choice" is made by exactly one participant and that all the other participants are either made aware of the choice or not involved in/affected by the choice.

Definition 3.5 (Branching property). System $S$ has the branching property if for all $n \in N$ and for all $e_{1} \neq e_{2} \in \hat{E}$ such that $n \stackrel{e_{1}}{\Rightarrow} n_{1}$ and $n \stackrel{e_{2}}{\Rightarrow} n_{2}$, then we have that

1. either there is $n^{\prime} \in N$ such that $n_{1} \stackrel{e_{2}}{\Longrightarrow} n^{\prime}$ and $n_{2} \stackrel{e_{1}}{\Longrightarrow} n^{\prime}$, or

2. for each $\left(n_{1}^{\prime}, n_{2}^{\prime}\right) \in \ln \left(n, e_{1}, e_{2}\right)$, letting

$$
L_{\mathrm{p}}^{i} \stackrel{\text { def }}{=} h d\left(\left\{e_{i} l_{\mathrm{p}} \cdot \varphi \mid \varphi \in L T\left(S, n_{i}^{\prime}, \mathrm{p}\right)\right\}\right) \text { with } i \in\{1,2\} \text { and } \mathrm{p} \in \mathcal{P},
$$

conditions 2a, 2b, and (2c) below hold.

(a) choice-awareness: $\forall \mathrm{p} \in \mathcal{P}$ : either

$$
\begin{aligned}
& \text { i. } L_{\mathrm{p}}^{1} \cap L_{\mathrm{p}}^{2} \subseteq\{\varepsilon\} \text { and } \varepsilon \in L_{\mathrm{p}}^{1} \Longleftrightarrow \varepsilon \in L_{\mathrm{p}}^{2} \text {, or } \\
& \text { ii. } \exists n^{\prime} \in N, \pi_{1}, \pi_{2}: \\
& \quad n_{1}^{\prime} \stackrel{\pi_{1}}{\Rightarrow} n^{\prime} \wedge n_{2}^{\prime} \stackrel{\pi_{2}}{\Rightarrow} n^{\prime} \wedge\left(e_{1} \cdot \pi_{1}\right) l_{\mathrm{p}}=\left(e_{2} \cdot \pi_{2}\right) l_{\mathrm{p}}=\varepsilon
\end{aligned}
$$

(b) unique selector: $\exists$ ! $\mathrm{s} \in \mathcal{P}: L_{\mathrm{s}}^{1} \cap L_{\mathrm{s}}^{2}=\varnothing \wedge \exists \mathrm{sr} ! a \in L_{\mathrm{s}}^{1} \cup L_{\mathrm{s}}^{2}$

(c) no race: $\forall \mathrm{r} \in \mathcal{P}: L_{\mathrm{r}}^{1} \cap L_{\mathrm{r}}^{2}=\varnothing$

$$
\begin{aligned}
& \Longrightarrow \forall \mathrm{s}_{1} \mathrm{r} ? a_{1} \in L_{\mathrm{r}}^{1}, \forall \mathrm{s}_{2} \mathrm{r} ? a_{2} \in L_{\mathrm{r}}^{2}: \forall i \neq j \in\{1,2\}: n_{i}^{\prime} \stackrel{\pi_{i}}{\Rightarrow} \\
& \Longrightarrow \operatorname{dep}\left(\mathrm{s}_{i} \rightarrow \mathrm{r}: a_{i}, e_{i} \cdot \pi_{i}, \mathrm{~s}_{j} \rightarrow \mathrm{r}: a_{j}\right)
\end{aligned}
$$

Definition 3.5 ensures that every branching either is (1) the concurrent execution of two events; or, for each participant $p$, (2(a)i) if $\mathrm{p}$ does not terminates before $n$, then the first actions of $p$ in two different branches are disjoint; or 2(a)ii $p$ is not involved in the choice, i.e., the branches merge before $\mathrm{p}$ does any action; (2b) there is a unique participant s making the decision; and 2c for each participant $r$ involved in the choice, there cannot be a race condition between the messages that $r$ can receive. The no race condition notably ensures that in any (asynchronous) execution of $S$, if a machine has more than one non-empty buffers, then it can read from them in any order (interleaving is possible). Note that if a machine $r$ receives all its messages from a same sender, then there is a $\triangleleft$-relation between all its actions.

In system $S_{r e}$, case (1) of Definition 3.5 applies to all branching nodes except $n_{0}=\left(\mathrm{A}_{0}, \mathrm{~B}_{0}, \mathrm{C}_{0}, \mathrm{D}_{0}\right)$ and $n=\left(\mathrm{A}_{0}, \mathrm{~B}_{0}, \mathrm{C}_{2}, \mathrm{D}_{1}\right)$, highlighted in Figure 3 for which case (2) applies. For $e_{1}=\left(\mathrm{A}_{0}, \mathrm{~B}_{0}, \mathrm{~A} \rightarrow\right.$ $\mathrm{B}:$ bwin $)$ and $e_{2}=\left(\mathrm{A}_{0}, \mathrm{C}_{0}, \mathrm{~A} \rightarrow \mathrm{C}:\right.$ cwin $)$, we have $\ln \left(n_{0}, e_{1}, e_{2}\right)=$ $\ln \left(n, e_{1}, e_{2}\right)=\left\{\left(\left(\mathrm{A}_{1}, \mathrm{~B}_{1}, \mathrm{C}_{2}, \mathrm{D}_{1}\right),\left(\mathrm{A}_{1}, \mathrm{~B}_{0}, \mathrm{C}_{4}, \mathrm{D}_{1}\right)\right)\right\}$. Hence, case 2a holds for $n_{0}$ iff it holds for $n$. Following (2a), we check that every participant satisfies either 2(a)i] or 2(a)ii):

- A executes different (sending) actions in both branches (AB!bwin and AC! ( win),

- B executes different (receiving) actions (AB?bwin and $\mathrm{CB}$ ?blose), 
- $\mathrm{C}$ executes different (receiving) actions (AC?cwin and $\mathrm{BC}$ ?close),

hence case 2(a)i applies to A, B, and C. While case 2(a)ii) applies to $\mathrm{D}$ since there is a node $n^{\prime}=\left(\mathrm{A}_{1}, \mathrm{~B}_{2}, \mathrm{C}_{5}, \mathrm{D}_{1}\right)$ such that $\mathrm{D}$ does not execute any action on either path from $n$ to $n^{\prime}$ (through nodes $\left(A_{1}, B_{1}, C_{2}, D_{1}\right)$ and $\left(A_{1}, B_{0}, C_{4}, D_{1}\right)$, respectively). Also, condition (2b) is satisfied since $A$ is the unique sender that executes different actions in both branches $e_{1}$ and $e_{2}$.

Condition $\sqrt{2 \mathrm{C}}$ is satisfied for $\mathrm{B}$ and $\mathrm{C}$ due to the existence of dependency chains from $A B$ ? bwin to $C B$ ?blose (and vice versa) and from $\mathrm{AC}$ ?cwin to $\mathrm{BC}$ ?close (and vice versa). For instance, the dependency chain $\mathrm{B} \rightarrow \mathrm{C}$ : close $\triangleleft \mathrm{C} \rightarrow \mathrm{A}:$ ms $\Rightarrow \triangleleft \mathrm{A} \rightarrow \mathrm{C}$ : cwin prevents $\mathrm{C}$ to delay the reception of close (sent by $\mathrm{B}$ ) until she can receive message $c$ win (sent by A); $\mathrm{C}$ must send a message $m s g$ (to A) before she can receive the outcome of a new round of the game.

Finally, note that $\ln \left(n_{0}, e_{1}, e_{2}\right)$ ensures that checking the branching between $e_{1}$ and $e_{2}$ at node $n_{0}$ is delayed until the interaction $\mathrm{C} \rightarrow \mathrm{D}:$ busy does not interfere with the choice. Hence, the behaviours of $\mathrm{C}$ and $\mathrm{D}$ are checked only once they have exchanged the busy message.

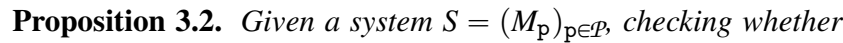
$S$ satisfies the branching property is computable in

$$
O\left(|\Longrightarrow|^{2} \times|\Longrightarrow| ! \times \sum_{r \in \mathcal{P}}\left(\left|\delta_{r}\right|^{2}\right)\right) \text { time. }
$$

Checking the branching property is factorial in the size of $T S(S)$ because it requires the enumeration of paths of $T S(S)$ (cf. 2c) of Definition 3.5. We remark that the above is a rather coarse approximation obtained under worst case assumptions oblivious of the typical structure of $T S(S)$; our experiments show good performances (cf. §5. Finally, we observe that $T S(S)$ is generally much smaller than, e.g., the one-bounded transition system of $S$ (where each queue may contain at most one message).

Definition 3.6 (Generalised multiparty compatibility). A system $S$ is generalised multiparty compatible $(G M C)$ if it is representable and has the branching property.

Example 3.3. We show the interplay between the representability and branching conditions by exhibiting unsafe systems satisfying only one of the properties. Consider the following machines:

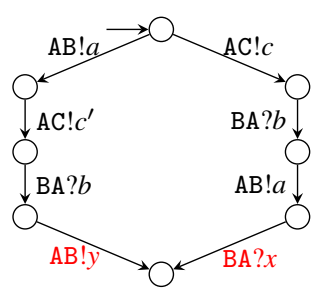

A

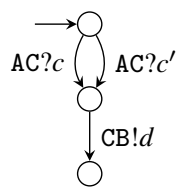

$\mathrm{C}_{1}$
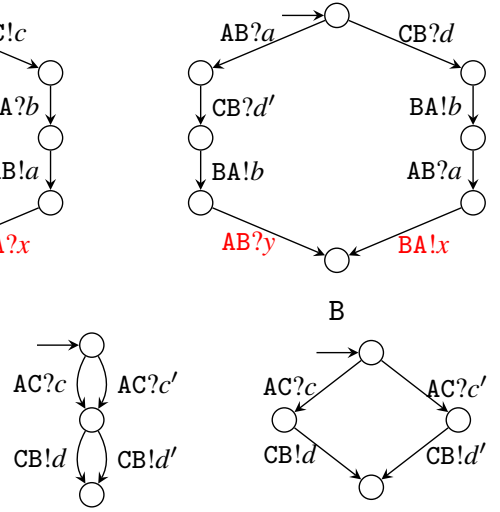

$\mathrm{C}_{2}$

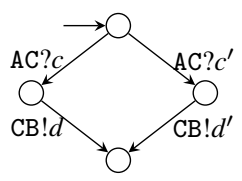

$\mathrm{C}_{3}$
(1) System $S_{1}=\left(\mathrm{A}, \mathrm{B}, \mathrm{C}_{1}\right)$ with $d=d^{\prime}$ is not safe: whenever the left-hand side branch of $A$ and the right-hand side branch of $B$ are taken in a same execution, $S_{1}$ will reach an orphan message configuration where messages $x$ and $y$ are never consumed. In fact, $S_{1}$ is not GMC because there is a branching node from which $B$ can execute, as first actions, either $\mathrm{AB} ? a$ or $\mathrm{CB} ? d$, and there is no dependency between the reception of $a$ and that of $d^{\prime}$ (with $d=d^{\prime}$ ) in the left-hand side branch, i.e., $\neg\left(\mathrm{A} \rightarrow \mathrm{B}: a \triangleleft \mathrm{A} \rightarrow \mathrm{C}: c^{\prime} \triangleleft \mathrm{C} \rightarrow \mathrm{B}: d^{\prime}\right)$. Thus the branching property does not hold.

(2) System $S_{2}=\left(\mathrm{A}, \mathrm{B}, \mathrm{C}_{2}\right)$ with $d \neq d^{\prime}$ is not safe: as before, whenever the left-hand side branch of $A$ and the right-hand side branch of $B$ are taken in a same execution this system reaches an orphan message configuration. These two branches are not mutually exclusive since $\mathrm{C}_{2}$ can receive $c^{\prime}$ then send $d$. This system is not GMC since there is no node in $T S\left(S_{2}\right)$ such that actions CB! $d$ and CB! $d^{\prime}$ are the first actions executed by C. Hence the representability condition does not hold.

(3) System $S_{3}=\left(\mathrm{A}, \mathrm{B}, \mathrm{C}_{3}\right)$ with $d \neq d^{\prime}$ is safe and is GMC. In $S_{3}$, the left-hand side branch of $A$ and the right-hand side branch of $B$ are always mutually exclusive, while in $S_{1}$ and $S_{2}$ they are only mutually exclusive in synchronous executions.

We remark that systems $S_{1}$ and $S_{2}$ may be easily changed so that they are "safe" in any $k$-bounded execution but not safe in a $k+1$ bounded execution. This may be done by making A and B exchange $k+1$ messages consecutively, e.g., by replacing every $\mathrm{AB} ! a$ (resp. $\mathrm{AB} ? a$ ) transitions in $\mathrm{A}$ (resp. B) by a sequence of $k+1$ transitions $\mathrm{AB} ! a_{i}\left(\right.$ resp. $\left.\mathrm{AB} ? a_{i}\right)$, for $1 \leqslant i \leqslant k+1$.

Theorem 3.1 (Soundness). If $S$ is GMC, then it is safe (no orphan message, deadlock, and unspecified reception configurations).

Theorem 3.1 says that no (asynchronous) execution of $S$ will result in an orphan message, deadlock or unspecified reception configurations. Relying on representability (every transition and branching in each machine is represented in $T S(S)$ ), the proof shows that, for each branching node $n$, the function $\ln \left(n, e_{1}, e_{2}\right)$ allows enough branches to be verified against the branching property. Then, it shows that any sent message is eventually received and that a machine in a receiving state eventually receives a message it expected, by Definition 3.5

Example 3.4. The unsafe system below has the branching property and validates condition (1) of Definition 3.4 but not condition (2). This system can reach an orphan message configuration, where messages $x$ and $y$ are never received.

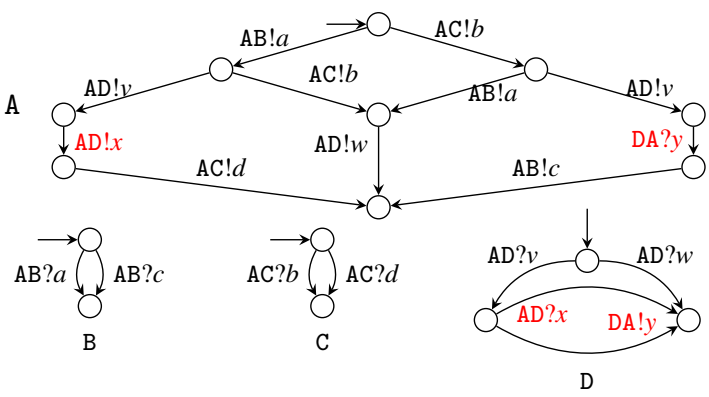

This example illustrates the importance of condition (2) of Definition 3.4 to ensure safety. In the $T S$ of this system (isomorphic to machine A), the branches corresponding to DA! $y$ and $\mathrm{AD} ? x$ of machine $\mathrm{D}$ are not checked against each other for the branching property.

\subsection{Amending communicating systems}

When a system is not GMC, our algorithm can be used to suggest different ways of transforming it, so to validate the condition. By Definition 3.6 we first note:

Proposition 3.3. If $S$ satisfies all but (1) in Definition 3.4 then the system consisting of the (minimised) projections of TS(S) is GMC.

This means that, in such a case, a new safe system may be automatically obtained from the projections of TS. For instance, system $S_{2}$ in Example 3.3 is not GMC because (1) in Definition 3.4 does 


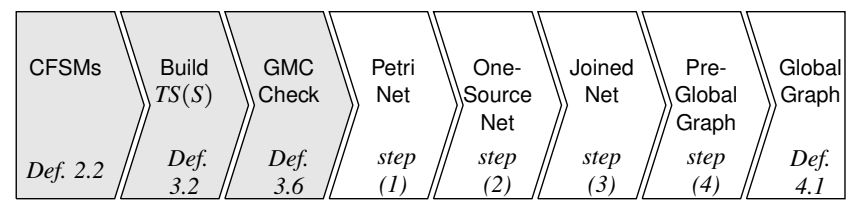

Figure 4. Work-flow of the construction

not hold. However, the system corresponding to the projections of $T S\left(S_{2}\right)$ is exactly system $S_{3}$, which is GMC.

In case the projections of $T S(S)$ do not provide a viable alternative, then the language equivalence check allows to highlight which transitions (or paths) of each machine are not represented in $T S(S)$. Similarly, local states and transitions violating it can be singled out, according to condition (2) in Definition 3.4 For instance, in Example 3.4 we can highlight all transitions over $x$ and $y$, as well as the states where they are enabled.

When the branching property (Definition 3.5 is violated, then our analysis permits to give precise information on where the problem occurs. First, we can give the vector of local states and the two branching events for which the problem occurs as well as a witnessing execution that leads to the offending configuration.

- If the choice-awareness condition $2 \mathrm{a}$ is violated, then we can list the machines for which the condition is not satisfied. If a machine has a first same receiving action in both branches, then it may be corrected by simply renaming some messages. These renamings can be automatically suggested while checking for the branching property. If the condition fails because a machine terminates in one branch but not in the other, then we can suggest to add a new label and a transition to the final state in the terminated branch; as well as a dual transition in a sending machine.

- If condition $2 \mathrm{2b}$ is violated, we can highlight the set of machines sending messages at this branching node. A solution may be found by identifying the genuine selecting machine and add communications from this machine to the others.

- If condition (2c) is violated, then we can highlight, for each machine violating the condition, on which messages a race condition may occur; and suggest to add an acknowledgement message between the two corresponding actions.

Note that since CFSMs are specification or abstraction of programs, it is generally not desirable to automatically repair non-GMC systems. Indeed, some corrections may not be reflected easily in the program or might have side effects in the corresponding implementation, analogously to concurrent programming where automatic corrections are not generally appealing, even if some deadlocks may be detected automatically (at run or compile time).

\section{Building Global Graphs}

In $\S 3$, we construct the synchronous transition system $T S(S)$ of a communicating system $S$, and check whether it is GMC. We now describe the construction algorithm and its properties; Figure 4 summarises the work-flow of the transformations.

The algorithm to construct a global graph $G$ from a synchronous transition system $T S(S)$ consists of the following steps:

(1) we apply the algorithm of Cortadella et al. [18] to derive a Petri net $\mathbb{N}$ from $T S(S)$;

(2) we transform $\mathbb{N}$ so that its initial marking consists of exactly one place;
(3) we join transitions whenever possible, so to make explicit join and fork points of the work-flow;

(4) we transform the net of (3) into a pre-global graph; finally, we "clean-up" the pre-global graph of unnecessary vertexes so to obtain a global graph.

For the sake of the presentation and because the transformations are rather mechanical, we explain them through our running example. The formal definitions of the transformations and additional results are given in Appendix B

For (1), it is enough for the reader to know that the algorithm in Cortadella et al. [18] is based on the theory of regions [7] and transforms a transition system into a safe and extended freechoice labelled Petri net, whose reachability graph is bisimilar to the original transition system. Basically, this algorithm transforms events of $T S(S)$ into transitions of $\mathbb{N}$ while the places are built out of regions, i.e., sets of states having a uniform behaviour wrt events. We assume in this section that each $T S(S)$ is self-loop fre ${ }^{2}$ i.e., $\forall n, n^{\prime} \in N: n \Longrightarrow n^{\prime} \Longrightarrow n \neq n^{\prime}$. The algorithm of [18] is applicable on a self-loop free $T S(S)$, since every event $e \in \hat{E}$ has an occurrence in $T S(S)$ by construction and every state $n$ is reachable from $n_{0}$, as stated in Lemma 4.1 below. The Petri net obtained from $T S\left(S_{r e}\right)$ in Figure 3 is given in Figure 5 (left).

Lemma 4.1. If $S$ is $G M C$ and $T S(S)=\left(N, n_{0}, \hat{E}, \Longrightarrow\right)$, then $\forall n \in$ $N: n_{0}{ }^{*} n$.

In step (2), we transform a Petri net obtained from Cortadella's algorithm into a Petri net whose initial marking consists of exactly one place. This allows us to construct a global graph that has a unique starting point. In our running example, the Petri net on the left of Figure 5 is transformed by adding a fresh place $\left(p_{0}\right)$, initially marked, and a fresh (silent) transition $\left(t_{0}\right)$ connected to places $p_{1}$ and $p_{2}$ (this simple transformation is not illustrated in Figure 5).

In step (3), a transformation ensures that parallel gates are used "as much as possible" in the graph (instead of mixing choice and parallel gates). In fact, the transformation joins sets of places that have the same preset or postset to minimise the number of choice gates. The Petri net in the middle of Figure 5 is the net obtained from the left-hand side net after applying step (2) and (3). In the second transformation, we add (i) $t_{1}$ and $p_{11}$ so to join $p_{1}$ and $p_{2}$ which have the same preset, i.e., $t_{0}$ and the transition with label $\left(\mathrm{A}_{4}, \mathrm{D}_{1}, \mathrm{~A} \rightarrow \mathrm{D}:\right.$ free $)$; and (ii) we add $t_{2}$ and $p_{10}$ so to join $p_{5}$ and $p_{6}$ which have the same preset, i.e., the transitions with labels $\left(\mathrm{C}_{1}, \mathrm{~B}_{0}, \mathrm{C} \rightarrow \mathrm{B}:\right.$ blose $)$ and $\left(\mathrm{B}_{1}, \mathrm{C}_{0}, \mathrm{~B} \rightarrow \mathrm{C}:\right.$ close $)$. Both $t_{1}$ and $t_{2}$ are silent transitions.

Let $\approx$ be the weak bisimilarity relation on reachability graphs (i.e., $\approx$ is the bisimilarity up-to silent transitions, cf. Appendix A.

Lemma 4.2. Let $\mathbb{N}_{1}$ be the Petri net obtained after step (1), let $\mathbb{N}_{2}$ (resp. $\mathbb{N}_{3}$ ) be obtained by applying step (2) (resp. (3)) to $\mathbb{N}_{1}$ (resp. $\left.\mathbb{N}_{2}\right)$. If $T_{i}$ is the reachability graph of $\mathbb{N}_{i}($ for $i=1,2,3)$ then $T_{1} \approx T_{2} \approx T_{3}$.

We now define global graphs (a superclass of the generalised global types of [19] that allows each gate to be connected to more than two predecessors or successors).

Definition 4.1 (Global graph). A global graph (over $\mathcal{P}$ and $\mathbb{A}$ ) is a labelled graph $\langle V, A, \Lambda\rangle$ with set of vertexes $V$, set of edges $A \subseteq V \times V$, and labelling function $\Lambda$ from $V$ to $\{0, \odot, \uparrow, \square\} \cup\{\mathrm{s} \rightarrow$ $\mathrm{r}: a \mid \mathrm{s}, \mathrm{r} \in \mathcal{P} \wedge a \in \mathbb{A}\}$ such that, $\Lambda^{-1}(\mathrm{O})$ is a singleton, and for each $v \in V$, if $\Lambda(v)$ is of the form $\mathrm{s} \rightarrow \mathrm{r}: a$ then $v$ has unique incoming

\footnotetext{
${ }^{2} \operatorname{In} T S(S)$, if an event $e$ self-loops, then any transition labelled by $e$ is a self-loop. Hence, we can easily lift the self-loop free assumption by decomposing each self-loop into two (pointed) transitions in $T S(S)$ and recompose them once the global graph is constructed.
} 

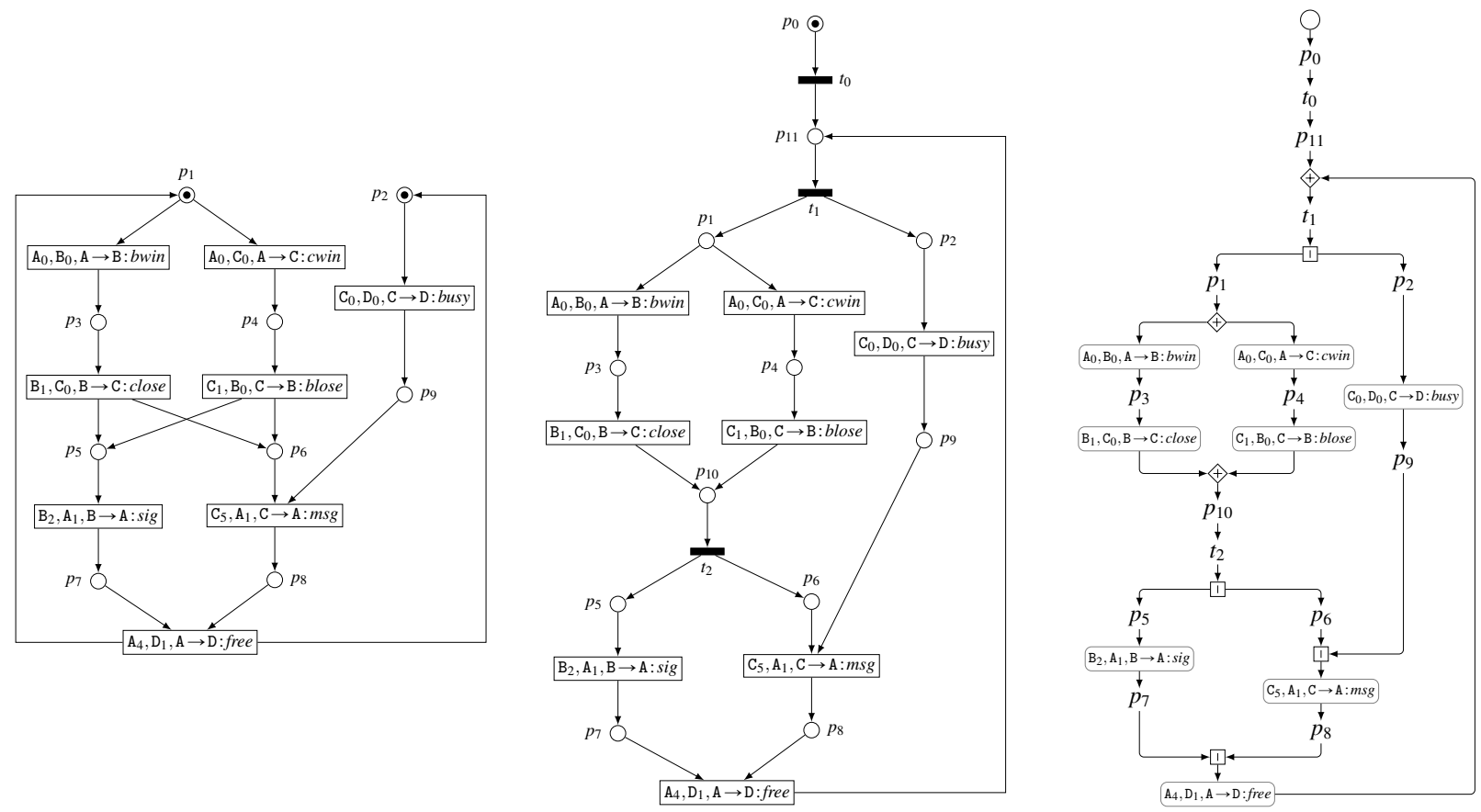

Figure 5. Derived net (left), net after transformations (middle), and pre-global graph (right)

and unique outgoing edges, and if $\Lambda(v) \in\{\leftrightarrow, \square\}, v$ has at least one incoming and one outgoing edge while $v$ has no outgoing edges if $\Lambda(v)=@$.

Label $\mathrm{s} \rightarrow \mathrm{r}: a$ represents an interaction where $\mathrm{s}$ sends a message $a$ to $r$. A vertex with label O represents the source of the global graph, @ represents the termination of a branch or of a thread, $\square$ indicates forking or joining threads, and $\uparrow$ marks vertexes corresponding to branch or merge points, or to entry points of loops.

In step (4), a pre-global graph is obtained from the Petri net obtained after step (3) via a transformation which consists in, firstly, creating a vertex in the global graph for each place, transition, and element of the flow relation. Then these vertexes are connected via gates: a source vertex is connected to a vertex without predecessor, a sink vertex is connected to any vertex without successors, while transitions (resp. places) are connected to a $\square$-gate (resp. $\uparrow$-gate) if they have more than one predecessors or successors. Finally, each component of the graph is connected by merging "ports" corresponding to elements of the flow relation. The pre-global graph for $S_{r e}$ (Figure 1) is given in Figure 5 (right).

A global graph is obtained from a pre-global graph by removing all unnecessary nodes (i.e., former places and transitions such as $p_{0}$ and $t_{0}$ in Figure 5) and relabelling events into interactions ( $e$ is replaced by $\mathrm{l}(e)$ ); e.g., the pre-global graph in Figure 5 becomes the global graph in Figure 2 .

Proposition 4.1. Steps (2) to (4) are computable in polynomial time in the size of $\mathbb{N}$.

We give the main result regarding the construction of a global graph from CFSMs. In Theorem 4.1 below, we formalise the relationship between the machines from which a global graph is constructed and its projections. Projecting a global graph $G$ can be done in two ways: (i) $G$ can be transformed into a Petri net whose reachability graph may be projected, similarly to the projection of $T S(S)$ (cf. Definition 3.3); or (ii) $G$ can be transformed into an au-

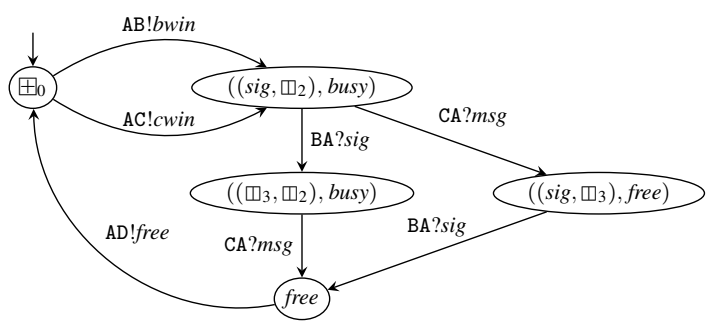

Figure 6. Projection of $G_{r e}$ onto A

tomaton whose states are the nodes of $G$ and each transition is labelled by $(\mathrm{s} \rightarrow \mathrm{r}: a) l_{\mathrm{p}}$ if the source state corresponds to a vertex with label $\mathrm{s} \rightarrow \mathrm{r}: a$, and by $\varepsilon$ otherwise. In order to recover local concurrency, we take the parallel composition of the automata resulting of the projection of each successor of a $\square$-gate. Finally, the resulting automaton is minimised wrt. language equivalence. We write $G \iota_{p}$ for the projection of $G$ onto p, and give the formal definition in [?]. As an example, Figure 6 shows the minimised projection of $G_{r e}$ (cf. Figure 2 ) onto A.

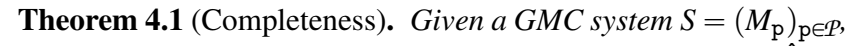
let $G$ be the global graph built from $S$ and let $T S(S)=\left(N, n_{0}, \hat{E}, \Longrightarrow\right.$ ). If $T S(S)$ is self-loop free (i.e. $\left.\forall n, n^{\prime} \in N: n \Longrightarrow n^{\prime} \Longrightarrow n \neq n^{\prime}\right)$, then $S$ is isomorphic to $\left(G \downarrow_{\mathrm{p}}\right)_{\mathrm{p} \in \mathcal{P}}$, the system made of the projection of $G$.

The proof of Theorem 4.1 (cf. [? ]) relies on the fact that each machine is preserved during the construction, i.e., (1) the projection of $T S(S)$ onto each $\mathrm{p}$ is language equivalent with $M_{\mathrm{p}}$, (2) the net obtained from $T S(S)$ via the algorithm in [18] is bisimilar to $T S(S)$, (3) each transformation preserves (weak) bisimilarity with 


\begin{tabular}{l|cccccc}
\multicolumn{1}{c}{$S$} & $|\mathcal{P}|$ & $|N|$ & $|\Rightarrow|$ & GMC & $|G|$ & Time (s) \\
\hline Running Example & 4 & 12 & 19 & $\checkmark$ & 16 & 0.184 \\
Running Example $\times 2$ & 8 & 144 & 456 & $\checkmark$ & 32 & 22.307 \\
Bargain & 3 & 4 & 4 & $\checkmark$ & 8 & 0.103 \\
Bargain $\times 2$ & 6 & 16 & 32 & $\checkmark$ & 16 & 0.161 \\
Alternating 2-bit [19] & 2 & 8 & 12 & $\checkmark$ & 13 & 0.161 \\
Alternating 2-bit $\times 2$ & 4 & 64 & 192 & $\checkmark$ & 24 & 0.355 \\
Alternating 3-bit [19] & 2 & 24 & 48 & $\checkmark$ & 18 & 3.164 \\
Alternating 3-bit $\times 2$ & 4 & 576 & 2304 & $\checkmark$ & 34 & 12.069 \\
TPMContract v2 [22] & 2 & 5 & 8 & $\checkmark$ & 15 & 0.142 \\
TPMContract v2 $\times 2$ & 4 & 25 & 80 & $\checkmark$ & 30 & 0.362 \\
Sanitary Agency [30] & 4 & 17 & 21 & $\checkmark$ & 22 & 0.241 \\
Sanitary Agency $\times 2$ & 8 & 196 & 476 & $\checkmark$ & 44 & 3.165 \\
Health System [14] & 6 & 10 & 11 & $\checkmark$ & 14 & 0.17 \\
Health System $\times 2$ & 12 & 100 & 220 & $\checkmark$ & 28 & 1.702 \\
Filter Collaboration [32] & 2 & 3 & 5 & $\checkmark$ & 10 & 0.118 \\
Filter Collaboration $\times 2$ & 4 & 9 & 30 & $\checkmark$ & 20 & 0.178 \\
Logistic [1] & 4 & 13 & 17 & $\checkmark$ & 27 & 0.276 \\
Logistic $\times 2$ & 8 & 169 & 442 & $\checkmark$ & 54 & 2.155 \\
Cloud System v4 [21] & 4 & 7 & 8 & $\checkmark$ & 12 & 0.14 \\
Cloud System v4 $\times 2$ & 8 & 49 & 112 & $\checkmark$ & 24 & 0.432
\end{tabular}

Table 1. Experiment results; $|\mathcal{P}|$ is the number of machines, $|N|$ (resp. $|\Longrightarrow|$ ) is the number of nodes (resp. transitions) in $T S(S)$, and $|G|$ is the number of vertices in $G$.

the derived net, cf. Lemma 4.2 and (4) the transformation to a global graph is sound since the net is extended free choice.

\section{Implementation and Experimental Evaluation}

In order to assess the applicability of our work and to estimate the effectiveness of checking for the GMC condition as well as constructing a global graph, we have developed a prototype tool supporting our theory [2]. The tool (implemented in Haskell) takes as input a textual representation of a communicating system $S$, then builds $T S(S)$ on which the representability condition and branching property are concurrently checked for (using HKC [12] to check for language equivalence). Then the tool constructs a global graph from $T S(S)$ relying on Petrify [3] (to derive a Petri net from $T S(S)$ ), and Graphviz (to render global graphs).

Table 1 summarises the results of experiments conducted on a few real-world protocols mainly taken from the literature. For each protocol, the table reports the number of machines, the number of nodes and transitions in $T S(S)$, whether it validates the GMC condition, the size of the constructed global graph, and the time it takes to check the condition and render its global graph (executions were on a $3.40 \mathrm{GHz}$ Intel i7 CPU with $16 \mathrm{~GB}$ of RAM).

On most of the protocols the execution takes only a few seconds. To generate larger interesting examples, we tested systems consisting of the parallel composition of two protocols, e.g., Running Example $\times 2$ is the parallel composition of two instances of the running example. Graphical representations of these protocols are in [?]. Observe that in general the size of the constructed global graph (i.e., the number of vertices) is significantly smaller than the size of $T S(S)$, see Running Example $\times 2$ for instance. We note that it is slightly more expensive to check the Running Example and the Logistic protocols. This is due to the fact that each of these protocols features at least one participant for which checking condition 2c) of Definition 3.5 is not trivial, because they receive information about a choice from different participants, e.g., Carol in $S_{r e}$. On the other hand, checking the Alternating 3-bit protocol is more time consuming due to larger $\bullet$-equivalence classes.

\section{Related Work}

Session Types In the context of multiparty session types, [27] first suggested a construction of a global protocol from a set of local session types, up to asynchronous sub-typing. A typing system which infers a global type [23] from a set of session types is given in [24]. Recursive constructions are restricted in this work, due to an inherently syntax-driven typing system, and multi-threaded participants are not supported (i.e., in terms of CFSMs, this means that mixed states are not allowed).

Example 6.1. Consider the GMC system of three machines below. Machine $s$ chooses to either continue interacting with machine $r$ (sending cont), or notify $\mathrm{r}$ that it wants to terminate (sending end), before collecting some information from machine $\mathrm{p}$ (collect).
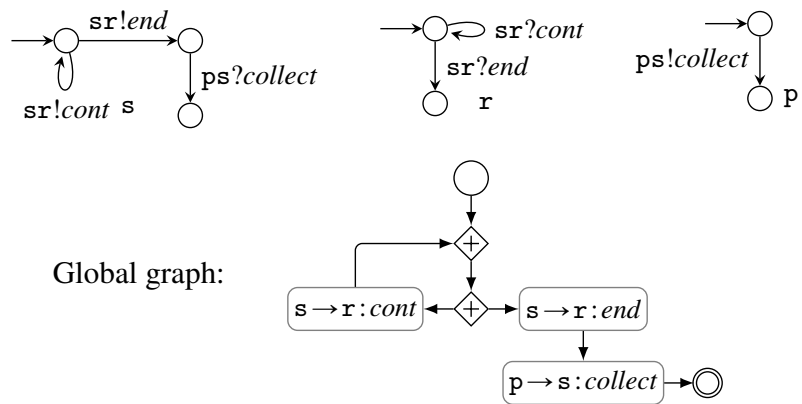

This system is not accepted by the typing system in [24] because machine $\mathrm{p}$ is not involved in the recursion (cf. rules $[\mu]$ and $[\mathbf{x}]$ in [24]).

In [20], the authors study the synthesis of global types from basic CFSMs, that is deterministic, non-mixed (each state is either sending or receiving), and directed (for each state, its outgoing transitions are all labelled by an action sending to, or receiving from, the same participant). Basic CFSMs do not allow to model general concurrency at the local level, since a machine cannot have mixed states. Note that machines A, B, and C in Figure 1 are not directed. The present work covers a much larger set of global protocols than [20, 24, 27]: we support mixed and non-directed states (hence, multi-threaded participants are allowed), recursive protocols are no longer restricted by a syntax oriented formalism, and explicit fork/join control points may be constructed.

The first translation from generalised global types into CFSMs was given in [19], where only sound properties were presented. The generalised global types of [19] are strictly included in GMC systems (Definition 3.6). The complete characterisation of global graphs and a construction algorithm were left as open problems. This paper solves these problems.

Choreographies Other recent works [9, 11, 15, 22] study the relationship between global and local specifications, but do not consider the problem of building global specifications from local ones. Namely, in [10], synchronisable systems are shown to preserve some reachability properties regardless the communication being asynchronous or synchronous. Essentially, systems are synchronisable if their synchronous behaviour is equivalent to their onebounded asynchronous behaviour (considering send actions only). In [11], the authors tackle the problem of determining whether a choreography is realisable. Essentially, a choreography is realisable if "it is possible to build a distributed system that communicates exactly as the choreography specifies". Choreographies in their work take the form of conversation protocols, that are finite state machines specifying the allowable sequence of interactions. A conversation protocol is akin to a global graph but without explicit construct for concurrent interactions, i.e., concurrent interactions must be specified by interleaving them. 
We observe that both synchronisability and realisability conditions require strong properties on message ordering. In comparison, the GMC condition requires $(i)$ the existence of a synchronous execution that encompasses all paths in each machine, and (ii) that each machine is either made aware every time a choice occurs or is not involved in the choice. In addition, a subtle difference between our machines and the machines in [10, 11] is that each of the latter machines has a unique buffer from which it can receive messages. Namely, their model is not suitable to reason about a CS as the interleaving of several multiparty sessions (where each participant has different receiving buffers in each session). In particular, their model cannot be used to represent programs which communicate via point-to-point communications, such as TCP connections between pairs of participants. We discuss a few examples that illustrate the main differences between the two communication models.

Example 6.2. Consider the GMC system below.
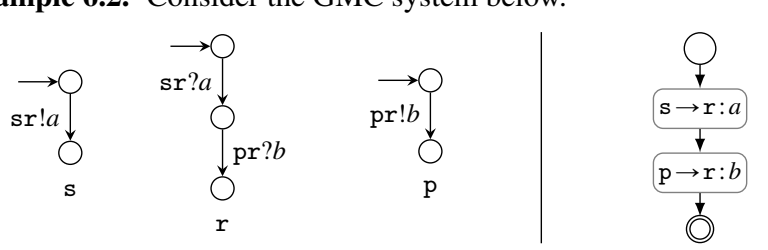

In our model, machine $r$ receives messages from $\mathrm{s}$ and $\mathrm{p}$ from two different buffers; therefore, this system is safe (since machine $r$ is always able to read message $a$ and then consume $b$ regardless of the order in which the messages from $\mathrm{s}$ and $\mathrm{p}$ arrive). In a model where machine $r$ has only one FIFO buffer to receive both messages from $s$ and $p$, machine $r$ will end up in an unspecified reception configuration if message $b$ reaches the queue before $a$. The system above is not synchronisable, since its synchronous execution differs from its one-bounded asynchronous execution (considering send actions only). Symmetrically, its choreography is not realisable.

Observe that the system $\left(A, B, C_{1}\right)$, from Example 3.3 is unsafe in our communication model, but safe in theirs (where it is synchronisable). In that model, safety follows from the fact that machine $\mathrm{B}$ would have only one buffer. Hence, if A chooses the left-hand side branch, message $a$ will be in B's queue before, thus B must execute its left-hand side branch; while if A chooses the right-hand side branch, $d$ will appear on B's queue first and the latter will then execute its right-hand side branch. Finally, note that the GMC system (resp. choreography) in Example 6.1 is not synchronisable (resp. realisable) either due to the "race" between the send actions from machines $\mathrm{s}$ and $\mathrm{p}$.

Automata \& MSC The term synthesis of CFSMs has been used to describe the reduction of CS to a more manageable (and decidable) model, e.g., with partial order approaches (see [29] for a summary of recent results). The acceptation of the term synthesis in this context is to identify a system of CFSMs that realises a protocol described by an incomplete specification (such as in [8, 28]). These approaches do not yield a global specification as instead achieved by our algorithm. In addition, our approach enables the verification of trace-based properties surveyed in [29]. For instance, the closed synthesis of CFSMs can be reduced to the construction from a regular language $L$ of a machine satisfying certain conditions related to buffer boundedness, deadlock-freedom, and words swapping.

In [26] a tool chain is given to synthesise an orchestrator (i.e., a message forwarder) from a set of finite-state machines communicating synchronously. This is transformed into a BPMN diagram via a Petri net transformation based on [18]. The work [31] gives an algorithm to compose several services. Each service is presented as an automaton and a set of automata are composed by a parallel product. The composite automaton is then transformed into a Petri net, using [18]. In both works, no result regarding safety or preservation of the behaviour of the original machines is given.

The work [6] studies whether Message Sequence Charts (MSC) imply unspecified scenarios (where MSCs are implemented by concurrent automata, but do not necessarily feature order-preserving communications). It gives conditions on MSCs for their implementation to be deadlock-free and realisable. MSCs are realisable if no other MSC may be inferable from them. It does not attempt to give an exhaustive global view of a distributed system, but focuses on identifying its possible misbehaviours.

\section{Conclusions \& Future Work}

We have given a complete algorithm whereby one can build a global graph (choreography) from any generalised multiparty compatible (GMC) system. GMC systems form a new class of communicating systems, and we have proved that any system in this class is safe and there exist efficient algorithms to check GMC. Our work effectively uses the theory of regions [18], bridging a gap between a set of distributed uncontrolled behaviours (represented by CFSMs) and well-structured graphical session types, while offering a scalable implementation for our framework.

Since the original machines can be recovered by projecting the constructed global graph (by Theorem 4.1, we can use our framework to develop a software development life cycle based on choreographies: a specification written as a choreography is projected onto a set of local models which will then be refined against their implementations. Such an approach can also be used to reverse-engineer existing distributed systems. We are currently collaborating with the Zero Deviation Lifecyle project [5] which proposes a platform to attain "near-zero defect leakage across the various phases of the software development lifecycle". Updating global scenarios against local models plays an important role in different stages of software life cycle in this architecture. Our framework applies naturally to this platform, which notably uses BPMN 2.0 Choreography [1] specifications and tools.

We also plan to investigate a relaxed version of the GMC condition which would allow to build global graphs whose projections are equivalent to the original system, up-to asynchronous orderpreserving communication [27].

\section{References}

[1] Business Process Model and Notation. http: //www.bpmn.org

[2] GMC-Synthesis. https://bitbucket.org/julien-lange/ gmc-synthesis

[3] Petrify. http://www.lsi.upc.edu/ jordicf/petrify/

[4] SAVARA Testable Architecture. http://www. jboss.org/savara

[5] Zero Deviation Lifecycle. http://www.zdlc.co

[6] R. Alur, K. Etessami, and M. Yannakakis. Inference of Message Sequence Charts. IEEE Trans. Software Eng., 29(7):623-633, 2003.

[7] E. Badouel and P. Darondeau. Theory of regions. In Petri Nets, volume 1491 of $L N C S$, pages 529-586. Springer, 1996.

[8] C. Baier, J. Klein, and S. Klüppelholz. Synthesis of reo connectors for strategies and controllers. Fundam. Inform., 130(1):1-20, 2014.

[9] S. Basu and T. Bultan. Choreography conformance via synchronizability. In $W W W$, pages 795-804. ACM, 2011.

[10] S. Basu, T. Bultan, and M. Ouederni. Synchronizability for verification of asynchronously communicating systems. In VMCAI, volume 7148 of LNCS. Springer, 2012.

[11] S. Basu, T. Bultan, and M. Ouederni. Deciding choreography realizability. In POPL, pages 191-202. ACM, 2012.

[12] F. Bonchi and D. Pous. Checking nfa equivalence with bisimulations up to congruence. In POPL, pages 457-468. ACM, 2013. 
[13] D. Brand and P. Zafiropulo. On communicating finite-state machines. JACM, 30(2):323-342, 1983.

[14] A. Bucchiarone, H. Melgratti, and F. Severoni. Testing service composition. In Proceedings of the 8th Argentine Symposium on Software Engineering (ASSE07), 2007.

[15] G. Castagna, M. Dezani-Ciancaglini, and L. Padovani. On global types and multi-party session. LMCS, 8(1), 2012.

[16] G. Cécé and A. Finkel. Verification of programs with half-duplex communication. I\&C, 202(2):166-190, 2005.

[17] W. W. W. Consortium. Web services choreography description language version 1.0. http://www.w3.org/TR/ws-cdl-10/ 2005.

[18] J. Cortadella, M. Kishinevsky, L. Lavagno, and A. Yakovlev. Deriving Petri Nets for Finite Transition Systems. IEEE Trans. Computers, 47 (8):859-882, 1998.

[19] P. Deniélou and N. Yoshida. Multiparty session types meet communicating automata. In ESOP, volume 7211 of $L N C S$, pages 194-213. Springer, 2012.

[20] P.-M. Deniélou and N. Yoshida. Multiparty compatibility in communicating automata: Characterisation and synthesis of global session types. In ICALP, volume 7966 of $L N C S$, pages 174-186, 2013.

[21] M. Güdemann, G. Salaün, and M. Ouederni. Counterexample guided synthesis of monitors for realizability enforcement. In ATVA, volume 7561 of $L N C S$, pages 238-253, 2012.

[22] S. Hallé and T. Bultan. Realizability analysis for message-based interactions using shared-state projections. In SIGSOFT FSE, pages 27-36. ACM, 2010.

[23] K. Honda, N. Yoshida, and M. Carbone. Multiparty asynchronous session types. In POPL, pages 273-284. ACM, 2008.

[24] J. Lange and E. Tuosto. Synthesising Choreographies from Local Session Types. In CONCUR, volume 7454 of $L N C S$, pages 225-239. Springer, 2012.

[25] R. Lanotte, A. Maggiolo-Schettini, and A. Troina. Weak bisimulation for probabilistic timed automata. Theor. Comput. Sci., 411(50):42914322, 2010.

[26] S. McIlvenna, M. Dumas, and M. T. Wynn. Synthesis of orchestrators from service choreographies. In APCCM, volume 96 of CRPIT, pages 129-138. ACS, 2009.

[27] D. Mostrous, N. Yoshida, and K. Honda. Global principal typing in partially commutative asynchronous sessions. In ESOP, volume 5502 of LNCS, pages 316-332. Springer, 2009.

[28] M. Mukund, K. N. Kumar, and M. A. Sohoni. Synthesizing distributed finite-state systems from MSCs. In CONCUR, volume 1877 of LNCS, pages 521-535. Springer, 2000.

[29] A. Muscholl. Analysis of communicating automata. In LATA, volume 6031 of $L N C S$, pages 50-57. Springer, 2010.

[30] G. Salaün, L. Bordeaux, and M. Schaerf. Describing and reasoning on web services using process algebra. In ICWS, pages 43-. IEEE Computer Society, 2004.

[31] Y. Wang, A. Nazeem, and R. Swaminathan. On the optimal Petri net representation for service composition. In ICWS, pages 235-242. IEEE Computer Society, 2011.

[32] D. M. Yellin and R. E. Strom. Protocol specifications and component adaptors. ACM Trans. Program. Lang. Syst., 19(2):292-333, 1997.

\section{A. Equivalences between Petri Nets}

We give the formal definitions of the reachability graph of a Petri net and weak-bisimulation, which are used in Section 4

Definition A.1 (Reachability graph [18]). Given $\mathbb{N}=\left(P, T, F, m_{0}\right)$, we say that a transition $t \in T$ is enabled at marking $m_{1}$ if all its input places are marked. An enabled transition $t$ may fire, producing a new marking $m_{2}$ with one less token in each input place and one more token in each output place. We write $m_{1} \stackrel{t}{\rightarrow} m_{2}$, if $m_{2}$ is reachable from $m_{1}$ by firing $t$, and write $\rightarrow^{*}$ for the reflexive transitive closure of $\rightarrow$.

The reachability graph of $\mathbb{N}$ is the transition system $R G(\mathbb{N})=$ $\left(M, m_{0}, \hat{E}, \rightarrow\right)$ such that $M=\left\{m \mid m_{0} \rightarrow^{*} m\right\}$;

- $\rightarrow=\left\{\left(m_{1}, l a b(t), m_{2}\right) \mid m_{1}, m_{2} \in M \wedge m_{1} \stackrel{t}{\rightarrow} m_{2}\right\}$ (where $l a b(t)=$ $\varepsilon$ if the label of $t$ is $\varepsilon$, and return the label $e$ of $t$ otherwise); and

- $\hat{E}=\left\{e \mid \exists\left(m_{1}, e, m_{2}\right) \in \rightarrow \wedge \neq \varepsilon\right\}$;

Let $m \stackrel{e}{\rightarrow} m^{\prime}$ iff $\left(m, e, m^{\prime}\right) \in \rightarrow$ and $m \stackrel{e}{\Rightarrow} m^{\prime}$ iff $m(\stackrel{\varepsilon}{\rightarrow})^{*} \stackrel{e}{\rightarrow}(\stackrel{\varepsilon}{\rightarrow})^{*} m^{\prime}$, with $e \neq \varepsilon$.

The notion of weak bisimulation between two transition systems is given in Definition A.2 (adapted from [25])

Definition A.2 (Weak bisimulation). Let $T=\left(M, m_{0}, \hat{E}, \rightarrow\right)$ be a transition system. A weak bisimulation on $T$ is an equivalence relation $\mathcal{B} \subseteq M \times M$ s.t. for all $\left(m_{1}, m_{2}\right) \in \mathcal{B}$, the following holds

- $m_{1} \stackrel{e}{\rightarrow} m_{1}^{\prime}$ implies that there is $m_{2}^{\prime}$ such that $m_{2} \stackrel{e}{\Rightarrow} m_{2}^{\prime}$ and $\left(m_{1}^{\prime}, m_{2}^{\prime}\right) \in \mathcal{B}$; and

- $m_{2} \stackrel{e}{\rightarrow} m_{2}^{\prime}$ implies that there is $m_{1}^{\prime}$ such that $m_{1} \stackrel{e}{\Rightarrow} m_{1}^{\prime}$ and $\left(m_{1}^{\prime}, m_{2}^{\prime}\right) \in \mathcal{B}$.

Two states $m_{1}$ and $m_{2}$ are called weakly bisimilar on $T$, written $m_{1} \approx_{T} m_{2}$, iff $\left(m_{1}, m_{2}\right) \in \mathcal{B}$ for some weak bisimulation $\mathcal{B}$.

Two transition systems $T_{i}=\left(M_{i}, m_{0}^{i}, \hat{E}_{i}, \rightarrow_{i}\right), i \in\{1,2\}$, such that $M_{1} \cap M_{2}=\varnothing$, are weakly bisimilar, written $T_{1} \approx T_{2}$, if given

- $M^{\prime}=M_{1} \cup M_{2} \cup\left\{m_{0}\right\}$ and $\hat{E}^{\prime}=\hat{E}_{1} \cup \hat{E}_{2}$,

- $T^{\prime}=\left(M^{\prime}, m_{0}, \hat{E}^{\prime}, \rightarrow_{1} \cup \rightarrow_{2} \cup\left\{\left(m_{0}, \varepsilon, m_{0}^{1}\right),\left(m_{0}, \varepsilon, m_{0}^{2}\right)\right\}\right)$

$m_{0}^{1} \approx T^{\prime} m_{0}^{2}$ holds.

\section{B. From Petri Nets to Global Graphs}

In this section we give the detailed transformations omitted in Section 4 The algorithm to construct a global graph $G$ from a synchronous transition system $T S(S)$ consists of the following steps: (1) using the algorithm of Cortadella et al. [18], we derive a Petri net $\mathbb{N}$ from $T S(S)$; (2) we transform $\mathbb{N}$ so that its initial marking consists of exactly one place (Transformation B.1 below); (3) we join transitions whenever possible, so to make joins and forks explicit (Transformation B.2 below); (4) we transform the net of (3) into a pre-global graph (Transformation B.3 below); finally, we "clean-up" the pre-global graph of any unnecessary vertexes so to obtain a global graph (Transformation B.4 below).

Definition B.1 (Labelled net). A labelled Petri net, or net, $\mathbb{N}$ is a quadruple $\left(P, T, F, m_{0}\right)$ with $P$ a set of places (ranged over by $\left.p\right), T$ a set of transitions (ranged over by $t), F \subseteq(P \times T) \cup(T \times P)$ the flow relation, and $m_{0}$ the initial marking. Each transition $t \in T$ is labelled with an event $e \in \hat{E}$, or marker $\varepsilon$ (the latter representing a silent transition). We let $x$ range over elements of $P \cup T$. As usual, $\bullet$ (resp. $x^{\bullet}$ ) is the preset (resp. postset) of $x$. A net is called safe if, for all reachable markings, no more than one token can appear in each place; in which case the reachable markings (including $m_{0}$ ) are sets of places. A net is extended free-choice if $\forall p \in P, \forall t \in T:(p, t) \in F \Longrightarrow\left(\bullet \times p^{\bullet}\right) \subseteq F$.

In the second step (2), we transform a Petri net obtained from Cortadella's algorithm into a Petri net whose initial marking consists of exactly one place. This allows us to construct a global graph that has a unique starting point (source).

Transformation B.1 (One-source net). Given a labelled Petri net $\mathbb{N}=\left(P, T, F, m_{0}\right)$, the one-source net of $\mathbb{N}$ is $\mathbb{N}^{\prime}=\left(P \cup\left\{p_{0}\right\}, T \cup\right.$ $\left.\left\{t^{\prime}\right\}, F^{\prime},\left\{p_{0}\right\}\right)$ such that $p_{0} \notin P, t^{\prime} \notin T$ is labelled by $\varepsilon$, and $F^{\prime}=$ $F \cup\left\{\left(p_{0}, t^{\prime}\right)\right\} \cup \bigcup_{p \in m_{0}}\left\{\left(t^{\prime}, p\right)\right\}$. 
Proposition B.1. Transformation B.1 is computable in linear time in the size of $m_{0}$.

We can now state the following result, formalising the soundness of Transformation B.1.

Lemma B.1. If $T$ is the reachability graph of the Petri net $\mathbb{N}$ obtained from $T S(S)$ via the algorithm in [18], and $T^{\prime}$ is the reachability graph of the Petri net obtained after applying Transformation B.1, then $T \approx T^{\prime}$.

Next, Transformation B.2 ensures that parallel gates are used "as much as possible" in the graph (instead of mixing choice and parallel gates). In fact, Transformation B.2 joins sets of places having the same preset or postset to decrease the number of choice gates.

Transformation B.2 (Joined net). The joined net of $\mathbb{N}=\left(P, T, F, m_{0}\right)$ is a net $\mathbb{N}^{\prime}=\left(P^{\prime}, T^{\prime}, F^{\prime}, m_{0}\right)$ such that the following transformations are applied repeatedly:

1. for all maximal $X \subseteq P$ s.t. $|X|>1$ and $\forall p_{1}, p_{2} \in X:{ }^{\bullet} p_{1}=$ - $p_{2} \wedge\left|{ }^{\bullet} p_{1}\right|>1, P^{\prime}=P \cup\left\{p^{\prime}\right\}$ and $T^{\prime}=T \cup\left\{t^{\prime}\right\}$ with $p^{\prime} \notin P$ and $t^{\prime} \notin T$ and labelled by $\varepsilon$; also, chosen $p \in X, F^{\prime}=\left\{\left(p^{\prime}, t^{\prime}\right)\right\} \cup$ $\left(\bullet p \times\left\{p^{\prime}\right\}\right) \cup\left(\left\{t^{\prime}\right\} \times X\right) \cup F \backslash \bigcup_{x \in X} \bullet x \times\{x\}$

2. for all maximal $X \subseteq P$ s.t. $|X|>1$ and $\forall p_{1}, p_{2} \in X: p_{1}^{\bullet}=$ $p_{2} \bullet \wedge\left|p_{1} \bullet\right|>1, P^{\prime}=P \cup\left\{p^{\prime}\right\}$ and $T^{\prime}=T \cup\left\{t^{\prime}\right\}$ with $p^{\prime} \notin$ $P$ and $t^{\prime} \notin T$ and labelled by $\varepsilon$; also, chosen $p \in X, F^{\prime}=$ $\left\{\left(t^{\prime}, p^{\prime}\right)\right\} \cup\left(\left\{p^{\prime}\right\} \times p^{\bullet}\right) \cup\left(X \times\left\{t^{\prime}\right\}\right) \cup F \backslash \bigcup_{x \in X}\{x\} \times x^{\bullet}$.

Note that the definition of $F^{\prime}$ does not depend on the choice of $p$.

Proposition B.2. Transformation B.2 is computable in polynomial time in the size of $\mathbb{N}$.

Since we are working with safe nets, we have the result below.

Lemma B.2. If $T$ (resp. $T^{\prime}$ ) is the reachability graph of the Petri net $\mathbb{N}$ obtained after Transformation B.1 (resp. Transformation B.2, then $T \approx T^{\prime}$.

Definition B.2 (Graph composition). Let $\mathbb{N}_{i}=\left(P_{i}, T_{i}, F_{i}, m_{0_{i}}\right)$ with $i \in\{1,2\}$ be two nets and $G_{i}=\left\langle V_{i}, A_{i}, \Lambda_{i}\right\rangle$ two graphs such that $V_{i}=P_{i} \cup T_{i} \cup F_{i}, i \in\{1,2\}$, the composition of $G_{1}$ and $G_{2}$, denoted by $G_{1} \uplus G_{2}$ is a graph $\langle V, A, \Lambda\rangle$ defined as:

$$
\begin{aligned}
\cdot & V=\left\{v \in V_{1} \mid v \in F_{1} \Longrightarrow v \notin V_{2}\right\} \cup\left\{v \in V_{2} \mid v \in F_{2} \Longrightarrow v \notin V_{1}\right\} \\
\cdot & A=\left(\left(A_{1} \cup A_{2}\right) \cap V \times V\right) \cup\left\{\left(v, v^{\prime}\right) \mid \exists v^{\prime \prime} \in F_{i}:\left(v, v^{\prime \prime}\right) \in A_{i},\left(v^{\prime \prime}, v^{\prime}\right) \in\right. \\
& \left.A_{j} \wedge i \neq j \in\{1,2\}\right\}
\end{aligned}
$$

Intuitively, the composition of the graphs consists of (1) the union of the two sets of vertexes, except flow elements $(p, t)$ and $(t, p)$ if they appear in both $V_{1}$ and $V_{2}$; and (2) the union of the two sets of arcs between vertexes in $V$, and each pair of arcs of the form $\left(v,\left(x, x^{\prime}\right)\right)$ or $\left(\left(x, x^{\prime}\right), v^{\prime}\right)$ is replaced by a single arc $\left(v, v^{\prime}\right)$.

Transformation B.3 (Pre-global graph). The pre-global graph of $\mathbb{N}=\left(P, T, F,\left\{p_{0}\right\}\right)$ is a tuple $\langle V, A, \Lambda\rangle$ such that $V=P \cup T \cup F, \Lambda$ is a labelling function such that $\Lambda(v)=v$ if $v \in P \cup F$ or $v \in T$ labelled by $\varepsilon$, and $\Lambda(v) \in \hat{E} \cup\{\odot, \bigcirc, \uparrow, \square\}$ otherwise; and $A$ is given by:

$$
T_{g}(\mathbb{N})=\biguplus_{x \in P \cup T} T_{i}(x) \uplus T_{o}(x) \quad \text { where, given } x \in P \cup T:
$$

$$
\begin{aligned}
& T_{i}(x) \stackrel{\text { def }}{=} \begin{cases}\bigcirc \rightarrow x & \text { if } \bullet x=\varnothing \\
\left(x^{\prime}, x\right) \rightarrow x & \text { if } \bullet x=\left\{x^{\prime}\right\} \\
\left(x_{1}, x\right) \neg & \\
\vdots \\
\left(x_{i}, x\right) \rightarrow \underset{\otimes}{\vdots} \rightarrow x & \text { if } \bullet x=\left\{x_{1}, \ldots, x_{k}\right\} \\
\left(x_{k}, x\right) \longrightarrow & \end{cases} \\
& T_{o}(x) \stackrel{\text { def }}{=} \begin{cases}\begin{array}{ll}
x \rightarrow & \text { if } x^{\bullet}=\varnothing \\
x \rightarrow\left(x, x^{\prime}\right) & \text { if } x^{\bullet}=\left\{x^{\prime}\right\}
\end{array} \\
\longrightarrow\left[\begin{array}{cc}
\left(x, x_{1}\right) \\
\vdots \\
x \rightarrow\left(x, x_{i}\right) \\
\vdots \\
\longrightarrow\left(x, x_{k}\right)
\end{array}\right. & \text { if } x^{\bullet}=\left\{x_{1}, \ldots, x_{k}\right\}\end{cases}
\end{aligned}
$$

with $k>1, \otimes=\diamond$ if $x \in P$, and $\otimes=\square$ if $x \in T$.

The pre-global graph of $S_{r e}$ (Figure 1) is given in Figure 5 (right). Observe that all the vertexes of the form $\left(x, x^{\prime}\right)$, corresponding to an element of the flow relation, are removed as part of the graph composition (Definition B.2).

Proposition B.3. Transformation B.3 is computable in polynomial time in the size of $\mathbb{N}$.

We define the final transformation which cleans up a pre-global graph by removing unnecessary vertexes and arcs.

Transformation B.4. A global graph $G=\langle V, A, \Lambda\rangle$ is obtained from a pre-global graph $\left\langle P \cup T \cup F, A^{\prime}, \Lambda^{\prime}\right\rangle$ by applying the following transformation: (1) replace each pair of transition $(x, p),\left(p, x^{\prime}\right) \in$ $A^{\prime}$ by $\left(x, x^{\prime}\right) \in A$; (2) replace each pair of transition $(x, t),\left(t, x^{\prime}\right) \in A^{\prime}$, with $t$ labelled by $\varepsilon$, by $\left(x, x^{\prime}\right) \in A$; and (3) label each $t$ which is labelled by $\left(q_{\mathrm{s}}, q_{\mathrm{r}}, \mathrm{s} \rightarrow \mathrm{r}: a\right)$ in $\mathbb{N}$, by $\mathrm{s} \rightarrow \mathrm{r}: a$.

Proposition B.4. Transformation B.4 is computable in polynomial time in the size of $\mathbb{N}$.

\section{Projections of Global Graphs}

The definition of the projection of a global graph onto a participant, used in Section 4 is given below. We first define a parallel composition of automata, which is required to project global graphs with a participant appearing in different threads. We define the ${ }_{-}^{\star}$ function on vector of states:

$$
\vec{q}^{\star} \stackrel{\text { def }}{=} \begin{cases}\vec{q}_{1} & \text { if } \vec{q}=\left(\vec{q}_{1}, \vec{q}_{2}\right) \text { and } \vec{q}_{1}^{\star}=\vec{q}_{2}^{\star} \\ \vec{q} & \text { otherwise }\end{cases}
$$

we overload it on sets of vector of states, i.e., $Q^{\star} \stackrel{\text { def }}{=}\left\{\vec{q}^{\star} \mid \vec{q} \in Q\right\}$. We define:

$$
q \Subset \vec{q}^{\prime} \Longleftrightarrow\left\{\begin{array}{l}
q=q^{\prime \prime} \text { and } \vec{q}^{\prime}=\left(q^{\prime \prime}\right), \text { or } \\
\vec{q}^{\prime}=\left(\vec{q}_{1}, \vec{q}_{2}\right) \wedge q \Subset \vec{q}_{i} \wedge i \in\{1,2\}
\end{array}\right.
$$

We write $q \Subset \vec{q}^{\prime}$ iff $\neg\left(q \Subset \vec{q}^{\prime}\right)$ and we overload the operator $\Subset$ on set of states such that $q \Subset Q \Longleftrightarrow \exists \vec{q}^{\prime} \in Q: q \Subset \vec{q}^{\prime}$.

Definition C.1 (Parallel composition). The composition of $M_{\mathrm{i}}=$ $\left(Q_{\dot{\mathrm{i}}}, q_{0}^{\mathrm{i}}, \delta_{\mathrm{i}}\right), \mathrm{i} \in\{1,2\}$, written $M_{1} \| M_{2}$, is the automaton $\left(\left(Q_{1} \times\right.\right.$ $\left.\left.Q_{2}\right)^{\star},\left(q_{0}^{1}, q_{0}^{2}\right)^{\star}, \delta\right)$ s.t.

$$
\begin{aligned}
& \left(\left(q_{1}, q_{2}\right)^{\star}, \ell,\left(q_{1}^{\prime}, q_{2}^{\prime}\right)^{\star}\right) \in \delta \\
& \Longleftrightarrow \begin{cases}\left(q_{\mathrm{i}}, \ell, q_{\mathrm{i}}^{\prime}\right) \in \delta_{\mathrm{i}} & \text { if } q_{\mathrm{j}}=q_{\mathrm{j}}^{\prime}, q_{\mathrm{i}} \Subset Q_{\mathrm{j}} \text { and } \mathrm{i} \neq \mathrm{j} \in\{1,2\} \\
\left(q_{\mathrm{i}}, \ell, q_{\mathrm{i}}^{\prime}\right) \in \delta_{\mathrm{i}} & \text { if } q_{\mathrm{i}} \Subset Q_{\mathrm{j}} \text { and } \mathrm{i} \neq \mathrm{j} \in\{1,2\}\end{cases}
\end{aligned}
$$

Notice that $\|$ is a commutative and associative operation. Below, we give the definition of the projection function.

Definition C.2 (Projection). Given $G=\left\langle V^{\prime}, A, \Lambda\right\rangle$ and $v \in V^{\prime}$, the projection of $(G, v, V)$ onto $\mathrm{p}$, denoted by $\left.(G, v, V)\right|_{\mathrm{p}}$, is defined as 
follows:

$$
\begin{aligned}
& (G, v, V) l_{\mathrm{p}}= \\
& \begin{cases}\langle\{v\}, v, \varnothing\rangle & \text { if } \Lambda(v)=\text { ○ or } v \in V \\
\left\langle Q \cup\{v\}, v, \delta \cup\left\{\left(v, \ell, v^{\prime}\right)\right\}\right\rangle & \text { if } v^{\prime} \in v^{\bullet}, \ell=\mathrm{s} \rightarrow \mathrm{r}: a l_{\mathrm{p}} \\
& \text { and }\left\langle Q, v^{\prime}, \delta\right\rangle=\left(G, v^{\prime}, V \cup\{v\}\right) l_{\mathrm{p}} \\
\left\langle\{v\} \cup \bigcup_{v^{\prime} \in v^{\bullet}} Q_{v^{\prime}}, v, \bigcup_{v^{\prime} \in v^{\bullet}} \delta_{v^{\prime}} \cup\left(v, \varepsilon, v^{\prime}\right)\right\rangle \quad \text { if } \Lambda(v) \in\{O, \uparrow\} & \text { and }\left\langle Q_{v^{\prime}}, v^{\prime}, \delta_{v^{\prime}}\right\rangle=\left(G, v^{\prime}, V \cup\{v\}\right) l_{\mathrm{p}} \\
\left\langle Q, v, \delta \cup\left(v, \varepsilon, v^{\prime \prime}\right)\right\rangle & \text { if } \Lambda(v)=\square \\
& \text { and }\left\langle Q, v^{\prime \prime}, \delta\right\rangle=\|_{v^{\prime} \in v^{\bullet}}\left(G, v^{\prime}, V \cup\{v\}\right) l_{\mathrm{p}}\end{cases}
\end{aligned}
$$

Given a vertex $v \in V^{\prime}$ such that $\Lambda(v)=0$, the projection of $G$ onto p, written $G l_{\mathrm{p}}$, is the automaton, minimised wrt. language equivalence, $\left(Q, q_{0}, \delta, \mathbb{A}\right)$ with $(G, v, \varnothing) \iota_{\mathrm{p}}=\left\langle Q, q_{0}, \delta\right\rangle$.

The projection of a global graph onto $\mathrm{p}$ uses the auxiliary function $\left.(G, v, V)\right|_{\mathrm{p}}$. The function takes the following parameter, $\mathrm{p}$ : the identifier of the participant onto the projection is invoked, $G$ : the global graph to be projected, $v$ : a node in $G$ used as an initial node for the projection, and $V$ : a set of visited nodes.

If $v$ has already been visited or it is a sink node, then a single state automaton is returned. If $v$ is labelled by $\mathrm{s} \rightarrow \mathrm{r}: a$, then the projection of it successor is connected to $v$ by a transition labelled by either $\operatorname{pr} ! a, \operatorname{sp} ? a$, or $\varepsilon$. If $v$ is a choice gate or the source node, then the projection of each successor of $v$, connected by an $\varepsilon$ transition from $v$, is returned. If $v$ is a parallel gate, then the parallel composition of the projections of its successors are returned, connected to $v$ by an $\varepsilon$ transition. The parallel composition of automata uses Definition C.3 so that state identities are normalised and visited nodes are comparable with nodes produced by composing automata. 


\section{Proofs of Section 3}

In this section, we first prove the key properties of the relations defined in the paper. Then we prove the main theorem (soundness), the reachability lemma and complexity results stated in Section 3 . We use the following functions in the proofs:

$$
\begin{aligned}
& \text { 1. } \operatorname{snd}\left(\left(q_{\mathrm{s}}, q_{\mathrm{r}}, \mathrm{s} \rightarrow \mathrm{r}: a\right)\right)=\operatorname{snd}(\mathrm{s} \rightarrow \mathrm{r}: a) \stackrel{\text { def }}{=} \mathrm{s} ; \\
& \text { 2. } \operatorname{rcv}\left(\left(q_{\mathrm{s}}, q_{\mathrm{r}}, \mathrm{s} \rightarrow \mathrm{r}: a\right)\right)=\mathrm{rcv}(\mathrm{s} \rightarrow \mathrm{r}: a) \stackrel{\text { def }}{=} \mathrm{r} ; \text { and } \\
& \text { 3. } \operatorname{id}\left(\left(q_{\mathrm{s}}, q_{\mathrm{r}}, \mathrm{s} \rightarrow \mathrm{r}: a\right)\right)=\operatorname{id}(\mathrm{s} \rightarrow \mathrm{r}: a) \stackrel{\text { def }}{=}\{\mathrm{s}, \mathrm{r}\} \text {. }
\end{aligned}
$$

\section{D.1 Properties of $T S(S)$}

Lemma D.1. Let $S=\left(M_{\mathrm{p}}\right)_{\mathrm{p} \in \mathcal{P}}$ be a GMC system and $T S(S)=$ $\left(N, n_{0}, \hat{E}, \Longrightarrow\right)$, for all $\mathrm{p} \in \mathcal{P}$ and for all $q \in Q_{\mathrm{p}}$ such that $\left(q, \ell_{1}, q_{1}\right)$, $\left(q, \ell_{2}, q_{2}\right) \in \delta_{\mathrm{p}}$ for some $q_{1}, q_{2} \in Q_{\mathrm{p}}$, there is $n, n_{1}, n_{2} \in N$ such that $n[\mathrm{p}]=q$,

$$
\begin{aligned}
& \cdot n \stackrel{\pi_{1}}{\Rightarrow} n_{1} \stackrel{e_{1}}{\Rightarrow}, \text { with } \pi_{1} l_{\mathrm{p}}=\varepsilon, e_{1} l_{\mathrm{p}}=\ell_{1} ; \text { and } \\
& \text { - } n \stackrel{\pi_{2}}{\Rightarrow} n_{2} \stackrel{e_{2}}{\Rightarrow}, \text { with } \pi_{2} l_{\mathrm{p}}=\varepsilon, e_{2} l_{\mathrm{p}}=\ell_{2} \text {. }
\end{aligned}
$$

Proof. This result follows directly from the representability condition.

Lemma D.2. Let $S=\left(M_{\mathrm{p}}\right)_{\mathrm{p} \in \mathcal{P}}$ be a system and $T S(S)=\left(N, n_{0}, \hat{E}, \Longrightarrow\right.$ ). For all $n \neq n^{\prime} \in N$, if $n \stackrel{e}{\Rightarrow} n^{\prime}$, then $n^{\prime} \stackrel{e}{\risingdotseq}$.

Proof. By contradiction, assume there are $n \neq n^{\prime} \in N$ such that $n \stackrel{e}{\Longrightarrow} n^{\prime}$ and $n^{\prime} \stackrel{e}{\Longrightarrow} n^{\prime \prime}$, where $e=\left(q_{\mathrm{s}}, q_{\mathrm{r}}, \mathrm{s} \rightarrow \mathrm{r}: a\right)$. There are two cases depending on whether the events come from distinct events in $\hat{\delta}$.

1. Assume $\left(n, e, n^{\prime}\right),\left(n^{\prime}, e, n^{\prime \prime}\right) \in \hat{\delta}$, then it must be the case that $\left(q_{\mathrm{s}}, \mathrm{sr} ! a, q_{\mathrm{s}}\right) \in \delta_{\mathrm{s}}$ and $\left(q_{\mathrm{r}}, \mathrm{sr} ? a, q_{\mathrm{r}}\right) \in \delta_{\mathrm{r}}$, i.e., there is a corresponding self-loop in machines $s$ and $r$. Since the local source state and target states of both machines are the same, this contradicts the assumption that $n \neq n^{\prime}$.

2. Assume $\left(n, e, n^{\prime}\right),\left(n^{\prime}, e^{\prime}, n^{\prime \prime}\right) \in \hat{\delta}$, with $e \neq e^{\prime}, e^{\prime}=\left(q_{\mathrm{s}}^{\prime}, q_{\mathrm{r}}^{\prime}, \mathrm{s} \rightarrow\right.$ $\mathrm{r}: a)$, and $e \bowtie e^{\prime}$. We must have that $\delta_{\mathrm{s}}\left(q_{\mathrm{s}}, \mathrm{sr} ! a\right)=q_{\mathrm{s}}^{\prime}$ and $\delta_{\mathrm{r}}\left(q_{\mathrm{r}}, \mathrm{sr} ? a\right)=q_{\mathrm{r}}^{\prime}$; and since $e \neq e^{\prime}$ we must have that $q_{\mathrm{s}} \neq$ $q_{\mathrm{s}}^{\prime}$ or $q_{\mathrm{r}} \neq q_{\mathrm{r}}^{\prime}$. Take $q_{\mathrm{r}} \neq q_{\mathrm{r}}^{\prime}$, we must have $\left(q_{\mathrm{r}}\right.$, sr? $\left.a, q_{\mathrm{r}}^{\prime}\right)$, $\left(q_{\mathrm{r}}^{\prime}, \mathrm{sr} ? a, q_{\mathrm{r}}^{\prime \prime}\right) \in \delta_{\mathrm{r}}$. This implies that we have

$$
\neg\left(\left(q_{\mathrm{r}}, \mathrm{sr} ? a, q_{\mathrm{r}}^{\prime}\right) \bullet\left(q_{\mathrm{r}}^{\prime}, \mathrm{sr} ? a, q_{\mathrm{r}}^{\prime \prime}\right)\right)
$$

since $\operatorname{sr} ? a \in \operatorname{act}\left(q_{\mathrm{r}}, q_{\mathrm{r}}^{\prime}\right)$.

The cases where $q_{\mathrm{s}} \neq q_{\mathrm{s}}^{\prime}$ and $q_{\mathrm{s}} \neq q_{\mathrm{s}}^{\prime} \wedge q_{\mathrm{r}} \neq q_{\mathrm{r}}^{\prime}$ are similar. Hence, by Definition 3.1. we conclude that $\neg\left(e \bowtie e^{\prime}\right)$ which contradicts our hypothesis.

\section{D.2 Properties of $\prec$}

In this section, we fix $S=\left(M_{\mathrm{p}}\right)_{\mathrm{p} \in \mathcal{P}}$ and $T S(S)=\left(N, n_{0}, \hat{E}, \Longrightarrow\right)$.

Lemma D.3. Let $n, n^{\prime}, n^{\prime \prime} \in T S(S)$, if $n<n^{\prime}$ and $n^{\prime}<n^{\prime \prime}$, then $n<n^{\prime \prime}$.

Proof. Assume $n<n^{\prime}$ and $n^{\prime}<n^{\prime \prime}$, by definition of $\prec$, we have

$$
n_{0} \Longrightarrow^{*} n \Longrightarrow^{*} n^{\prime} \Longrightarrow^{*} n^{\prime \prime}
$$

which implies that there is a simple path from $n$ to $n^{\prime}$.

By contradiction, assume $\neg\left(n<n^{\prime \prime}\right)$. This implies that either (i) $n=n^{\prime \prime}$, which contradicts the fact that $n^{\prime} \prec n^{\prime \prime}$; or (ii) there is a simple path from $n_{0}$ to $n$ that includes $n^{\prime \prime}$, thus there is also a simple path from $n_{0}$ to $n^{\prime}$ that includes $n^{\prime \prime}$, which contradicts our assumption.
Lemma D.4. Let $n_{1} \ldots n_{k}$ be a simple path in $T S(S)$, such that $n_{k} \Longrightarrow n_{1}$. For all $1 \leqslant i, j \leqslant k$, if $n_{i}<n_{j}$, then $\neg\left(n_{j} \prec n_{i}\right)$.

Proof. Take $n_{i}$ and $n_{j}$ such that $n_{i}<n_{j}$. This means that $n_{j}$ never appears on a path from $n_{0}$ to $n_{i}$. Since $n_{1} \ldots n_{k}$ forms a cycle, we must have $n_{i} \Longrightarrow^{*} n_{j}$ and $n_{j} \Longrightarrow^{*} n_{i}$. Thus we have

$$
n_{0} \Longrightarrow^{*} n_{i}{ }^{*} n_{j} \rightleftharpoons^{*} n_{i}
$$

and there is a simple path from $n_{0}$ to $n_{j}$ that includes $n_{i}$ (cf. simple path assumption), which implies that we cannot have $n_{j} \prec n_{i}$.

D.3 Properties of $\ln \left(n, e_{1}, e_{2}\right)$

Lemma D.5. Let $S=\left(M_{\mathrm{p}}\right)_{\mathrm{p} \in \mathcal{P}}$ and $T S(S)=\left(N, n_{0}, \hat{E}, \Longrightarrow\right)$. For all $n \in N$, if $n \stackrel{e_{1}}{\Longrightarrow}, n \stackrel{e_{2}}{\Rightarrow}$, and $e_{1} \neq e_{2}$, then $\ln \left(n, e_{1}, e_{2}\right) \neq \varnothing$.

Proof. By assumption, there is at least one node that fires both $e_{1}$ and $e_{2}$ and is reachable from $n$, i.e., $n$ itself.

If $n$ is the only node from which $e_{1}$ and $e_{2}$ are fireable, $n \stackrel{e_{1}}{\Rightarrow} n_{1}$, and $n \stackrel{e_{2}}{\Longrightarrow} n_{2}$, then $\ln \left(n, e_{1}, e_{2}\right)=\left\{\left(n_{1}, n_{2}\right)\right\}$. Note that the results also holds if $n \Longrightarrow n$ since we have $\neg(n<n)$, by definition of $\prec$. If $n$ is not the only node from which $e_{1}$ and $e_{2}$ are fireable, then there are two cases:

1. None of these nodes is reachable from $n$, in which case the result is the same as above, i.e., $\ln \left(n, e_{1}, e_{2}\right)=\left\{\left(n_{1}, n_{2}\right)\right\}$.

2. Let $N_{0} \stackrel{\text { def }}{=}\left\{n^{\prime} \mid n \Longrightarrow^{*} n^{\prime} \wedge n^{\prime} \stackrel{e_{1}}{\Rightarrow} \wedge n^{\prime} \stackrel{e_{1}}{\Rightarrow}\right\}$, note that $n \in N_{0}$, and, by contradiction, assume $\ln \left(n, e_{1}, e_{2}\right)=\varnothing$. Hence, none of the nodes in $N_{0}$ satisfy the condition, i.e.,

$$
\forall n^{\prime} \in N_{0}: \exists n^{\prime \prime} \in N_{0}: n^{\prime} \Longrightarrow n^{\prime \prime} \wedge n^{\prime} \prec n^{\prime \prime}
$$

Since the number of nodes in $T S(S)$ is finite, we must have a cycle in $N_{0}$.

By Lemmas D.3 and D.4 we know that $<$ forms a strict partial order on the nodes of each simple cycle in $N_{0}$. Thus, for each simple cycle consisting of nodes in $N_{0}^{\prime} \subseteq N_{0}$, each top element $n^{\prime} \in N_{0}^{\prime}$ we have:

$$
\forall n^{\prime \prime} \in N_{0}^{\prime}: n^{\prime} \Longrightarrow n^{\prime \prime} \Longrightarrow \neg\left(n^{\prime}<n^{\prime \prime}\right)
$$

Let $N_{0}^{\prime \prime} \subseteq N_{0}$ be the set of all top elements of each simple cycle in $N_{0}$, none of the nodes in $N_{0}^{\prime}$ satisfy the condition only if

$$
\forall n^{\prime} \in N_{0}^{\prime \prime}: \exists n^{\prime \prime} \in N_{0}^{\prime \prime}: n^{\prime} \Longrightarrow n^{\prime \prime} \wedge n^{\prime} \prec n^{\prime \prime}
$$

We show that given $n_{i}, n_{j} \in N_{0}^{\prime \prime}$ such that $n_{i} \Longrightarrow n_{j}$ and $n_{i}<n_{j}$, we cannot have $n_{j}<n_{i}$, i.e., there is no $<$-cycle between the nodes.

(a) If $\neg\left(n_{j} \Longrightarrow^{*} n_{i}\right)$, we cannot have $n_{j} \prec n_{i}$ (and there is no cycle between the two nodes),

(b) If there is a simple cycle between the two nodes, then either the nodes are $<$-incomparable (i.e., we have a contradiction) or $n_{i}<n_{j} \wedge \neg\left(n_{j}<n_{i}\right)$, cf. Lemma D.4

(c) If there is (only) a non-simple cycle such that $n_{i} \Longrightarrow{ }^{*} n_{j}$, $n_{j} \Rightarrow^{*} n_{i}$, then, each path between $n_{i}$ and $n_{j}$ must go through $n_{3}$ such that $n_{3} \neq n_{j}$ and $n_{3} \neq n_{i}$, which contradicts $n^{\prime} \Longrightarrow n^{\prime \prime}$.

Given that there cannot be a $<$-cycle amongst the nodes in $N_{0}^{\prime \prime}$, the function must return at least one element (i.e., at least one top element satisfies the condition).

Lemma D.6. Let $S=\left(M_{\mathrm{p}}\right)_{\mathrm{p} \in \mathcal{P}}$ be a GMC system and $T S(S)=$ $\left(N, n_{0}, \hat{E}, \Longrightarrow\right)$. For all $n \in N$, if $n \stackrel{e_{1}}{\Longrightarrow} n_{1}$ and $n \stackrel{e_{2}}{\Longrightarrow} n_{2}$, then either

1. $n_{1} \stackrel{e_{2}}{\Rightarrow} n^{\prime}$ and $n_{2} \stackrel{e_{1}}{\Rightarrow} n^{\prime}$, for some $n^{\prime} \in N$, or

2. $\operatorname{snd}\left(e_{1}\right)=\operatorname{snd}\left(e_{2}\right)$ 
Proof. Direct from Lemma D.5 and Definition 3.5

Lemma D.7. Let $S=\left(M_{\mathrm{p}}\right)_{\mathrm{p} \in \mathcal{P}}$ be a GMC system and $T S(S)=$ $\left(N, n_{0}, \hat{E}, \Longrightarrow\right)$. For all $n \neq n^{\prime} \in N$, if $n \stackrel{e}{\Rightarrow} n_{1}, n^{\prime} \stackrel{e}{\Longrightarrow} n_{2}$, and $n \stackrel{e^{\prime}}{\Rightarrow} n^{\prime}$ then, $n_{1} \stackrel{e^{\prime}}{\Rightarrow} n_{2}$.

Proof. There are four cases depending on the machines involved in $e$ and $e^{\prime}$, and whether the event fired from $n$ is the same as the event fired from $n^{\prime}$ in $\hat{\delta}$.

1. If $\operatorname{id}(e) \cap \operatorname{id}\left(e^{\prime}\right)=\varnothing$, i.e., the events are independent, the result follows by definition of $T S(S)$ : we have $n_{1} \stackrel{e^{\prime}}{\Rightarrow} n_{2}$.

2. If $\operatorname{id}(e)=\operatorname{id}\left(e^{\prime}\right)$ and $\left(n, e, n_{1}\right),\left(n^{\prime}, e, n_{2}\right) \in \hat{\delta}$, then we have $\forall \mathrm{p} \in \operatorname{id}(e): n[\mathrm{p}]=n^{\prime}[\mathrm{p}]$, which contradicts the fact that $n \neq$ $n^{\prime}$.

3. If $\operatorname{id}(e) \cap \operatorname{id}\left(e^{\prime}\right) \neq \varnothing$ and $\left(n, e, n_{1}\right) \notin \hat{\delta}$ or $\left(n^{\prime}, e, n_{2}\right) \notin \hat{\delta}$, then we must have, without loss of generality, $\left(n, e, n_{1}\right),\left(n^{\prime}, e^{\prime \prime}, n_{2}\right) \in$ $\hat{\delta}$, such that $e \bowtie e^{\prime \prime}$ and $e \neq e^{\prime \prime}$. By definition of $\bowtie$, we must have $e=\left(q_{\mathrm{s}}, q_{\mathrm{r}}, \mathrm{s} \rightarrow \mathrm{r}: a\right)$ and $e^{\prime \prime}=\left(q_{\mathrm{s}}^{\prime \prime}, q_{\mathrm{r}}^{\prime \prime}, \mathrm{s} \rightarrow \mathrm{r}: a\right)$, with $q_{\mathrm{s}} \neq q_{\mathrm{s}}^{\prime}$ or $q_{\mathrm{r}} \neq q_{\mathrm{r}}^{\prime}$. For each $\mathrm{p} \in \operatorname{id}(e) \cap \operatorname{id}\left(e^{\prime}\right)$, we have $\left(n[\mathrm{p}], e^{\prime} l_{\mathrm{p}}\right.$ ,$\left.n^{\prime}[\mathrm{p}]\right) \in \delta_{\mathrm{p}}$ by assumption and, by definition of $\bullet$, we must also have $\left(n_{1}[\mathrm{p}],\left.e^{\prime}\right|_{\mathrm{p}}, n_{2}[\mathrm{p}]\right) \in \delta_{\mathrm{p}}$. Thus, if $\operatorname{id}(e)=\operatorname{id}\left(e^{\prime}\right)$, we have the result immediately. If $\operatorname{id}(e) \neq \operatorname{id}\left(e^{\prime}\right)$, then the machine not involved in $e$ is still able to interact with p so to fire $e^{\prime}$, and we have the result.

4. If $\operatorname{id}(e) \cap \operatorname{id}\left(e^{\prime}\right) \neq \varnothing, \operatorname{id}(e) \neq \operatorname{id}\left(e^{\prime}\right)$, and $\left(n, e, n_{1}\right),\left(n^{\prime}, e, n_{2}\right) \in$ $\hat{\delta}$, then, it must be the case that one of the machine has a selfloop, so that we have three machines of the form:
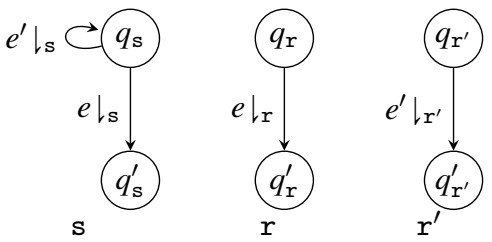

If there is $\left(q_{\mathrm{s}}^{\prime}, e^{\prime} l_{\mathrm{s}}, q_{\mathrm{s}}^{\prime}\right) \in \delta_{\mathrm{s}}$ then we have the result immediately (as above), otherwise there are two cases, depending on whether $\left(n_{1}, n^{\prime}\right) \in \ln \left(n, e, e^{\prime}\right)$.

(a) If $\left(n_{1}, n^{\prime}\right) \in \ln \left(n, e, e^{\prime}\right)$, then either the two branches commute, and we have the result; or case (2) of Definition 3.5. must hold. However, in both branches, machine $r$ is able to execute $e l_{r}$ as a first action (since $r \notin \operatorname{id}\left(e^{\prime}\right)$ by assumption). This means that the two branches must merge, i.e., once in state $q_{\mathrm{s}}^{\prime}$, s must be able to interact with $\mathrm{r}^{\prime}$ such that $r^{\prime}$ reaches $q_{\mathrm{r}^{\prime}}^{\prime}$. In addition, by representability, the selfloop at s must appear in $T S(S)$, which means there must be two branches in $T S(S)$, one that leads to a configuration where machines $\mathrm{s}, \mathrm{r}$, and $\mathrm{r}^{\prime}$ are in states $q_{\mathrm{s}}, q_{\mathrm{r}}$, and $q_{\mathrm{r}^{\prime}}$, respectively (where the self-loop is not represented); and one branch that leads to a configuration where the self-loop appears. This contradicts case (2) of Definition 3.5 since $r$ would have the same first actions in both branches, while its behaviour must be different in both branches (i.e., the self-loop appears in one but not in the other). (b) If $\left(n_{1}, n^{\prime}\right) \notin \ln \left(n, e, e^{\prime}\right)$, we must have the following situation (by definition of the last node function):

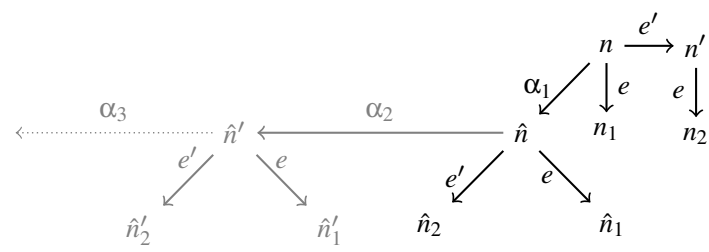

Indeed, since $\left(n_{1}, n^{\prime}\right) \notin \ln \left(n, e, e^{\prime}\right)$, there must be a successor of $n$ that fires both $e$ and $e^{\prime}$, i.e, $\hat{n}$ in the diagram above. Note that by, Lemma D.2 $\hat{n}$ cannot be a target of $e$ or $e^{\prime}$.

i. If $\left(\hat{n}_{1}, \hat{n}_{2}\right) \in \ln \left(\hat{n}, e, e^{\prime}\right)$. We show that $e$ must also be fireable from $\hat{n}_{2}$, by contradiction. Since $e^{\prime} l_{\mathrm{s}}$ is a selfloop in $\mathbf{s}$ (and the machines are deterministic), $\mathbf{s}$ is still able to fire $e l_{\mathrm{s}}$ after $e^{\prime} l_{\mathrm{s}}$; and since $\mathrm{r} \notin \operatorname{id}\left(e^{\prime}\right)$, the only way $e$ would not be fireable from $\hat{n}_{2}$ is if $r \in \mathrm{id}\left(\alpha_{1}\right)$ and $\left.\forall q \in Q_{\mathrm{r}}:\left(\hat{n}[\mathrm{r}], e l_{\mathrm{r}}, q\right) \notin \delta_{\mathrm{r}}\right)$, which contradicts the fact that $\hat{n} \stackrel{e}{\Longrightarrow}$. We can now repeat the argument with $\alpha_{1}=e^{\prime}, \hat{n}=n^{\prime}$, and $\hat{n}_{1}=n_{2}$.

ii. If $\left(\hat{n}_{1}, \hat{n}_{2}\right) \notin \ln \left(\hat{n}, e, e^{\prime}\right)$, then we can repeat the argument (cf. faded part of the picture) until we reach a pair of nodes that is in $\ln \left({ }_{-}, e, e^{\prime}\right)$. We know that such a pair exists by LemmaD.5

Definition D.1. Let $S=\left(M_{\mathrm{p}}\right)_{\mathrm{p} \in \mathcal{P}}, T S(S)=\left(N, n_{0}, \hat{E}, \Longrightarrow\right), \mathrm{p} \in \mathcal{P}$, $q \in Q_{\mathrm{p}}$, and $\left(q, \ell_{1}, q_{1}\right),\left(q, \ell_{2}, q_{2}\right) \in \delta_{\mathrm{p}}$, with $\ell_{1} \neq \ell_{2}$, we write

$$
\left(q, \ell_{1}, q_{1}\right) \smile\left(q, \ell_{2}, q_{2}\right)
$$

iff there exists $n \in N$ such that:

$$
\begin{aligned}
& \text { 1. } n[\mathrm{p}]=q \\
& \text { 2. } n \stackrel{e_{1}}{\Rightarrow} n_{1}, n \stackrel{e_{1}}{\Rightarrow} n_{2}, \\
& \text { 3. }\left(n_{1}, n_{2}\right) \in \ln \left(n, e_{1}, e_{2}\right) \\
& \text { 4. }\left\{\ell_{i}\right\}=h d\left(\left\{e_{i} l_{\mathrm{p}} \cdot \varphi \mid \varphi \in L T\left(S, n_{i}, \mathrm{p}\right)\right\}\right) \text {, for } i \in\{1,2\} .
\end{aligned}
$$

We write $\left(q, \ell_{1}, q_{1}\right) \asymp\left(q, \ell_{2}, q_{2}\right)$ iff either $(i)\left(q, \ell_{1}, q_{1}\right) \smile\left(q, \ell_{2}, q_{2}\right)$; or (ii) there is $\left(q, \ell^{\prime}, q^{\prime}\right) \in \delta_{\mathrm{p}}$ such that $\left(q, \ell_{1}, q_{1}\right) \smile\left(q, \ell^{\prime}, q^{\prime}\right)$ and $\left(q, \ell^{\prime}, q^{\prime}\right) \asymp\left(q, \ell_{2}, q_{2}\right)$.

Lemma D.8 (Verified branches). Let $S=\left(M_{\mathrm{p}}\right)_{\mathrm{p} \in \mathcal{P}}$ be a $G M C$ system and $\operatorname{TS}(S)=\left(N, n_{0}, \hat{E}, \Longrightarrow\right)$. For all $\mathrm{p} \in \mathcal{P}$ and for all $q \in$ $Q_{\mathrm{p}}$, if $\left(q, \ell_{1}, q_{1}\right),\left(q, \ell_{2}, q_{2}\right) \in \delta_{\mathrm{p}}$ (with $\left.\ell_{1} \neq \ell_{2}\right)$, then $\left(q, \ell_{1}, q_{1}\right)=$ $\left(q, \ell_{2}, q_{2}\right)$.

Proof. First, we show the following:

$$
\forall\left(q, \ell_{1}, q_{1}\right) \in \delta_{\mathrm{p}}, \exists\left(q, \ell_{2}, q_{2}\right) \in \delta_{\mathrm{p}}:\left(q, \ell_{1}, q_{1}\right) \smile\left(q, \ell_{2}, q_{2}\right)
$$

By Lemma D.1. for each $\left(q, \ell_{2}, q_{2}\right) \in \delta_{\mathrm{p}}$ there is $n$ such that $n[\mathrm{p}]=$ $q$,

$$
\begin{gathered}
n \stackrel{\pi_{1}}{\Rightarrow} n_{1} \stackrel{e_{1}}{\Rightarrow} \text { with } \pi_{1} l_{\mathrm{p}}=\varepsilon \wedge e_{1} l_{\mathrm{p}}=\ell_{1} \\
\quad \text { and } \\
n \stackrel{\pi_{2}}{\Rightarrow} n_{2} \stackrel{e_{2}}{\Longrightarrow} \text { with } \pi_{2} l_{\mathrm{p}}=\varepsilon \wedge e_{2} l_{\mathrm{p}}=\ell_{2}
\end{gathered}
$$

Choose $n, \ell_{2}, \pi_{1}$, and $\pi_{2}$ such that $\pi_{1}$ is the smallest, i.e., there is no other node $n^{\prime}$ such (5) holds and $\pi_{1}$ is strictly smaller, for some $\ell_{2}$. In other words, $n$ is the last node from which p can "choose" to fire either $\ell_{1}$ or another action $\left(\ell_{2}\right)$. Let $k=|h d(L T(S, n, \mathrm{p}))|$ and recall that $\ell_{1}, \ell_{2} \in h d(L T(S, n, \mathrm{p}))$ by assumption.

We first show the result for $k=2$.

1. If $n$ is such that $n \stackrel{e_{1}}{\Longrightarrow}$ and $n \stackrel{e_{2}}{\Longrightarrow}$, with $e_{i} l_{\mathrm{p}}=\ell_{i}(i \in\{1,2\})$, then, by Lemma D.5 there is $\hat{n}, \hat{n}_{1}, \hat{n}_{2} \in N$ such that $\hat{n} \stackrel{e_{1}}{\Longrightarrow} \hat{n}_{1}$ and $\hat{n} \stackrel{e_{2}}{\Rightarrow} \hat{n}_{2}$, with $\left(\hat{n}_{1}, \hat{n}_{2}\right) \in \ln \left(\hat{n}, e_{1}, e_{2}\right)$. Thus, we have $\left(q, \ell_{1}, q_{1}\right) \smile$ $\left(q, \ell_{2}, q_{2}\right)$. 
2. If $n$ is such that $n \stackrel{e_{1}}{\Rightarrow}, n \stackrel{e_{2}}{\Rightarrow}$, with $e_{i} \iota_{\mathrm{p}} \neq \ell_{i}(i \in\{1,2\})$, and $\left(n_{1}, n_{2}\right) \in \ln \left(n, e_{1}, e_{2}\right)$, then we have $\left(q, \ell_{1}, q_{1}\right) \smile\left(q, \ell_{2}, q_{2}\right)$, since $n$ is the latest node and $k=2$.

3. If $n$ is such that $n \stackrel{e_{1}}{\Rightarrow}, n \stackrel{e_{2}}{\Rightarrow}$, with $e_{i} l_{\mathrm{p}} \neq \ell_{i}(i \in\{1,2\})$, and $\left(n_{1}, n_{2}\right) \notin \ln \left(n, e_{1}, e_{2}\right)$, we must have the following situation, where $e_{i}^{\prime} l_{\mathrm{p}}=\ell_{i}$,

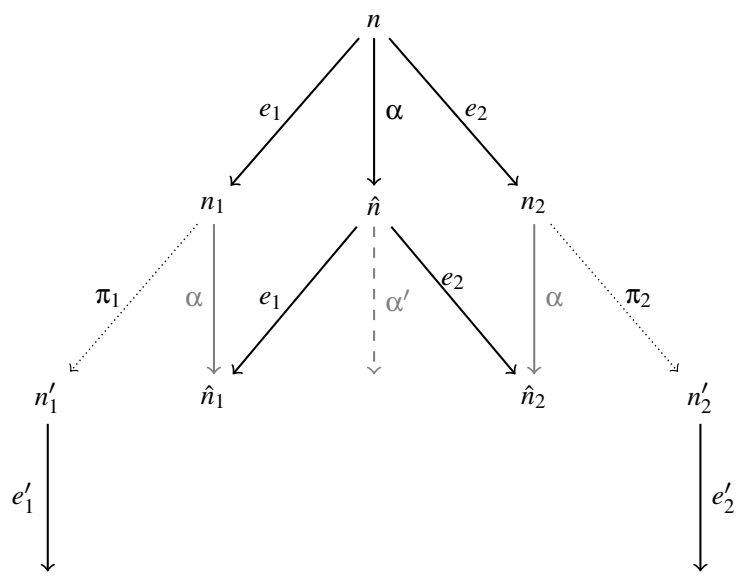

Since, $\left(n_{1}, n_{2}\right) \notin \ln \left(n, e_{1}, e_{2}\right)$, it must be the case that there is a successor of $n$ that is able to fire both $e_{1}$ and $e_{2}$, i.e., $\hat{n}$ here. Note that by, Lemma D.2 $\hat{n}$ cannot be a target of $e_{1}$ or $e_{2}$. In addition, by LemmaD.7 there must be an event $\alpha$ between both $n_{i}$ and $\hat{n}_{i}(i \in\{1,2\})$, cf. faded $\alpha$-labelled edges in the diagram above.

By assumption $(k=2)$, we have $\left\{\ell_{i}\right\}=h d\left(L T\left(S, n_{i}, \mathrm{p}\right)\right)$ (otherwise, $n$ would not be the latest node); thus $\ell_{2} \neq \alpha l_{p} \neq \ell_{1}$.

For $i \in\{1,2\}$, we reason as follows:

- If case (1) of Definition 3.5 applies to the branching at $n_{i}$, then $e_{i}^{\prime}$ can also be fired from $\hat{n}_{i}$, and we have the result.

- If case 22 of Definition 3.5 applies to the branching at $n_{i}$, then either (i) machine p must execute an action different from $\ell_{i}$ in the branch corresponding to $\pi_{i}$. However, this contradicts the fact that $\ell_{2}$ cannot appear in $\pi_{1}^{\prime} l_{\mathrm{p}}$. Or, (ii) there is a merging node $n^{\prime \prime}$, reachable from both $n_{1}$ and $n_{2}$, and two paths (from $n^{\prime \prime}$ to $n_{i}$ ) such that machine $\mathrm{p}$ has an empty behaviour on both; which contradicts our assumption that $n$ is the last node such that $\ell_{1}$ and $\ell_{2}$ are fireable.

Since $\ell_{1}$ and $\ell_{2}$ are the only actions fireable from $q$, we conclude that each action is executed from $\hat{n}_{1}$ and $\hat{n}_{2}$, respectively, and we obtain the result.

4. If $\left(\hat{n}_{1}, \hat{n}_{2}\right) \notin \ln \left(\hat{n}, e_{1}, e_{2}\right)$, then we repeat the same argument until we find a pair that is included in $\ln \left(-, e_{1}, e_{2}\right)$, and we know there is such a pair by Lemma D.5. cf. faded $\alpha^{\prime}$ edge in the diagram above (or leads to a contradiction).

If $k>2$, then we reason similarly, with $k$ events $e_{i}$ and nodes $n_{i}$, where each path cannot execute any actions from $\mathrm{p}$ without leading to a contradiction. We then conclude that case (1) of Definition 3.5 must apply for each path and we finally reach a pair of nodes in $\ln \left(\hat{n}, e_{1}, e_{2}\right)$, as above.

Second, having established (4), we show that

$$
\forall\left(q, \ell_{1}, q_{1}\right) \neq\left(q, \ell_{2}, q_{2}\right) \in \delta_{\mathrm{p}}:\left(q, \ell_{1}, q_{1}\right)=\left(q, \ell_{2}, q_{2}\right)
$$

We show this by contradiction, if (6) does not hold, we must have partitions of outgoing transitions from $q$ such that the result hold within each partition, but not across two partitions, i.e., we have a situation of the form below, where $n$ is the last node such that all transitions of $\mathrm{p}$ from $q$ are taken.

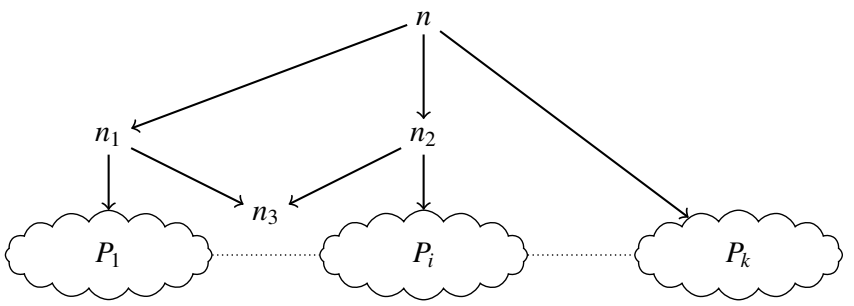

However, between two partitions either $(i)$ case (2) of Definition 3.5 applies, i.e., the branches do not commute nor merge (cf. right-hand side of the diagram above) and thus, there must be a node $n$ and two events $e_{1}$ and $e_{2}$ which will be checked for, by Lemma D.5. or (ii) case (1) of Definition 3.5 applies (cf. left-hand side of the diagram), in which case there is two other nodes $n_{1}$ and $n_{2}$, which commute (or merge, cf., item (ii) in 2a) of Definition 3.5 to a third node, $n_{3}$, and we can inductively repeat the argument in $(i)$ where $n_{1}$ and $n_{2}$ replace $n$. Note that it must be the case that p executes some actions from nodes $n_{3}$, thus linking two partitions together.

\section{D.4 Proof of Theorem 3.1 (Soundness)}

Theorem 3.1 (Soundness). If $S$ is GMC, then it is safe (no orphan message, deadlock, and unspecified reception configurations).

Proof. Absence of orphan message configuration and unspecified reception configurations follow directly from Lemma D.12

We show absence of deadlock configuration, by contradiction, using Lemma D.13 Assume there is a deadlock configuration $s \in$ $R S(S)$ such that $s=(\vec{q} ; \vec{\varepsilon}), n=\vec{q}$, and $\left(q_{\mathrm{r}}, \mathrm{sr} ? a, q_{\mathrm{r}}^{\prime}\right) \in \delta_{\mathrm{r}}$ for some $r \in \mathcal{P}$. By Lemma D.13, we have $n_{0} \Longrightarrow^{*} n$, and since $S$ is representable, there must be an execution in which sr? $a$ is taken. Moreover, by Lemma D.12, there is no unspecified reception configuration, so it must be the case that there is a branching in $M_{\mathrm{r}}$ such that the machine is expecting a message $a$ in one branch, but not in the other (reasoning in a similar fashion to the proof of Lemma D.12). The branching in $M_{\mathrm{r}}$ must be reflected in $T S(S)$ such that there is a branch going to $n$ and another branch where the interaction on $a$ takes place. However, in the $n$ branch, the behaviour of $r$ is empty (deadlock) and thus we obtain a contradiction with Definition 3.5 since $r$ executes $s r ? a$ in one branch and does nothing (i.e., $\varepsilon$ ) in the other branch.

Lemma D.9. Let $S=\left(M_{\mathrm{p}}\right)_{\mathrm{p} \in \mathcal{P}}$ be a GMC system and $T S(S)=$ $\left(N, n_{0}, \hat{E}, \Longrightarrow\right)$. For all $\mathrm{p} \in \mathcal{P}$ and for all $q \in Q_{\mathrm{p}}$, if $\left(q, \ell_{1}, q_{1}\right)$, $\left(q, \ell_{2}, q_{2}\right) \in \delta_{\mathrm{p}}$ (with $\left.\ell_{1} \neq \ell_{2}\right)$, and there is $n \in N, n[\mathrm{p}]=q$, such that $n \stackrel{e_{i}}{\Rightarrow}$ with $e_{i} l_{\mathrm{p}}=\ell_{i}$, then $\left(q, \ell_{1}, q_{1}\right) \smile\left(q, \ell_{2}, q_{2}\right)$.

Proof. Direct from Definition D.1 and Lemma D.5

Lemma D.10. Let $S=\left(M_{\mathrm{p}}\right)_{\mathrm{p} \in \mathcal{P}}$ be a GMC system and $T S(S)=$ $\left(N, n_{0}, \hat{E}, \Longrightarrow\right)$. For all $\mathrm{p} \in \mathcal{P}$ and for all $q \in Q_{\mathrm{p}}$, if $\left(q, \ell_{1}, q_{1}\right)$, $\left(q, \ell_{2}, q_{2}\right) \in \delta_{\mathrm{p}}$ (with $\left.\ell_{1} \neq \ell_{2}\right)$, such that $\ell_{1}$ (resp. $\left.\ell_{2}\right)$ is a send action (resp. receive) action and $\left(q, \ell_{1}, q_{1}\right) \smile\left(q, \ell_{2}, q_{2}\right)$, then there is $n \in N, n[\mathrm{p}]=q$, such that $n \stackrel{e_{i}}{\Rightarrow}$ with $e_{i} l_{\mathrm{p}}=\ell_{i}$.

Proof. By contradiction, assume there is no such $n \in N$. Then for all $n \stackrel{\pi_{i}}{\Longrightarrow} n_{i} \stackrel{e_{i}}{\Longrightarrow}$, with $\pi_{i} l_{\mathrm{p}}=\varepsilon$ and $e_{i} l_{\mathrm{p}}=\ell_{i},\left|\pi_{i}\right|>0$. Observe that such nodes exists since $\left(q, \ell_{1}, q_{1}\right) \smile\left(q, \ell_{2}, q_{2}\right)$. This implies that case 11 of Definition 3.5 cannot apply, and case 2) of Definition 3.5 cannot apply either (since there is no unique sender); which contradicts the fact that $S$ is GMC. 
Lemma D.11 (Mixed choice). Let $S=\left(M_{\mathrm{p}}\right)_{\mathrm{p} \in \mathcal{P}}$ be a GMC system, for all $\mathrm{p} \in \mathcal{P}$ and for all $q \in Q_{\mathrm{p}}$ such that $\left(q, \ell, q^{\prime}\right),\left(q, \ell^{\prime}, q^{\prime \prime}\right) \in \delta_{\mathrm{p}}$ for some $q^{\prime}, q^{\prime \prime} \in Q_{\mathrm{p}}$, if $\ell$ is a send action and $\ell^{\prime}$ is a receive action, then there is $\hat{q} \in Q_{\mathrm{p}}$ such that $\left(q^{\prime}, \ell^{\prime}, \hat{q}\right) \in \delta_{\mathrm{p}}$ and $\left(q^{\prime \prime}, \ell, \hat{q}\right) \in \delta_{\mathrm{p}}$.

Proof. By Lemma D.8 we must have $\left(q, \ell, q^{\prime}\right) \asymp\left(q, \ell^{\prime}, q^{\prime \prime}\right)$.

If $\left(q, \ell, q^{\prime}\right) \smile\left(q, \ell^{\prime}, q^{\prime \prime}\right)$, the result follows directly by Lemma D.10 If $\neg\left(\left(q, \ell, q^{\prime}\right) \smile\left(q, \ell^{\prime}, q^{\prime \prime}\right)\right)$, then there must be a chain of transitions $\left(q, \ell_{i}, q_{i}\right)$ such that

$$
\left(q, \ell_{1}, q_{1}\right) \smile \ldots \smile\left(q, \ell_{k}, q_{k}\right) \quad k>2
$$

where $\ell_{1}=\ell, \ell_{k}=\ell^{\prime}, q_{1}=q^{\prime}$, and $q_{k}=q^{\prime \prime}$. We show that for all $1 \leqslant i \neq j \leqslant k: \ell_{i}$ is a send action and $\ell_{j}$ is a receive action, then there is $\hat{q} \in Q_{\mathrm{p}}$ such that $\left(q_{i}, \ell_{j}, \hat{q}\right) \in \delta_{\mathrm{p}}$ and $\left(q_{j}, \ell_{i}, \hat{q}\right) \in \delta_{\mathrm{p}}$. We show the result by induction on the length $k$ of the smallest chain of transitions for which (7) holds. Assume $k=3$, then there are two cases

- If $\ell_{2}$ is a send action, then it must commute with $\ell_{3}$, by Lemma D.10 and Definition 3.5 By LemmaD.9, we must also have $\left(q, \ell_{1}, q_{1}\right) \smile\left(q, \ell_{3}, q_{3}\right)$, and we are done.

- If $\ell_{2}$ is a receive action, then it must commute with $\ell_{1}$, by Lemma D.10 and we reason as above.

The inductive case follows straightforwardly. Assume the result holds for $k>3$ and let us show it holds for $k+1$. If $\ell_{k}$ is a send action, it must commute with $\ell_{k+1}$, which implies that there is a smaller chain $=$-linking $\ell_{k+1}$ with $\ell_{1}$ (as above). If $\ell_{k}$ is a receive action, then it must commute with all the send actions $\ell_{i}$ such that $i<k$, thus there is a smaller chain $\asymp$-linking $\ell_{k+1}$ with a send action, and we are done by induction hypothesis.

Lemma D.12. Let $S=\left(M_{\mathrm{p}}\right)_{\mathrm{p} \in \mathcal{P}}$ be a GMC system for all $s \in R S(S)$, if $s \stackrel{\mathrm{sr} ! a}{\longrightarrow} s_{1} \stackrel{\varphi}{\rightarrow}_{s_{2}}$, with $\mathrm{sr} ? a \notin \varphi$, then $s_{2} \rightarrow^{*} s_{3} \stackrel{\mathrm{sr} ? a}{\longrightarrow} s_{4}$.

Proof. By contradiction, assume that there is $s \in R S(S)$ such that $s \stackrel{\text { sr! } a}{\longrightarrow} s^{\prime}$ and for all $\varphi$ such that $s^{\prime} \stackrel{\varphi}{\rightarrow}$, we have sr? $a \notin \varphi$. In addition, assume that $s$ is the first configuration (from $s_{0}$ ) such that a sent message cannot be received.

Assume that the state of $\mathrm{s}$ in $s$ is $q_{\mathrm{s}}$. Since $S$ is representable, there must be $s^{\prime \prime} \in R S(S)$, where the state of $\mathrm{s}$ in $s^{\prime \prime}$ is $q_{\mathrm{s}}$, such that $s^{\prime \prime} \stackrel{\text { sr! } a}{\longrightarrow} \stackrel{\text { sr? } a}{\longrightarrow}$, i.e., there is an execution where the message $a$ sent by $s$ is received by $r$. If the message cannot be received by $r$ from $s$, it means that either

1. $r$ is unable to fire the action sr? $a$ because it is expecting another message, i.e., it is blocked by a transition pr? $b$, or

2. there is a branching in $M_{\mathrm{r}}$ such that in one of the branch $a$ is received but not in the other.

Case (1) implies that there is another configuration before $s$ where a message is not received, thus we only consider case 2 .

Let us consider the last state $q \in Q_{\mathrm{r}}$ such that $\left(q, \ell_{1}, q_{1}\right),\left(q, \ell_{2}, q_{2}\right) \in$ $\delta_{\mathrm{r}}\left(\ell_{1} \neq \ell_{2}\right)$ and there is no sr?a transitions fireable from $q_{1}$ (before any other transition receiving from $s$ ) and the first transition receiving from $\mathrm{s}$ after $q_{2}$ is sr? $a$. Observe that since $q$ is the last state where such a branching occur, there cannot be $q^{\prime} \in Q_{\mathrm{r}}$ such that $\left(q_{1}, \ell_{2}, q^{\prime}\right),\left(q_{2}, \ell_{1}, q^{\prime}\right) \in \delta_{\mathrm{r}}$.
Essentially, we have the following situation:

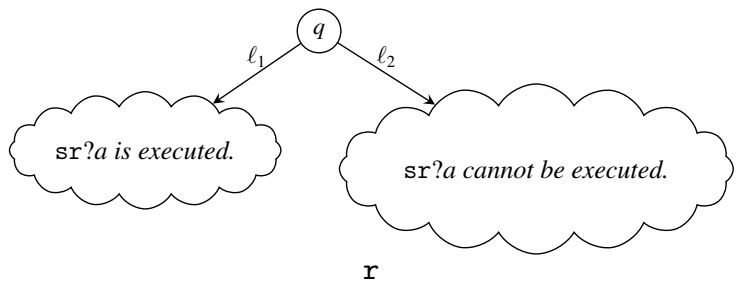

where $\mathrm{r}$ cannot execute $\mathrm{sr}$ ? a once it has taken the transition $\ell_{2}$. We have the following cases:

1. $\ell_{1}$ is a send action and $\ell_{2}$ is a receive action,

2. both $\ell_{1}$ and $\ell_{2}$ are send actions, or

3 . both $\ell_{1}$ and $\ell_{2}$ are receive actions.

Following Lemma D.11, case (1) cannot happen since it would imply that the branches commute.

Observe that if there are more than two transitions outgoing $q$, we can partition them into two sets: $(i)$ the transitions after which $a$ is a received and (ii) the transitions after which $a$ is not received. By Lemma D.8, there must be, at least, one transition $\left(q, \ell_{2}, q_{2}\right)$ in $(i)$ and one transition $\left(q, \ell_{1}, q_{1}\right)$ in (ii) such that $\left(q, \ell_{1}, q_{1}\right) \smile$ $\left(q, \ell_{2}, q_{2}\right)$. Hereafter, we only consider these two transitions. Case (2) of Definition 3.5 must apply to a branching node $n$ such that $n[\mathrm{p}]=q$ and the first action of $\mathrm{r}$ in one branch is $\ell_{1}$ and the first action of $r$ in the other branch is $\ell_{2}$, by definition of $\smile$. Note that case (ii) of 2a cannot apply for $r$ since it would otherwise mean that there is a later branching in machine $r$ such that it can "choose" whether to receive $a$ or not; i.e., $r$ must be involved in the choice.

Case (2). Then, $r$ is the selector at node $n$. Following Definition 3.5, there are two sub-cases: Machine s executes a receive action before sending another action. This implies that there must be a branching in $\mathrm{s}$ before the transition $\mathrm{sr} ! a$, which means that $\mathrm{sr} ! a$ can only be executed once the $\ell_{2}$-branch has been executed. Thus, $r$ cannot make a "bad choice": the two branches are mutually exclusive in all possible executions.

Indeed, if $r$ was able to make another choice, it would mean that it could choose between receiving $\ell_{1}$ and $\ell_{2}$, i.e., there is no $\triangleleft$-dependency between $\ell_{1}$ and $\ell_{2}$. More formally, assume we have $\left(q, \mathrm{~s}_{1} \mathrm{r} ? \ell_{1}, q_{1}\right),\left(q, \mathrm{~s}_{2} \mathrm{r} ? \ell_{2}, q_{2}\right) \in \delta_{\mathrm{r}}$. By representability, there must be a node $n \in N$ such that, for $i \in\{1,2\}, n \stackrel{\pi_{i}}{\Longrightarrow} n_{i} \stackrel{e_{i}}{\Rightarrow}$ with $e_{i} l_{r}=\mathrm{s}_{\mathrm{i}} \mathrm{r} ? \ell_{i}, \pi_{i} l_{\mathrm{r}}=\varepsilon$, and $n[\mathrm{r}]=q$. Also, by GMC and the fact that we assumed that the non-reception at $r$ is the first one in the system, $r$ must be able to receive both messages from $s_{1}$ and $s_{2}$ in each branch, i.e., we must have, for $i \neq j \in\{1,2\}, n_{i} \stackrel{e_{i}}{\Longrightarrow} \Longrightarrow$ with $e_{j} l_{r}=\mathrm{s}_{j} r ? \ell_{j}$. If $\mathrm{s}_{1}=\mathrm{s}_{2}$, the two actions are always mutually exclusive (since the channels are message order preserving) and thus there is always a trivial $\triangleleft$-dependency. If $s_{1} \neq s_{2}$, we must have a $\triangleleft$-chain between the two corresponding interactions in both branches. The shortest of such chains is as follows, with $i \neq j \in$ $\{1,2\}$,

$$
\mathrm{s}_{\mathrm{i}} \rightarrow \mathrm{r}: \ell_{i} \triangleleft \mathrm{r} \rightarrow \mathrm{s}_{\mathrm{j}}: a \triangleleft \mathrm{s}_{\mathrm{j}} \rightarrow \mathrm{r}: \ell_{j}
$$

which implies that $r$ cannot choose between receiving either message in any asynchronous execution.

Case (3). This means that another machine selects the branch:

- If $s$ is the selector, then there must be a branching in machine $s$ such that s sends a different message to all participants involved in the choice (possibly through other participants), including $r$. This implies that $r$ must be aware of which branch $s$ has chosen and thus cannot make a "bad choice" either.

- If $\mathrm{s}$ is not the selector, there must be another participant making the decision and we reason as in case (2) above. 


\section{D.5 Proof of Reachability}

The following lemma states the reachability property, which is important to apply Cortadella's algorithm.

Lemma 4.1. If $S$ is $G M C$ and $T S(S)=\left(N, n_{0}, \hat{E}, \Longrightarrow\right)$, then $\forall n \in$ $N: n_{0} \Longrightarrow^{*} n$.

Proof. By contradiction, assume there is $(\vec{q}, \vec{\varepsilon}) \in R S_{1}(S)$ such that $n_{0} \Longrightarrow{ }^{*} \vec{q}$ and $\left(\vec{q}^{\prime}, \vec{\varepsilon}\right) \in R S_{1}(S)$ such that $\left(\vec{q}^{\prime}, \vec{\varepsilon}\right)$ is reachable by $1-$ bounded execution from $(\vec{q}, \vec{\varepsilon})$, but not by a synchronous execution. This implies that there is a dependency chain between the two configurations. The smallest such chain is:

$$
(\vec{q}, \vec{\varepsilon}) \stackrel{\mathrm{sr} ! a}{\longrightarrow} \stackrel{\mathrm{rs} ! b}{\longrightarrow} \stackrel{\mathrm{rs} ? b}{\longrightarrow} \stackrel{\mathrm{sr} ? a}{\longrightarrow}\left(\vec{q}^{\prime}, \vec{\varepsilon}\right)
$$

i.e., it is not possible to swap actions $r s ! b$ and $s r ? a$, thus there is no synchronous counterpart for this execution. Such an execution implies that we have machines of the form:

$$
\mathrm{s}: q_{1} \stackrel{\mathrm{sr} ! a}{\longrightarrow} q_{2} \stackrel{\mathrm{rs} ? b}{\longrightarrow} q_{3} \quad \mathrm{r}: q_{1}^{\prime} \stackrel{\mathrm{rs} ! b}{\longrightarrow} q_{2}^{\prime} \stackrel{\mathrm{sr} ? a}{\longrightarrow} q_{3}^{\prime}
$$

By representability, each execution must be represented in $T S(S)$, thus, there must be two branches in each machine, so that both branches appear in $T S(S)$ :

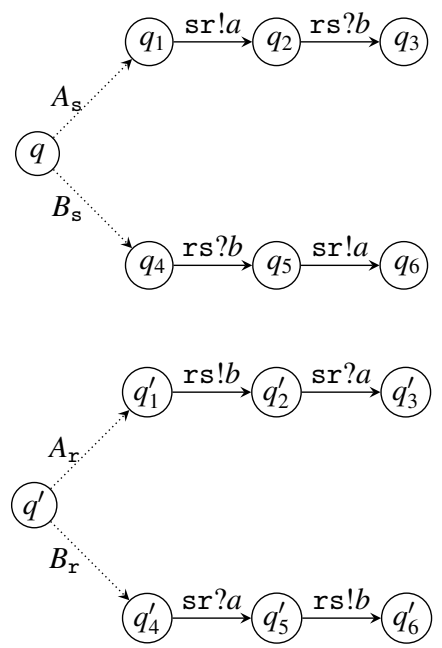

By assumption, branches $A_{\mathrm{s}}$ and $A_{\mathrm{r}}$ can be executed synchronously, since $n_{0} \Longrightarrow * \vec{q}$, while, by representability, $A_{\mathrm{S}}$ and $B_{\mathrm{r}}$ (resp. $B_{\mathrm{S}}$ and $A_{\mathrm{r}}$ ) must be executable synchronously.

- If $q_{1}=q_{4}$ or $q_{1}^{\prime}=q_{4}^{\prime}$, we obtain a contradiction with the assumption that $\left(\vec{q}^{\prime}, \vec{\varepsilon}\right)$ is not reachable by a synchronous execution.

- Hence, if $q_{1} \neq q_{4}$ and $q_{1}^{\prime} \neq q_{4}^{\prime}$, there are three branches in $T S(S):\left(A_{\mathrm{s}}, A_{\mathrm{r}}\right),\left(A_{\mathrm{s}}, B_{\mathrm{r}}\right)$, and $\left(B_{\mathrm{s}}, A_{\mathrm{r}}\right)$. This implies that machine $\mathrm{s}$ (resp. $\mathrm{r}$ ) has the same first actions in branches $\left(A_{\mathrm{s}}, A_{\mathrm{r}}\right)$ and $\left(A_{\mathrm{S}}, B_{\mathrm{r}}\right)$ (resp. $\left(A_{\mathrm{S}}, A_{\mathrm{r}}\right)$ and $\left.\left(B_{\mathrm{S}}, A_{\mathrm{r}}\right)\right)$. Thus, either the branches commute, i.e., $q_{1}=q_{4}, q_{3}=q_{6}, q_{1}^{\prime}=q_{4}^{\prime}, q_{3}^{\prime}=q_{6}^{\prime}$, or there is a path in s (resp r) such that both branches merge, either way, this contradicts the fact that $\left(\vec{q}^{\prime}, \vec{\varepsilon}\right)$ is not reachable by a synchronous execution. If the branches do not commute nor merge, then the system is not GMC, which contradicts our assumptions.

Lemma D.13. If $S$ is $G M C$ and $T S(S)=\left(N, n_{0}, \hat{E}, \Longrightarrow\right)$, then $\forall(\vec{q}, \vec{\varepsilon}) \in R S(S): n_{0} \Longrightarrow^{*} \vec{q}$.
Proof. The proof is similar to the proof of Lemma 4.1. By contradiction, assume there is $s \in R S_{2}(S) \backslash R S_{1}(S)$ such that $s=(\vec{q}, \vec{\varepsilon})$ and $\neg\left(n_{0} \Rightarrow^{*} \vec{q}\right)$. Then, there should be an execution of the form

$$
s_{0} \stackrel{\varphi_{1}}{\longrightarrow} s_{1} \stackrel{\mathrm{sr} ! a_{a}}{\longrightarrow} s_{2} \stackrel{\varphi_{2}}{\longrightarrow} s_{3} \stackrel{\mathrm{sr} ! b}{\longrightarrow} s_{4} \stackrel{\varphi_{3}}{\longrightarrow} s_{5} \stackrel{\mathrm{sr} ? a}{\longrightarrow} s
$$

with $s_{1} \in R S_{1}(S)$, stable, $s_{3} \in R S_{1}(S)$ and $s_{4} \in R S_{2}(S) \backslash R S_{1}(S)$, such that there is a dependency chain in $\varphi_{3}$ between $s r ! b$ and sr? $a$ (otherwise $s$ would be reachable by a 1-bounded execution). The smallest such chain is of the form $r s ! c \cdot r s ? c$, thus let $\varphi_{2}=$ rs!c.rs?c. By representability, as in the proof of the lemma above, there must be two branches in each machine so that each trace can be executed synchronously, i.e.,

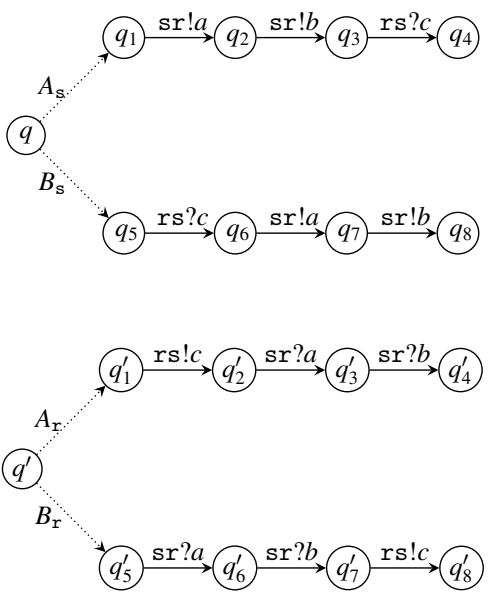

Branches $A_{\mathrm{s}}$ and $A_{\mathrm{r}}$ can be executed synchronously, since $s_{1}$ is stable and $s_{1} \in R S_{1}(S)$ (cf. Lemma 4.1) By representability, $A_{\mathrm{S}}$ and $B_{\mathrm{r}}$ (resp. $B_{\mathrm{S}}$ and $A_{\mathrm{r}}$ ) must be executable synchronously. Hence, if $q_{1} \neq q_{5}$ and $q_{1}^{\prime} \neq q_{5}^{\prime}$, there are three branches in $T S(S):\left(A_{\mathrm{S}}, A_{\mathrm{r}}\right)$, $\left(A_{\mathrm{s}}, B_{\mathrm{r}}\right)$, and $\left(B_{\mathrm{s}}, A_{\mathrm{r}}\right)$. This implies that machine $\mathrm{s}$ (resp. $\left.\mathrm{r}\right)$ has the same first actions in branches $\left(A_{\mathbf{S}}, A_{\mathrm{r}}\right)$ and $\left(A_{\mathbf{S}}, B_{\mathrm{r}}\right)$ (resp. $\left(A_{\mathbf{S}}, B_{\mathrm{r}}\right)$ and $\left.\left(B_{\mathrm{s}}, A_{\mathrm{r}}\right)\right)$. Thus, either the branches commute, i.e., $q_{1}=q_{5}$, $q_{4}=q_{8}, q_{1}^{\prime}=q_{5}^{\prime}, q_{4}^{\prime}=q_{8}^{\prime}$, or they merge, which contradicts the fact that $\left(\vec{q}^{\prime}, \vec{\varepsilon}\right)$ is not reachable by a synchronous execution; or the system is not GMC, which contradicts our assumptions.

\section{D.6 Complexity Analysis: Proofs of Propositions 3.1 and 3.2}

In this section, we fix $S=\left(M_{\mathrm{p}}\right)_{\mathrm{p} \in \mathcal{P}}, T S(S)=\left(N, n_{0}, \hat{E}, \Longrightarrow\right)$, and we let $\delta_{\mathrm{M}}$ be the biggest set of transitions of all the machines.

We first describe the maximum size of the basic constructions.

- $|N|=\prod_{\mathrm{p} \in \mathcal{P}}\left|Q_{\mathrm{p}}\right|$ since each node in $N$ consists of a $|\mathcal{P}|$ vector of states, each in $Q_{\mathrm{p}}$.

- $|\hat{\delta}|=|E|=\left.|2 \mathbb{A} \times| N\right|^{2} \mid$ since there can be at most $2 \mathbb{A}$ transitions between two nodes in $N$, indeed:

- the local state components of each event is a determined by the node it is fired from,

- by construction, given two nodes, all the transitions between these nodes must involve the same machines $s$ and $r$,

- the machines are deterministic, thus they can only send a same message once per state, and

- each message may be sent by each one of the two machines.

- $|T S(S)|=|N|+|\hat{\delta}|$

- $|\hat{E}|=|E|$, since, in the worst case, $\bowtie$ is the identity relation.

- Relation $<$ can be a computed via a breadth-first traversal of $T S(S)$, i.e., in $O(|N|+|\Longrightarrow|)$ time, and its maximal size if $|N|^{2}$. 
Note that it is not necessary to compute the transitive closure of $\prec$ since, to check whether a node is a "last node", it suffices to check its (direct) neighbours.

Proposition D.1. The $\bowtie$-relation is computable in time

$$
O\left(\sum_{\mathrm{p} \in \mathcal{P}}\left(\left|\delta_{\mathrm{p}}\right|^{6}\right)+\left(|E|^{2} \times\left(2 \times\left|\delta_{\mathrm{M}}\right|^{2}\right)\right)\right)
$$

Proof. First, we observe that computing the $\bullet$-relation for each machine can be done in time $\sum_{\mathrm{p} \in \mathcal{P}}\left(\left|\delta_{\mathrm{p}}\right|^{6}\right)$ since the size of the nontransitive version of $\bullet$ is $\left|\delta_{\mathrm{p}}^{2}\right|$ and computing the transitive closure of a binary relation has a cubic complexity. Then, to compute the $\bowtie$-relation, for each pair of events in $E$, we have to look up in two sets of $\bullet$ relations.

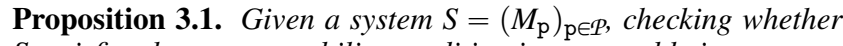
$S$ satisfies the representability condition is computable in

$$
O\left(\sum_{\mathrm{p} \in \mathcal{P}} 2^{|N|+\left|Q_{\mathrm{p}}\right|}\right) \text { time, with }|N|=\prod_{\mathrm{p} \in \mathcal{P}}\left|Q_{\mathrm{p}}\right|
$$

Proof. The first part of the definition requires, for each machine, to (i) compute its projection from $T S(S)$ (which depends on the size of $N$ ) and (ii) to check that this projection is language equivalent with the original machine. The second part requires for each machine (sum over $\mathcal{P}$ ), for each state $\left(Q_{\mathrm{p}}\right.$ factor), and for each transition from that state $\left(\delta_{\mathrm{p}}\right.$ factor) to look for a node in $T S(S)$ ( $N$ factor) such that this transition is reflected in $\operatorname{TS}(S)$ ( $\hat{\delta}$ factor). Since for each local state, we are only interested in the nodes where this local state appears, we divide by $\left|Q_{\mathrm{p}}\right|$. Hence, we have that the time complexity of the representability condition is:

$$
\begin{aligned}
& O\left(\sum_{\mathrm{p} \in \mathcal{P}}\left(|\hat{\delta}|+2^{|N|+\left|Q_{\mathrm{p}}\right|}\right)+\sum_{\mathrm{p} \in \mathcal{P}}\left(\left|Q_{\mathrm{p}} \times \delta_{\mathrm{p}} \times N\right| \times \frac{|\hat{\delta}|}{\left|Q_{\mathrm{p}}\right|}\right)\right) \\
= & O\left(\sum_{\mathrm{p} \in \mathcal{P}}\left(|\hat{\delta}|+2^{|N|+\left|Q_{\mathrm{p}}\right|}\right)+\sum_{\mathrm{p} \in \mathcal{P}}\left(\left|\delta_{\mathrm{p}} \times N \times \hat{\delta}\right|\right)\right) \\
= & O\left(\sum_{\mathrm{p} \in \mathcal{P}}\left(2^{|N|+\left|Q_{\mathrm{p}}\right|}\right)\right)
\end{aligned}
$$

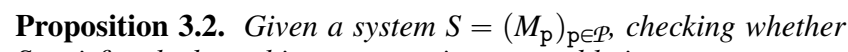
$S$ satisfies the branching property is computable in

$$
O\left(|\Longrightarrow|^{2} \times|\Longrightarrow| ! \times \sum_{\mathrm{r} \in \mathcal{P}}\left(\left|\delta_{\mathrm{r}}\right|^{2}\right)\right) \text { time. }
$$

Proof. The check of part (1) of Definition 3.5 is $O\left(|\Longrightarrow|^{2} \times|N|\right)$, so we consider the most complex case, namely part (2) of Definition 3.5. which is the sum of the complexity of conditions conditions 2a, 2b, and 2c of Definition 3.5 .

For each branch with events $e_{1} \neq e_{2}$ and each participant $\mathrm{p} \in \mathcal{P}$, we have to compute the sets $L_{\mathrm{p}}^{i}$. This also allows to compute the last nodes and can be done considering simple paths only, and is easily computed with a breadth-first visit of $T S(S)$, and therefore this is done in polynomial time in $|T S(S)|$.

Once this is done it is easy to see that the check of part 2b of Definition 3.5 is polynomial in $|\mathcal{P} \cup A c t|$.
The most complex part of the computation is to check conditions 2b) (ii) and 2c because it requires to check the paths in $T S(S)$ and the enumeration of all paths is computed in $O(|\Longrightarrow| !)$.

Finally, the check of condition $2 \mathrm{~b}$ (ii) can be done in

$$
O(2 \times|T S(S)|)
$$

while $2 \mathrm{2c}$ is checked in $O\left(|\Longrightarrow|^{2} \times|\Longrightarrow| ! \times \sum_{\mathrm{r} \in \mathcal{P}}\left(\left|\delta_{\mathrm{r}}\right|^{2}\right)\right)$ time.

\section{E. Proofs of Section 4}

\section{E.1 Equivalences}

Lemma B.1. If $T$ is the reachability graph of the Petri net $\mathbb{N}$ obtained from $T S(S)$ via the algorithm in [18], and $T^{\prime}$ is the reachability graph of the Petri net obtained after applying Transformation B.1, then $T \approx T^{\prime}$.

Proof. Trivial since $\mathbb{N}$ is safe and the only added transition is labelled by $\varepsilon$.

Lemma B.2. If $T$ (resp. $T^{\prime}$ ) is the reachability graph of the Petri net $\mathbb{N}$ obtained after Transformation B.1 (resp. Transformation B.2, then $T \approx T^{\prime}$.

Proof. Follows from the fact that $\mathbb{N}$ is safe and the only added transitions are labelled by $\varepsilon$.

Theorem 4.1 (Completeness). Given a GMC system $S=\left(M_{\mathrm{p}}\right)_{\mathrm{p} \in \mathcal{P} \text {, }}$ let $G$ be the global graph built from $S$ and let $T S(S)=\left(N, n_{0}, \hat{E}, \Longrightarrow\right.$ ). If $T S(S)$ is self-loop free (i.e. $\left.\forall n, n^{\prime} \in N: n \Longrightarrow n^{\prime} \Longrightarrow n \neq n^{\prime}\right)$,

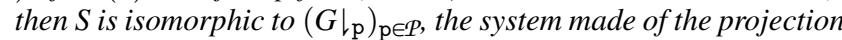
of $G$.

Proof. The proof follows from (1) the assumption that $T S(S)$ is self-loop free, (2) Lemmas B.1 and B.2, and (3) the fact that transformation to (pre-) global graph preserves the structure and labels of the joined net.

\section{E.2 Complexity Analysis of the Transformations}

Proposition B.1. Transformation B.1 is computable in linear time in the size of $m_{0}$.

Proof. Trivial.

Proposition B.2. Transformation B.2 is computable in polynomial time in the size of $\mathbb{N}$.

Proof. The algorithm for part (1) of the transformation works as follows: (1) compute the preset of each place $(|F|),(2)$ sort a table of pairs (preset, place) by preset, e.g., a lexicographic order on sets of transitions $(|P|(\log |P|))$, (3) go through the table and apply the transformation on each set of places which have the same preset $(|P|)$. Observe that, once a set of places with the same preset has been identified, the transformation can be done in linear time. The algorithm for part (2) works similarly.

Proposition B.3. Transformation B.3 is computable in polynomial time in the size of $\mathbb{N}$.

Proof. One has to iterate on each element in $P \cup T \cup F$ and the composition of graphs is polynomial in the size of the two graphs (number of vertices).

Proposition B.4. Transformation B.4 is computable in polynomial time in the size of $\mathbb{N}$. 
Proof. The transformation can be done by iterating on the arcs of the pre-global graph, so to find matching $\operatorname{arc}\left(|A|^{2}\right)$. 


\section{F. Appendix: Benchmark Examples from Section 5}

This section lists the most interesting synthesis examples selected from the benchmark given in Section 5 All benchmark examples are available in the online appendix ${ }^{3}$ and textual representations of these are also available in [2] (gmc-synthesis/tests/benchmark/gmc directory). Other GMC and non-GMC protocols are also available online ${ }^{4}$

\section{F.1 Alternating 3-bit protocol}

This protocol, adapted from [19], models a protocol where machine 0 repeatedly sends to machine 1 alternating messages $m_{l}, m_{2}$, and $m_{3}$ but will always concurrently wait for the acknowledgement $a_{i}$ before sending $m_{i}$.

3 http://www.doc.ic.ac.uk/ jlange/benchprotocols.pdf

4 http://www.doc.ic.ac.uk/ jlange/demo.tar.gz 


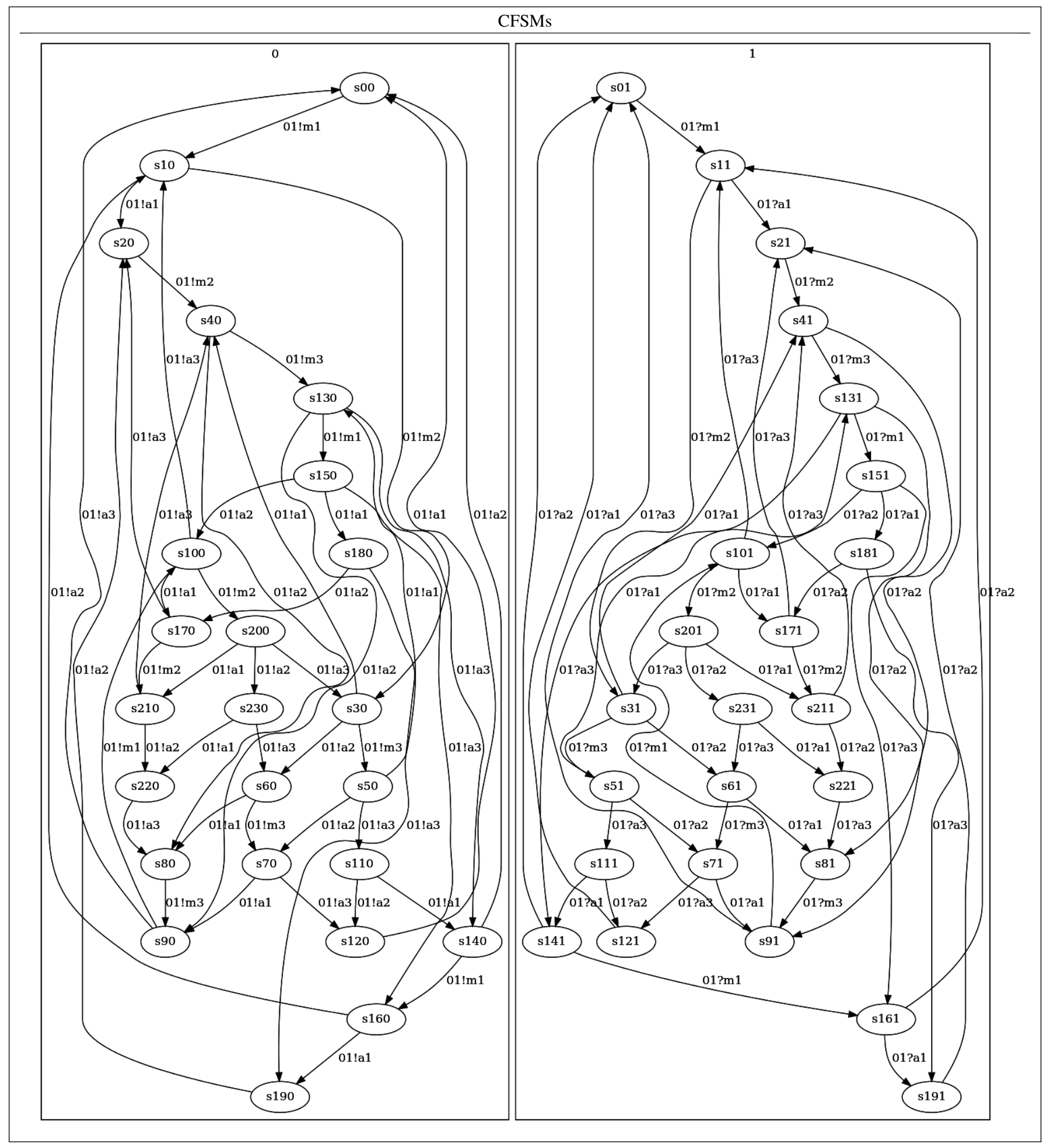




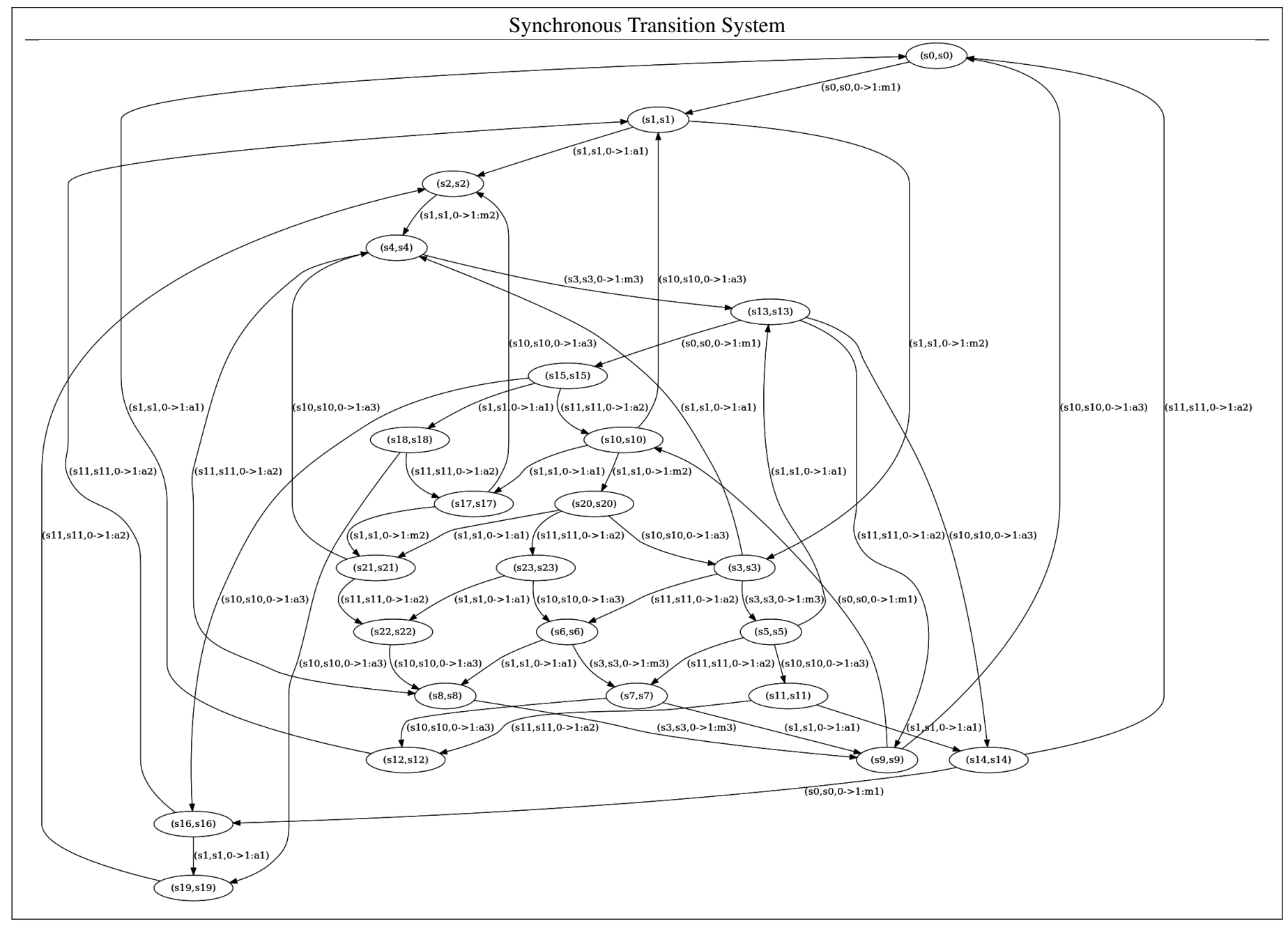




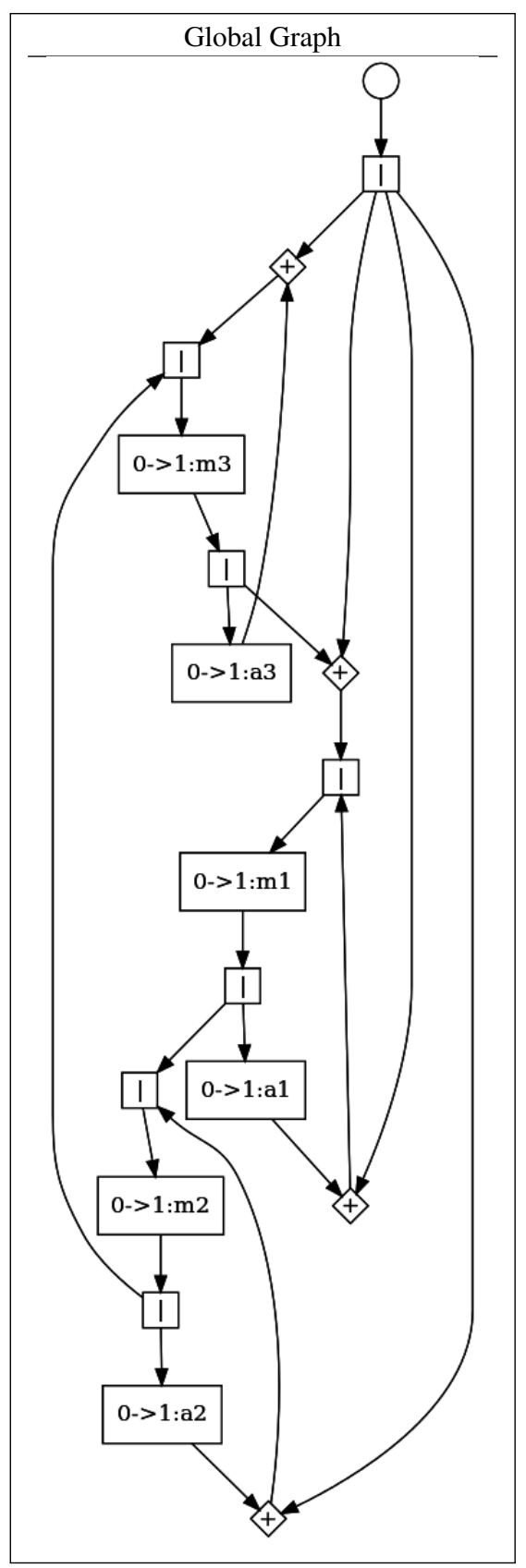




\section{F.2 Sanitary Agency $\times 2$}

This protocol, adapted from [30], models a software system that aims at "supporting elderly citizens in receiving sanitary assistance from the public administration". In this example, we consider the system consisting of the parallel composition of two instances of this protocol. In our formalisation, machine 0 is the Citizen, machine 1 is the Sanitary Agency, machine 2 is the Coop, and machine 3 is the Bank; and similarly for each machine $(4+i)(0 \leqslant i \leqslant 7)$.

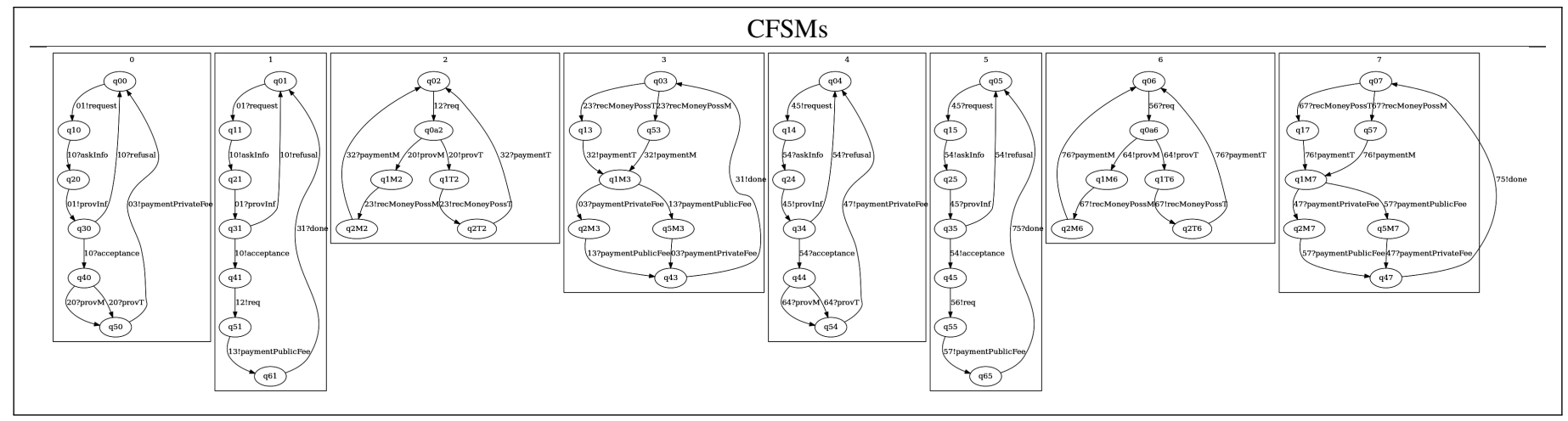




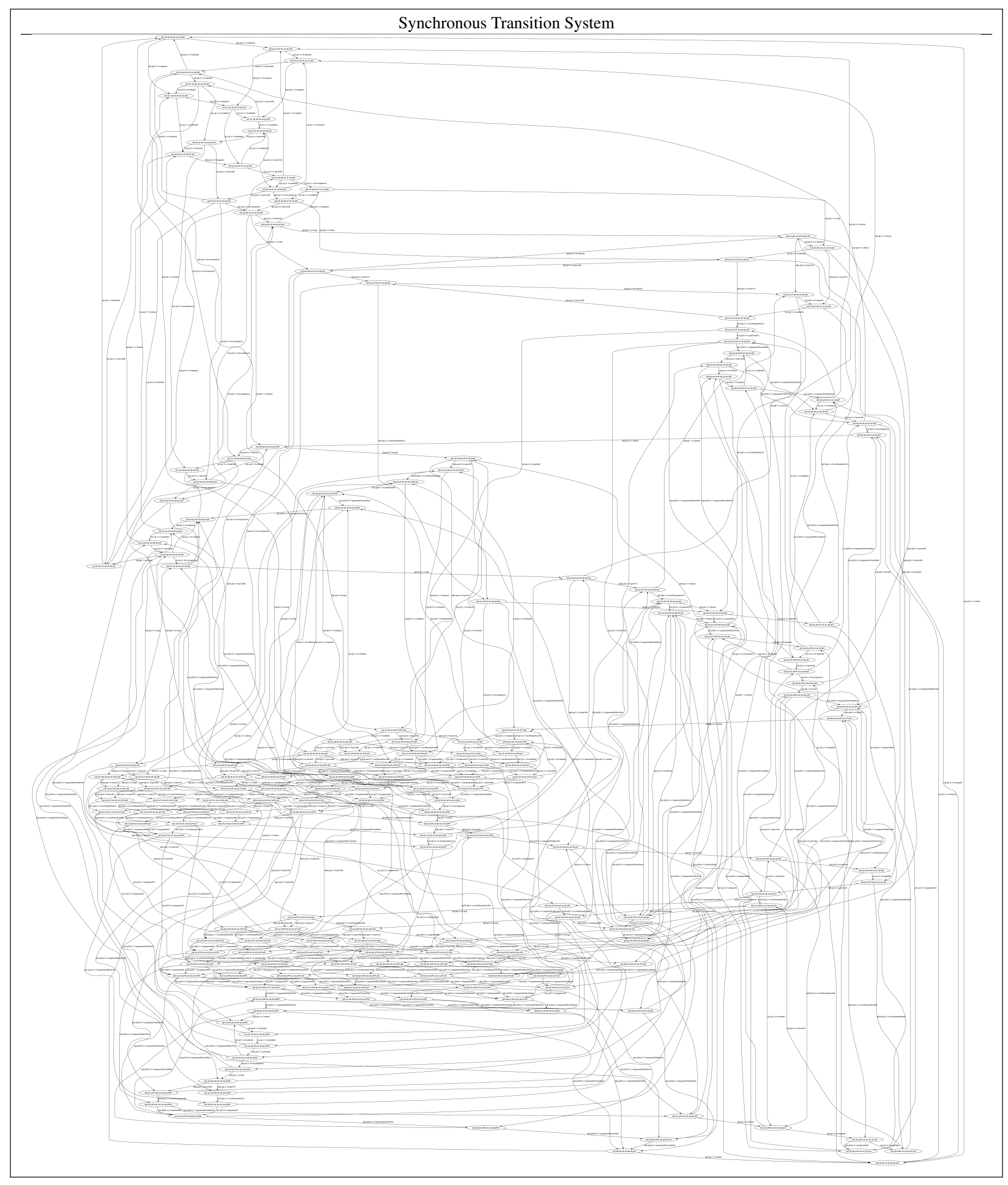




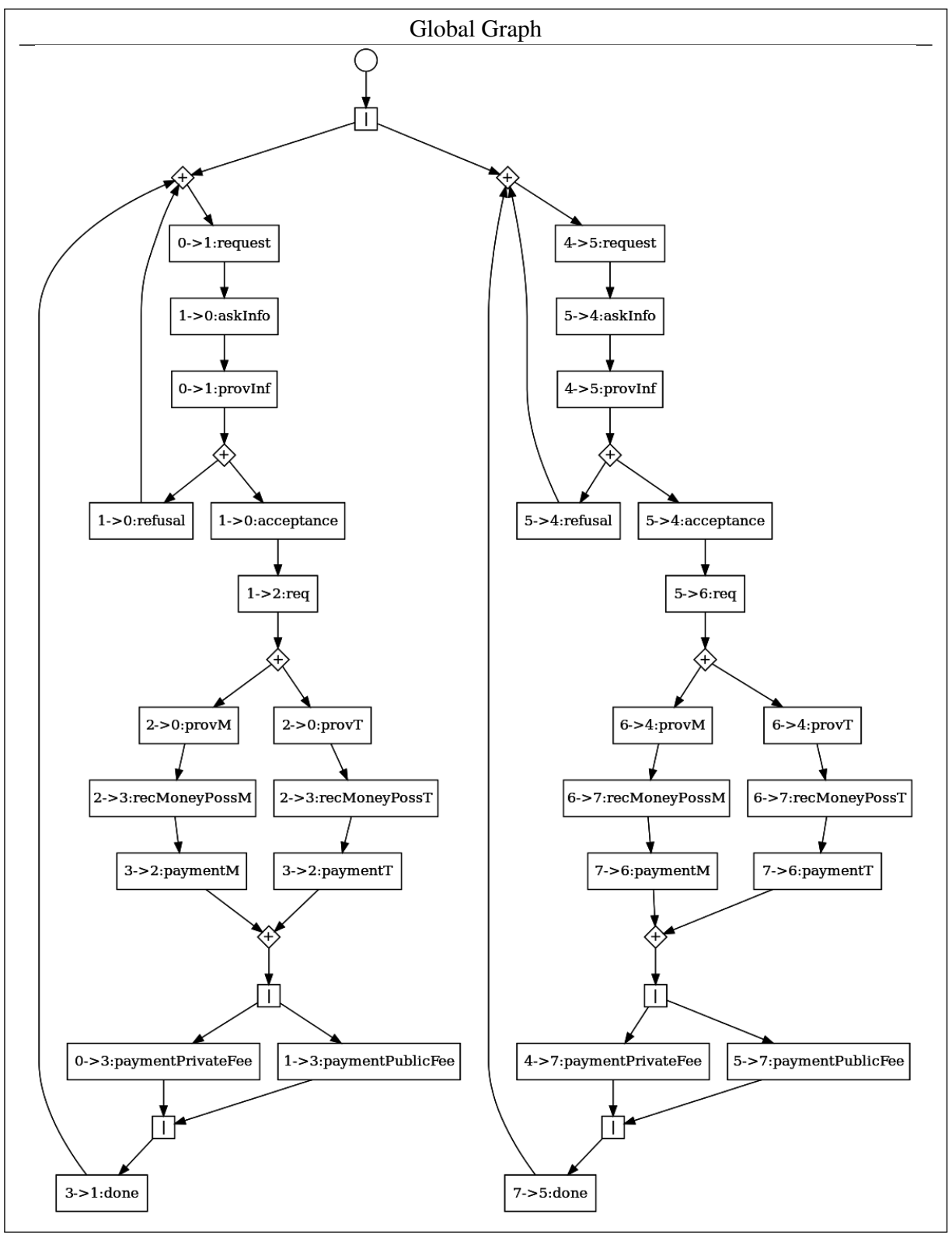

